\title{
On Singular Solutions to PDEs with Turning Point Involving a Quadratic Nonlinearity
}

\author{
Stéphane Malek \\ Laboratoire Paul Painlevé, University of Lille 1, 59655 Villeneuve d'Ascq Cedex, France \\ Correspondence should be addressed to Stéphane Malek; stephane.malek@math.univ-lille1.fr
}

Received 5 May 2017; Accepted 1 August 2017; Published 13 September 2017

Academic Editor: Sining Zheng

Copyright (C) 2017 Stéphane Malek. This is an open access article distributed under the Creative Commons Attribution License, which permits unrestricted use, distribution, and reproduction in any medium, provided the original work is properly cited.

\begin{abstract}
We study a singularly perturbed PDE with quadratic nonlinearity depending on a complex perturbation parameter $\epsilon$. The problem involves an irregular singularity in time, as in a recent work of the author and A. Lastra, but possesses also, as a new feature, a turning point at the origin in $\mathbb{C}$. We construct a family of sectorial meromorphic solutions obtained as a small perturbation in $\epsilon$ of a slow curve of the equation in some time scale. We show that the nonsingular parts of these solutions share common formal power series (that generally diverge) in $\epsilon$ as Gevrey asymptotic expansion of some order depending on data arising both from the turning point and from the irregular singular point of the main problem.
\end{abstract}

\section{Introduction}

In this work, we consider a family of nonlinear singularly perturbed equations of the form

$$
\begin{gathered}
Q\left(\partial_{z}\right)\left(P_{1}(t, \epsilon) u(t, z, \epsilon)+P_{2}(t, \epsilon) u^{2}(t, z, \epsilon)\right) \\
=f(t, z, \epsilon)+P_{3}\left(t, \epsilon, \partial_{t}, \partial_{z}\right) u(t, z, \epsilon),
\end{gathered}
$$

where $Q, P_{1}, P_{2}$, and $P_{3}$ are polynomials with complex coefficients and $f$ is an analytic function in the vicinity of the origin with respect to $t$ and $\epsilon$ in $\mathbb{C}$ and holomorphic with respect to $z$ on a horizontal strip in $\mathbb{C}$ of the form $H_{\beta}=\{z \in$ $\mathbb{C}|| \operatorname{Im}(z) \mid<\beta\}$ for some $\beta>0$.

Here we consider the case when $P_{1}(0, \epsilon)$ vanishes identically near 0 . The point $t=0$ is known to be called a turning point in that situation; see $[1,2]$ for a more detailed description of this terminology in the linear and nonlinear settings. Let us recall the definition of the valuation $\operatorname{val}_{t}(f)$ of an analytic function near $t=0$ as the smallest integer $k \geq 0$ with the factorization $f(t)=t^{k} \tilde{f}(t)$ for an analytic function $\tilde{f}$ near $t=0$ with $\widetilde{f}(0) \neq 0$. The most interesting case examined in this work is when the valuation $\operatorname{val}_{t}\left(P_{1}\right)$ of $P_{1}(t, \epsilon)$ with respect to $t$ is larger than the valuation $\operatorname{val}_{t}\left(P_{2}\right)$ or $\operatorname{val}_{t}(f(t, z, \epsilon))$ since the problem cannot be reduced to the case $P_{1}(0,0) \neq 0$ by dividing (1) by a suitable power of $t$ and $\epsilon$; see Remark 13 .

In our previous study [3], we already have considered a similar problem which corresponds to the situation when $P_{1}(0,0) \neq 0$ for our equation (1). Namely, we focused on the following problem:

$$
\begin{aligned}
Q\left(\partial_{z}\right) \partial_{t} y(t, z, \epsilon) \\
=\left(Q_{1}\left(\partial_{z}\right) y(t, z, \epsilon)\right)\left(Q_{2}\left(\partial_{z}\right) y(t, z, \epsilon)\right) \\
+H\left(t, \epsilon, \partial_{t}, \partial_{z}\right) y(t, z, \epsilon)+f(t, z, \epsilon)
\end{aligned}
$$

for given vanishing initial data $y(0, z, \epsilon) \equiv 0$, where $Q$, $Q_{1}, Q_{2}$, and $H$ are polynomials with complex coefficients and $f(t, z, \epsilon)$ is a forcing term constructed as above. Under appropriate assumptions on the shape of (2), we established the existence of a family of actual bounded holomorphic solutions $y_{p}(t, z, \epsilon), 0 \leq p \leq \varsigma-1$, for some integer $\varsigma \geq 2$, defined on domains $\mathscr{T} \times H_{\beta} \times \mathscr{E}_{p}$, for some fixed bounded sector $\mathscr{T}$ with vertex at 0 and $\mathscr{E}=\left\{\mathscr{E}_{p}\right\}_{0 \leq p \leq c-1}$, a set of bounded sectors whose union covers a full neighborhood of 0 in $\mathbb{C}^{*}$. These solutions are obtained by means of Laplace and inverse Fourier transforms. On each sector $\mathscr{E}_{p}$, they share with respect to $\epsilon$ a common asymptotic expansion $\hat{y}(t, z, \epsilon)=\sum_{n \geq 0} y_{n}(t, z) \epsilon^{n}$ which defines a formal series with 
bounded holomorphic coefficients on $\mathscr{T} \times H_{\beta}$. Moreover, this asymptotic expansion is shown to be of Gevrey order (at most) $1 / k$ that appears in the highest order term of the operator $H$ which is of irregular type in the sense of [4] outlined as $\epsilon^{\left(\delta_{D}-1\right) k} t^{\left(\delta_{D}-1\right)(k+1)} \partial_{t}^{\delta_{D}} R_{D}\left(\partial_{z}\right)$, for some integer $\delta_{D} \geq 2$ and a polynomial $R_{D}$ with complex coefficients. Conjointly, since the aperture of the sectors $\mathscr{E}_{p}$ can be chosen slightly larger than $\pi / k$, the functions $\epsilon \mapsto y_{p}(t, z, \epsilon)$ can be viewed as $k$-sums of the formal series $\hat{y}$ as defined in [5].

In this work, our goal is to achieve a similar statement, namely, the existence of sectorial holomorphic solutions and asymptotic expansions as $\epsilon$ tends to 0 . However, the main contrast with problem (2) is that, due to the presence of the turning point, our solutions are no longer bounded in the vicinity of the origin, being meromorphic in both time variable $t$ and parameter $\epsilon$. Namely, we build a set of actual meromorphic solutions $u^{\mathfrak{d}_{p}}(t, z, \epsilon)$ to problem (1) of the form

$$
u^{\mathfrak{D}_{p}}(t, z, \epsilon)=\epsilon^{\beta}\left(U_{0}\left(\epsilon^{\alpha} t\right)+\left(\epsilon^{\alpha} t\right)^{\gamma} v^{\mathfrak{D}_{p}}(t, z, \epsilon)\right),
$$

where $\alpha>1, \beta$ are some rational numbers, $\gamma$ is an integer, and $U_{0}(T)$ is a nonidentically vanishing root of a secondorder algebraic equation with polynomial coefficients related to the polynomials $P_{1}, P_{2}$, see (70), and where $v^{\mathfrak{b}_{p}}(t, z, \epsilon)$ is a bounded holomorphic function on products $\mathscr{T} \times H_{\beta} \times \mathscr{E}_{p}$ similar to the ones mentioned above, which can be expressed as a Laplace transform of some order $\kappa \geq 1$ and Fourier inverse transform

$$
\begin{gathered}
v^{\mathfrak{\delta}_{p}}(t, z, \epsilon)=\frac{\kappa}{(2 \pi)^{1 / 2}} \int_{-\infty}^{+\infty} \int_{L_{\mathfrak{b}_{p}}} \omega_{\kappa}^{\mathfrak{\delta}_{p}}(u, m, \epsilon) \\
\cdot \exp \left(-\left(\frac{u}{\epsilon^{\chi+\alpha} t}\right)^{\kappa}\right) e^{i z m} \frac{d u}{u} d m
\end{gathered}
$$

along some half line $L_{\mathfrak{d}_{p}}=\mathbb{R}_{+} e^{i \mathfrak{\delta}_{p}}$, for some positive rational number $\chi>0$, where $\omega_{\kappa}^{\mathfrak{d}_{p}}(u, m, \epsilon)$ represents a function with at most exponential growth of order $\kappa$ on a sector containing $L_{\mathfrak{b}_{p}}$ with respect to $u$, with exponential decay with respect to $m$ on $\mathbb{R}$ and with analytic dependence on $\epsilon$ near 0 (see Theorem 19). Furthermore, we show that these functions $v^{b_{p}}(t, z, \epsilon)$ own with respect to $\epsilon$ a common asymptotic expansion $\widehat{v}(t, z, \epsilon)=\sum_{n \geq 0} v_{n}(t, z) \epsilon^{n}$ which represents a formal series with bounded holomorphic coefficients on $\mathscr{T} \times$ $H_{\beta}$. We specify also the nature of this asymptotic expansion which turns out to be of Gevrey order (at most) $1 /(\chi+\alpha) \kappa$. Besides, since the aperture of the sectors $\mathscr{E}_{p}$ may be selected slightly larger than $\pi /(\chi+\alpha) \kappa$, the functions $v^{\delta_{p}}$ can be identified as $(\chi+\alpha) \kappa$-sums of the formal series $\widehat{v}$ (Theorem 21). By construction, the integer $\kappa$ shows up in the highest order term of the operator $P_{3}$ which is of irregular type of the form $\epsilon^{\Delta_{D}} t^{\delta_{D}(\kappa+1)+k_{0}} \partial_{t}^{\delta_{D}} R_{D}\left(\partial_{z}\right)$, with $k_{0}=\operatorname{val}_{t}\left(P_{1}\right)$, for some integers $\Delta_{D} \geq 0, \delta_{D} \geq 2$ and a polynomial $R_{D}$ with complex coefficients. The rational number $\chi$ is built with the help of the integers $\Delta_{D}, \delta_{D}, k_{0}$, and $\kappa$ and the rational numbers $\alpha, \beta$; see (86). According to the fact that $\alpha, \beta$ are mainly related to constraints assumed on the polynomials $P_{1}$ and $P_{2}$ (see (66), (67)), we observe that the Gevrey order $1 /(\chi+\alpha) \kappa$ of the asymptotic expansion involves information coming both from the highest irregular term and from the two polynomials $P_{1}$ and $P_{2}$ that shape the turning point at $t=0$, whereas, in our previous contribution [3], the Gevrey order was exclusively stemming from the irregular singularity at $t=0$.

The kind of equations with quadratic nonlinearity we investigate in this work is strongly related to singularly perturbed ODEs which are nonsingular at the origin of the form $\epsilon^{\sigma} d y / d t=F(t, y, a, \epsilon)$ for some analytic functions $F$, small complex parameter $\epsilon$, and a complex additional parameter $a$, described in the seminal joint paper by Canalis-Durand et al., see [6], where they study asymptotic properties of actual overstable solutions near a slow curve $\phi_{0}(t)$ (meaning that $\left.F\left(t, \phi_{0}(t), a, 0\right) \equiv 0\right)$ in the case when the Jacobian $\partial_{y} F\left(t, \phi_{0}(t), a, 0\right)$ is not invertible at $t=0$. The main notable difference is that we assume the origin to be at the same time a turning point and an irregular singularity. More precisely, with the rescaling map $(t, \epsilon) \mapsto(T=\epsilon t, \epsilon)$ the transformed equation (64) possesses a rational slow curve $U_{0}(T)$ and $T=0$ remains a turning point and an irregular singularity for this new equation.

The construction of the distinguished solution performed in Section 4 and the parametric Borel/Laplace summable character of these solutions shown in Section 7 are also intimately linked to recent developments of exact WKB analysis of formal and analytic solutions to second-order linear ODEs of Schrödinger type. Namely, let

$$
\epsilon^{2} \psi^{\prime \prime}(t, \epsilon)=Q(t) \psi(t, \epsilon)
$$

be a singularly perturbed ODE where $\epsilon$ is a small complex parameter and $Q(t)$ is some polynomial with complex coefficients. WKB solutions of (5) are known as special solutions that are described as an exponential $\widehat{\psi}(t, \epsilon)=$ $\exp \left(\int_{t_{0}}^{t} \widehat{S}(s, \epsilon) d s\right)$ where the expression $\widehat{S}(t, \epsilon)$ satisfies a socalled Riccati equation

$$
\epsilon^{2} \widehat{S}^{\prime}(t, \epsilon)+\epsilon^{2} \widehat{S}^{2}(t, \epsilon)=Q(t) .
$$

This last equation possesses formal power series solutions $\widehat{S}(t, \epsilon)=S_{-1}(t) / \epsilon+\sum_{n \geq 0} S_{n}(t) \epsilon^{n}$, where $S_{-1}(t)$ satisfies the quadratic equation $S_{-1}^{2}(t)=Q(t)$. Once $S_{-1}(t)= \pm \sqrt{Q(t)}$ is fixed, we get two formal solutions $\widehat{S}_{ \pm}(t, \epsilon)=S_{-1}(t) / \epsilon+\widehat{T}_{ \pm}(t, \epsilon)$, where $\widehat{T}_{ \pm}(t, \epsilon) \in \mathbb{C}[[\epsilon]]$ for any $t \in U=\{t \in \mathbb{C} \mid Q(t) \neq 0\}$. Notice that $\widehat{T}_{ \pm}(t, \epsilon)$ solves the first-order Riccati equation

$$
\epsilon \widehat{T}_{ \pm}^{\prime}(t, \epsilon)+2 S_{-1}(t) \widehat{T}_{ \pm}(t, \epsilon)+\epsilon \widehat{T}_{ \pm}^{2}(t, \epsilon)+S_{-1}^{\prime}(t)=0
$$

with turning points at the roots of $Q(t)$. Our main PDE (1) resembles this last one provided that $S_{-1}(t)$ is a polynomial and with the significant distinction that our equation only involves differential operators with irregular singularity at $t=0$. An essential feature of the theory is that the formal series $\widehat{T}_{ \pm}(t, \epsilon)$ are 1-summable in suitable directions $d \in \mathbb{R}$ with respect to $\epsilon$ (that are related to the function $\int_{t_{0}}^{t} S_{-1}(s) d s$ ) for any fixed $t \in U$. Different proofs of this fact can be found in [7-10]. Our second main statement, Theorem 21, can be considered as a similar contribution for some higher order 
PDEs of this latter result. Furthermore, in our study we are also able to describe the behaviour of our specific solutions near $(t, \epsilon)=(0,0)$.

For more recent and advanced works related to WKB analysis and local/global studies of solutions to linear ODEs near turning points, we refer to contributions related to the 1D Schrödinger equation with simple poles [11], with merging pairs of simple poles and turning points [12], and with merging triplet of poles and turning points [13, 14] and for analytic continuation properties of the Borel transform (resurgence) of WKB expansions in the problem of confluence of two simple turning points we quote [15]. Concerning the structure of singular formal solutions to singularly perturbed linear systems of ODEs with turning points we point out [16] solving an old question of Wasow. We mention also preeminent studies on WKB analysis for higher order differential equations which reveal new Stokes phenomena giving rise to so-called virtual turning points $[17,18]$.

In the framework of linear PDEs, normal forms for completely integrable systems near a degenerate point where two turning points coalesce have been obtained in [19], which is a first step toward the so-called Dubrovin conjecture which concerns the question of universal behaviour of generic solutions near gradient catastrophe of singularly Hamiltonian perturbations of first-order hyperbolic equations; see [20]. We mention also that sectorial analytic transformations to normal forms have been obtained for systems of singularly perturbed ODEs near a turning point with multiplicity using the recent approach of composite asymptotic expansions developed in [2]; see [21].

The paper is organized as follows. In Section 2, we recall the definition introduced in the work [3] of some weighted Banach spaces of continuous functions with exponential growth on unbounded sectors in $\mathbb{C}$ and with exponential decay on $\mathbb{R}$. We analyze the continuity of specific multiplication and linear/nonlinear convolution operators acting on these spaces.

In Section 3, we remind the reader of basic statements concerning $m_{k}$-Borel-Laplace transforms, a version of the classical Borel-Laplace maps already used in previous works $[3,22,23]$ and Fourier transforms acting on exponentially flat functions.

In Section 4, we display our main problems and explain the leading strategy in order to solve them. It consists in four operations. In a first step, we restrict our inquiry for the sets of solutions to time rescaled function spaces; see (63). Then, we consider candidates for solutions to the resulting auxiliary problem (64) that are small perturbations of a so-called slow curve which solves a second-order algebraic equation and which may be singular at the origin in $\mathbb{C}$. In a third step, we search again for time rescaled functions solutions for the associated problem (84) solved by the small perturbation of the slow curve; see (85). In the last step, we write down the convolution problem (95) solved by a suitable $m_{\kappa}$-Borel transform of a formal solution to the attached problem (87).

In Section 5, we solve the main convolution problem (95) within the Banach spaces described in Section 2 using some fixed point theorem argument.
In Section 6, we provide a set of actual meromorphic solutions to our initial equation (61) by executing backwards the operations described in Section 4. In particular, we show that our singular functions actually solve problem (164) which is a factorized part of (61) with a more restrictive forcing term. Furthermore, the difference of any two neighboring solutions tends to 0 as $\epsilon$ tends to 0 faster than a function with exponential decay of order $(\chi+\alpha) \kappa$.

In Section 7 , we show the existence of a common asymptotic expansion of Gevrey order $1 /(\chi+\alpha) \kappa$ for the nonsingular parts of these solutions of (61) and (164) based on the flatness estimates obtained in Section 6 using a theorem by Ramis and Sibuya.

\section{Banach Spaces with Exponential Growth and Exponential Decay}

We denote by $D(0, \rho)$ the open disc centered at 0 with radius $\rho>0$ in $\mathbb{C}$ and by $\bar{D}(0, \rho)$ its closure. Let $S_{d}$ be an open unbounded sector in direction $d \in \mathbb{R}$ and $\mathscr{E}$ be an open sector with finite radius $r_{\mathscr{E}}$, both centered at 0 in $\mathbb{C}$. By convention, these sectors do not contain the origin in $\mathbb{C}$. We first give definitions of Banach spaces which already appear in our previous work [3].

Definition 1. Let $\beta>0$ and $\mu>1$ be real numbers. We denote by $E_{(\beta, \mu)}$ the vector space of functions $h: \mathbb{R} \rightarrow \mathbb{C}$ such that

$$
\|h(m)\|_{(\beta, \mu)}=\sup _{m \in \mathbb{R}}(1+|m|)^{\mu} \exp (\beta|m|)|h(m)|
$$

is finite. The space $E_{(\beta, \mu)}$ endowed with the norm $\|\cdot\|_{(\beta, \mu)}$ becomes a Banach space.

As a direct consequence of Proposition 5 from [3], we notice the following.

Proposition 2. The Banach space $\left(E_{(\beta, \mu)},\|\cdot\|_{(\beta, \mu)}\right)$ is a Banach algebra for the convolution product

$$
(f \star g)(m)=\int_{-\infty}^{+\infty} f\left(m-m_{1}\right) g\left(m_{1}\right) d m_{1}
$$

Namely, there exists a constant $C_{0}>0$ (depending on $\mu$ ) such that

$$
\|(f \star g)(m)\|_{(\beta, \mu)} \leq C_{0}\|f(m)\|_{(\beta, \mu)}\|g(m)\|_{(\beta, \mu)}
$$

for all $f, g \in E_{(\beta, \mu)}$.

Definition 3. Let $\nu, \rho>0$ and $\beta>0, \mu>1$ be real numbers. Let $\kappa \geq 1$ and $\chi, \alpha \geq 0$ be integers. Let $\epsilon \in \mathscr{E}$. We denote by $F_{(\nu, \beta, \mu, \chi, \alpha, \kappa, \epsilon)}^{d}$ the vector space of continuous 
functions $(\tau, m) \mapsto h(\tau, m)$ on $\left(\bar{D}(0, \rho) \cup S_{d}\right) \times \mathbb{R}$, which are holomorphic with respect to $\tau$ on $D(0, \rho) \cup S_{d}$ and such that

$$
\begin{aligned}
& \|h(\tau, m)\|_{(\nu, \beta, \mu, \chi, \alpha, \kappa, \epsilon)} \\
& =\sup _{\tau \in \bar{D}(0, \rho) \cup S_{d}, m \in \mathbb{R}}(1+|m|)^{\mu} \exp (\beta|m|) \\
& \cdot \frac{1+\left|\tau / \epsilon^{\chi+\alpha}\right|^{2 \kappa}}{\left|\tau / \epsilon^{\chi+\alpha}\right|} \exp \left(-\nu\left|\frac{\tau}{\epsilon^{\chi+\alpha}}\right|^{\kappa}\right)|h(\tau, m)|
\end{aligned}
$$

is finite. One can check that the normed space $\left(F_{(\nu, \beta, \mu, \chi, \alpha, \kappa, \epsilon)}^{d}\right.$, $\left.\|\cdot\|_{(\nu, \beta, \mu, \chi, \alpha, \kappa, \epsilon)}\right)$ is a Banach space.

Throughout the whole section, we keep the notations of Definitions 1 and 3.

In the next lemma, we check that some parameter depending functions with polynomial growth with respect to the variable $\tau$ and exponential decay with respect to the variable $m$, which will appear later on in our study (Section 5), belong to the Banach spaces described above.

Lemma 4. Let $\gamma_{1} \geq 0, \gamma_{2} \geq 1$ be integers. Let $\widetilde{R}(X)$ be a polynomial that belongs to $\mathbb{C}[X]$ such that $\widetilde{R}(\mathrm{im}) \neq 0$ for all $m \in \mathbb{R}$. We take a function $\widetilde{B}(m)$ located in $E_{(\beta, \mu)}$ and we consider a continuous function $a_{\gamma_{1}, \kappa}(\tau, m)$ on $\left(\bar{D}(0, \rho) \cup S_{d}\right) \times \mathbb{R}$, holomorphic with respect to $\tau$ on $D(0, \rho) \cup S_{d}$, such that

$$
\left|a_{\gamma_{1}, \kappa}(\tau, m)\right| \leq \frac{1}{\left(1+|\tau|^{\kappa}\right)^{\gamma_{1}}|\widetilde{R}(i m)|}
$$

for all $\tau \in \bar{D}(0, \rho) \cup S_{d}$, all $m \in \mathbb{R}$.

Then, the function $\epsilon^{-\chi \gamma_{2}} \tau^{\gamma_{2}} \widetilde{B}(m) a_{\gamma_{1}, \kappa}(\tau, m)$ belongs to $F_{(\nu, \beta, \mu, \chi, \alpha, \kappa, \epsilon)}^{d}$. Moreover, there exists a constant $C_{1}>0$ (depending on $\kappa$ and $\gamma_{2}$ ) such that

$$
\begin{gathered}
\left\|\epsilon^{-\chi \gamma_{2}} \tau^{\gamma_{2}} \widetilde{B}(m) a_{\gamma_{1}, \kappa}(\tau, m)\right\|_{(\nu, \beta, \mu, \chi, \alpha, \kappa, \epsilon)} \\
\leq C_{1} \frac{\|\widetilde{B}(m)\|_{(\beta, \mu)}}{\inf _{m \in \mathbb{R}}|\widetilde{R}(i m)|}|\epsilon|^{\gamma_{2} \alpha}
\end{gathered}
$$

for all $\epsilon \in \mathscr{E}$.

Proof. By definition of the norm and bearing in mind the constraint on the polynomial $\widetilde{R}(X)$, we can write

$$
\begin{aligned}
& \left\|\epsilon^{-\chi \gamma_{2}} \tau^{\gamma_{2}} \widetilde{B}(m) a_{\gamma_{1}, \kappa}(\tau, m)\right\|_{(\nu, \beta, \mu, \chi, \alpha, \kappa, \epsilon)} \\
& \leq \sup _{\tau \in \bar{D}(0, \rho) \cup S_{d}, m \in \mathbb{R}}(1+|m|)^{\mu} \exp (\beta|m|)|\widetilde{B}(m)| \\
& \quad \times \frac{1+\left|\tau / \epsilon^{\chi+\alpha}\right|^{2 \kappa}}{\left|\tau / \epsilon^{\chi+\alpha}\right|} \exp \left(-\nu\left|\frac{\tau}{\epsilon^{\chi+\alpha}}\right|^{\kappa}\right)|\epsilon|^{-\chi \gamma_{2}}\left|\frac{\tau}{\epsilon^{\chi+\alpha}}\right|^{\gamma_{2}} \\
& \cdot|\epsilon|^{\gamma_{2}(\chi+\alpha)} \frac{1}{\left(1+\left|\tau / \epsilon \chi^{\chi+\alpha}\right|^{\kappa}|\epsilon|^{\kappa(\chi+\alpha)}\right)^{\gamma_{1}}|\widetilde{R}(i m)|} \\
& \leq \frac{\|\widetilde{B}(m)\|_{(\beta, \mu)}|\epsilon|^{\gamma_{2} \alpha}}{\inf _{m \in \mathbb{R}}|\widetilde{R}(i m)|}
\end{aligned}
$$

$$
\begin{aligned}
& \cdot \sup _{x \geq 0} \frac{1+x^{2 \kappa}}{x} x^{\gamma_{2}} \frac{e^{-\nu x^{\kappa}}}{\left(1+x^{\kappa}|\epsilon|^{\kappa(\chi+\alpha)}\right)^{\gamma_{1}}} \\
& \leq \frac{\|\widetilde{B}(m)\|_{(\beta, \mu)}|\epsilon|^{\gamma_{2} \alpha} \sup _{x \geq 0} \frac{1+x^{2 \kappa}}{x} x^{\gamma_{2}} e^{-\nu x^{\kappa}}}{\inf _{m \in \mathbb{R}}|\widetilde{R}(i m)|}
\end{aligned}
$$

which yields the lemma since an exponential grows faster than any polynomial.

The next proposition provides norm estimates for some linear convolution operators acting on the Banach spaces introduced above. These bounds are more accurate than the one supplied in Proposition 2 from [3]. These new estimates will be essential in Section 5 in order to solve problem (95). The improvements are due to the use of thorough upper bounds estimates of a generalized Mittag-Leffler function described in the proofs of Propositions 1 and 5 from [23].

Proposition 5. Let $\gamma_{j}, 0 \leq j \leq 3$, be real numbers with $\gamma_{1} \geq 0$. Let $\widetilde{R}(X)$ and $\widetilde{R}_{D}(X)$ be polynomials with complex coefficients such that $\operatorname{deg}(\widetilde{R}) \leq \operatorname{deg}\left(\widetilde{R}_{D}\right)$ and with $\widetilde{R}_{D}($ im $) \neq 0$ for all $m \in$ $\mathbb{R}$. We consider a continuous function $a_{\gamma_{1}, \kappa}(\tau, m)$ on $(\bar{D}(0, \rho) \cup$ $\left.S_{d}\right) \times \mathbb{R}$, holomorphic with respect to $\tau$ on $D(0, \rho) \cup S_{d}$, such that

$$
\left|a_{\gamma_{1}, \kappa}(\tau, m)\right| \leq \frac{1}{\left(1+|\tau|^{\kappa}\right)^{\gamma_{1}}\left|\widetilde{R}_{D}(i m)\right|}
$$

for all $\tau \in \bar{D}(0, \rho) \cup S_{d}$, all $m \in \mathbb{R}$. We make the following assumptions:

$$
\begin{aligned}
\frac{1}{\kappa}+\gamma_{3}+1 & >0, \\
\gamma_{2}+\gamma_{3}+2 & \geq 0, \\
\gamma_{2} & >-1 .
\end{aligned}
$$

(1) If $1+\gamma_{3} \leq 0$, then there exists a constant $C_{2}>0$ (depending on $v, \kappa, \gamma_{2}, \gamma_{3}$ and $\widetilde{R}(X), \widetilde{R}_{D}(X)$ ) such that

$$
\begin{aligned}
& \| \epsilon^{-\gamma_{0}} a_{\gamma_{1}, \kappa}(\tau, m) \widetilde{R}(i m) \\
& \cdot \tau^{\kappa} \int_{0}^{\tau^{\kappa}}\left(\tau^{\kappa}-s\right)^{\gamma_{2}} s^{\gamma_{3}} f\left(s^{1 / \kappa}, m\right) d s \|_{(\nu, \beta, \mu, \chi, \alpha, \kappa, \epsilon)} \\
& \quad \leq C_{2}|\epsilon|^{(\chi+\alpha) \kappa\left(\gamma_{2}+\gamma_{3}+2\right)-\gamma_{0}}\|f(\tau, m)\|_{(\nu, \beta, \mu, \chi, \alpha, \kappa, \epsilon)}
\end{aligned}
$$

for all $f(\tau, m) \in F_{(\nu, \beta, \mu, \chi, \alpha, \kappa, \epsilon)}^{d}$.

(2) If $1+\gamma_{3}>0$ and $\gamma_{1} \geq 1+\gamma_{3}$, then there exists a constant $C_{2}^{\prime}>0$ (depending on $\nu, \kappa, \gamma_{1}, \gamma_{2}, \gamma_{3}$ and $\widetilde{R}(X), \widetilde{R}_{D}(X)$ ) such that

$$
\| \epsilon^{-\gamma_{0}} a_{\gamma_{1}, \kappa}(\tau, m) \widetilde{R}(i m)
$$




$$
\begin{aligned}
& \cdot \tau^{\kappa} \int_{0}^{\tau^{\kappa}}\left(\tau^{\kappa}-s\right)^{\gamma_{2}} s^{\gamma_{3}} f\left(s^{1 / \kappa}, m\right) d s \|_{(\nu, \beta, \mu, \chi, \alpha, \kappa, \epsilon)} \\
& \leq C_{2}^{\prime}|\epsilon|^{(\chi+\alpha) \kappa\left(\gamma_{2}+\gamma_{3}+2\right)-\gamma_{0}-(\chi+\alpha) \kappa \gamma_{1}} \\
& \cdot\|f(\tau, m)\|_{(\nu, \beta, \mu, \chi, \alpha, \kappa, \epsilon)}
\end{aligned}
$$

for all $f(\tau, m) \in F_{(\nu, \beta, \mu, \chi, \alpha, \kappa, \epsilon)}^{d}$.

Proof. By definition of the norm, we can write

$$
\begin{aligned}
A & =\| \epsilon^{-\gamma_{0}} a_{\gamma_{1}, \kappa}(\tau, m) \widetilde{R}(i m) \tau^{\kappa} \int_{0}^{\tau^{\kappa}}\left(\tau^{\kappa}-s\right)^{\gamma_{2}} \\
& \cdot s^{\gamma_{3}} f\left(s^{1 / \kappa}, m\right) d s \|_{(\nu, \beta, \mu, \chi, \alpha, \kappa, \epsilon)}(1+|m|)^{\mu} \exp (\beta|m|) \\
& \leq \sup _{\tau \in \bar{D}(0, \rho) \cup S_{d}, m \in \mathbb{R}} \\
& \cdot \frac{1+\left|\tau / \epsilon^{\chi+\alpha}\right|^{2 \kappa}}{\left|\tau / \epsilon \epsilon^{+\alpha}\right|} \exp \left(-\nu\left|\frac{\tau}{\epsilon^{\chi+\alpha}}\right|^{\kappa}\right)|\epsilon|^{-\gamma_{0}} \frac{1}{\left(1+|\tau|^{\kappa}\right)^{\gamma_{1}}} \\
& \cdot \frac{|\widetilde{R}(i m)|}{\left|\widetilde{R}_{D}(i m)\right|} \times \mid \tau^{\kappa} \int_{0}^{\tau^{\kappa}}\left\{(1+|m|)^{\mu} \exp (\beta|m|)\right. \\
& \cdot \frac{1+|s|^{2} /|\epsilon|^{(\chi+\alpha) 2 \kappa}}{|s|^{1 / \kappa} /|\epsilon|^{\chi+\alpha}} \exp \left(-\nu \frac{|s|}{|\epsilon|^{(\chi+\alpha) \kappa}}\right) \\
& \left.\cdot f\left(s^{1 / \kappa}, m\right)\right\} \mathscr{A}(\tau, s, m, \epsilon) d s \mid,
\end{aligned}
$$

where

$$
\begin{aligned}
\mathscr{A}(\tau, s, m, \epsilon)= & \frac{1}{(1+|m|)^{\mu} \exp (\beta|m|)} \\
& \cdot \frac{\exp \left(\nu\left(|s| /|\epsilon|^{(\chi+\alpha) \kappa}\right)\right)}{\left(1+|s|^{2} /|\epsilon|^{(\chi+\alpha) 2 \kappa}\right)} \\
& \cdot \frac{|s|^{1 / \kappa}}{|\epsilon|^{\chi+\alpha}}\left(\tau^{\kappa}-s\right)^{\gamma_{2}} s^{\gamma_{3}} .
\end{aligned}
$$

Again by the definition of the norm of $f$ and by the constraints on the polynomials $R, R_{D}$, we deduce that

$$
A \leq C_{2.1}(\epsilon) \sup _{m \in \mathbb{R}}\left|\frac{\widetilde{R}(i m)}{\widetilde{R}_{D}(i m)}\right|\|f(\tau, m)\|_{(\nu, \beta, \mu, \chi, \alpha, \kappa, \epsilon)},
$$

where

$$
\begin{gathered}
C_{2.1}(\epsilon)=\sup _{\tau \in \bar{D}(0, \rho) \cup S_{d}} \frac{1+\left|\tau / \epsilon^{\chi+\alpha}\right|^{2 \kappa}}{\left|\tau / \epsilon^{\chi+\alpha}\right|} \exp \left(-\nu\left|\frac{\tau}{\epsilon \chi^{\chi \alpha}}\right|^{\kappa}\right) \\
\cdot|\epsilon|^{-\gamma_{0}} \frac{1}{\left(1+|\tau|^{\kappa}\right)^{\gamma_{1}}} \times|\tau|^{\kappa} \\
\cdot \int_{0}^{|\tau|^{\kappa}} \frac{\exp \left(\nu\left(h /|\epsilon|^{(\chi+\alpha) \kappa}\right)\right)}{1+h^{2} /|\epsilon|^{(\chi+\alpha) 2 \kappa}} \frac{h^{1 / \kappa}}{|\epsilon|^{\chi+\alpha}}\left(|\tau|^{\kappa}-h\right)^{\gamma_{2}} h^{\gamma_{3}} d h .
\end{gathered}
$$

We perform the change of variable $h=|\epsilon|^{(\chi+\alpha) \kappa} h^{\prime}$ inside the integral which is a part of $C_{2.1}(\epsilon)$ that yields

$$
\begin{aligned}
& C_{2.1}(\epsilon)=\sup _{\tau \in \bar{D}(0, \rho) \cup S_{d}} \frac{1+\left|\tau / \epsilon^{\chi+\alpha}\right|^{2 \kappa}}{\left|\tau / \epsilon^{\chi+\alpha}\right|} \exp \left(-\nu\left|\frac{\tau}{\epsilon^{\chi+\alpha}}\right|^{\kappa}\right) \\
& \cdot|\epsilon|^{-\gamma_{0}} \frac{1}{\left(1+|\tau|^{\kappa}\right)^{\gamma_{1}}} \times|\tau|^{\kappa} \\
& \cdot \int_{0}^{|\tau|^{\kappa} /|\epsilon|^{(\chi+\alpha) \kappa}} \frac{e^{\nu h^{\prime}}}{1+\left(h^{\prime}\right)^{2}}\left(h^{\prime}\right)^{1 / \kappa}\left(\frac{|\tau|^{\kappa}}{|\epsilon|^{(\chi+\alpha) \kappa}}-h^{\prime}\right)^{\gamma_{2}} \\
& \cdot\left(h^{\prime}\right)^{\gamma_{3}} d h^{\prime}|\epsilon|^{(\chi+\alpha) \kappa\left(\gamma_{2}+\gamma_{3}+1\right)} .
\end{aligned}
$$

As a result, we obtain the bounds

$$
\begin{aligned}
C_{2.1}(\epsilon) \leq & |\epsilon|^{(\chi+\alpha) \kappa\left(\gamma_{2}+\gamma_{3}+1\right)-\gamma_{0}+(\chi+\alpha) \kappa} \sup _{x \geq 0} \frac{1+x^{2}}{x^{1 / \kappa}} e^{-v x} \\
& \cdot \frac{x}{\left(1+|\epsilon|^{(\chi+\alpha) \kappa} x\right)^{\gamma_{1}}} G(x)
\end{aligned}
$$

where

$$
G(x)=\int_{0}^{x} \frac{e^{\nu h^{\prime}}}{1+\left(h^{\prime}\right)^{2}}\left(h^{\prime}\right)^{1 / \kappa+\gamma_{3}}\left(x-h^{\prime}\right)^{\gamma_{2}} d h^{\prime} .
$$

We now proceed as in Proposition 1 of [23]. We split the function $G(x)$ into two pieces and study them separately. Namely, we decompose $G(x)=G_{1}(x)+G_{2}(x)$, where

$$
\begin{aligned}
& G_{1}(x)=\int_{0}^{x / 2} \frac{e^{\nu h^{\prime}}}{1+\left(h^{\prime}\right)^{2}}\left(h^{\prime}\right)^{1 / \kappa+\gamma_{3}}\left(x-h^{\prime}\right)^{\gamma_{2}} d h^{\prime}, \\
& G_{2}(x)=\int_{x / 2}^{x} \frac{e^{\nu h^{\prime}}}{1+\left(h^{\prime}\right)^{2}}\left(h^{\prime}\right)^{1 / \kappa+\gamma_{3}}\left(x-h^{\prime}\right)^{\gamma_{2}} d h^{\prime} .
\end{aligned}
$$

We first provide estimates for $G_{1}(x)$.

(a) Assume that $-1<\gamma_{2}<0$. We see that $\left(x-h^{\prime}\right)^{\gamma_{2}} \leq$ $(x / 2)^{\gamma_{2}}$ for all $0 \leq h^{\prime} \leq x / 2$, for $x>0$. Hence, from the first constraint of (16), we get

$$
\begin{aligned}
G_{1}(x) & \leq\left(\frac{x}{2}\right)^{\gamma_{2}} e^{\nu x / 2} \int_{0}^{x / 2}\left(h^{\prime}\right)^{1 / \kappa+\gamma_{3}} d h^{\prime} \\
& =\left(\frac{x}{2}\right)^{\gamma_{2}} e^{\nu x / 2} \frac{(x / 2)^{1 / \kappa+\gamma_{3}+1}}{1 / \kappa+\gamma_{3}+1}
\end{aligned}
$$


for all $x \geq 0$. Subsequently, we obtain

$$
\begin{aligned}
& \sup _{x \geq 0} \frac{1+x^{2}}{x^{1 / \kappa}} e^{-v x} \frac{x}{\left(1+|\epsilon|^{(\chi+\alpha) \kappa} x\right)^{\gamma_{1}}} G_{1}(x) \\
& \quad \leq \sup _{x \geq 0} \frac{1+x^{2}}{x^{1 / \kappa}} e^{-v x} x G_{1}(x)
\end{aligned}
$$

which is finite due to the second assumption of (16).

(b) Assume that $\gamma_{2}>0$. We notice that $\left(x-h^{\prime}\right)^{\gamma_{2}} \leq x^{\gamma_{2}}$ for all $0 \leq h^{\prime} \leq x / 2$, for $x>0$. Therefore, again from the first constraint of (16) we get

$$
\begin{aligned}
G_{1}(x) & \leq x^{\gamma_{2}} e^{\nu x / 2} \int_{0}^{x / 2}\left(h^{\prime}\right)^{1 / \kappa+\gamma_{3}} d h^{\prime} \\
& =x^{\gamma_{2}} e^{\nu x / 2} \frac{(x / 2)^{1 / \kappa+\gamma_{3}+1}}{1 / \kappa+\gamma_{3}+1}
\end{aligned}
$$

for all $x \geq 0$. Consequently, we obtain

$$
\begin{aligned}
& \sup _{x \geq 0} \frac{1+x^{2}}{x^{1 / \kappa}} e^{-v x} \frac{x}{\left(1+|\epsilon|^{(\chi+\alpha) \kappa} x\right)^{\gamma_{1}}} G_{1}(x) \\
& \quad \leq \sup _{x \geq 0} \frac{1+x^{2}}{x^{1 / \kappa}} e^{-v x} x G_{1}(x)
\end{aligned}
$$

which is finite due to the second assumption of (16).

In a second step, we study $G_{2}(x)$.

We see that $1+\left(h^{\prime}\right)^{2} \geq 1+(x / 2)^{2}$ for all $x / 2 \leq h^{\prime} \leq x$. Hence,

$$
\begin{aligned}
G_{2}(x) & \leq \frac{1}{1+(x / 2)^{2}} \int_{x / 2}^{x} e^{\nu h^{\prime}}\left(h^{\prime}\right)^{1 / \kappa+\gamma_{3}}\left(x-h^{\prime}\right)^{\gamma_{2}} d h^{\prime} \\
& \leq \frac{1}{1+(x / 2)^{2}} G_{2.1}(x),
\end{aligned}
$$

where

$$
G_{2.1}(x)=\int_{0}^{x} e^{\nu h^{\prime}}\left(h^{\prime}\right)^{1 / \kappa+\gamma_{3}}\left(x-h^{\prime}\right)^{\gamma_{2}} d h^{\prime}
$$

for all $x \geq 0$. Taking account of the estimates (18) in [23] which are deduced from the asymptotic behaviour for large $x$ of the generalized Mittag-Leffler function $E_{\alpha, \beta}(x)=$ $\sum_{n \geq 0} x^{n} / \Gamma(\beta+n \alpha)$, for $\alpha, \beta>0$, we get a constant $K_{2.1}>0$ (that depends on $\nu, \kappa, \gamma_{2}, \gamma_{3}$ ) such that

$$
G_{2.1}(x) \leq K_{2.1} x^{1 / \kappa+\gamma_{3}} e^{\nu x}
$$

for all $x \geq 1$, provided the first and last constraints of (16) hold.
(1) We consider the first case when $1+\gamma_{3} \leq 0$. Bearing in mind (31) and (33), we deduce that

$$
\begin{aligned}
& \sup _{x \geq 1} \frac{1+x^{2}}{x^{1 / \kappa}} e^{-v x} \frac{x}{\left(1+|\epsilon|^{(\chi+\alpha) \kappa} x\right)^{\gamma_{1}}} G_{2}(x) \\
& \quad \leq \sup _{x \geq 1} \frac{1+x^{2}}{1+(x / 2)^{2}} K_{2.1} x^{1+\gamma_{3}}
\end{aligned}
$$

which is finite. On the other hand, when $0 \leq x<1$, we make the change of variable $h^{\prime}=x u^{\prime}$ inside $G_{2.1}(x)$ and, taking (31) into account, we get

$$
\begin{aligned}
\sup _{0 \leq x<1} & \frac{1+x^{2}}{x^{1 / \kappa}} e^{-v x} \frac{x}{\left(1+|\epsilon|^{(\chi+\alpha) \kappa} x\right)^{\gamma_{1}}} G_{2}(x) \\
\leq & \sup _{0 \leq x<1} \frac{1+x^{2}}{1+(x / 2)^{2}} e^{-v x} \frac{x}{x^{1 / \kappa}} x^{1 / \kappa+\gamma_{3}+\gamma_{2}+1} \\
& \times \int_{0}^{1} e^{\nu x u^{\prime}}\left(u^{\prime}\right)^{1 / \kappa+\gamma_{3}}\left(1-u^{\prime}\right)^{\gamma_{2}} d u^{\prime}
\end{aligned}
$$

which is finite provided that the constraints (16) are fulfilled.

(2) We examine the second case when $1+\gamma_{3}>0$ and $\gamma_{1} \geq \gamma_{3}+1$.

We use this time the fact that $1+|\epsilon|^{(\chi+\alpha) \kappa} x \geq|\epsilon|^{(\chi+\alpha) \kappa} x$ for all $x \geq 1$ and the bounds (33) in order to get

$$
\begin{gathered}
\sup _{x \geq 1} \frac{1+x^{2}}{x^{1 / \kappa}} e^{-\nu x} \frac{x}{\left(1+|\epsilon|^{(\chi+\alpha) \kappa} x\right)^{\gamma_{1}}} G_{2}(x) \\
\quad \leq|\epsilon|^{-(\chi+\alpha) \kappa \gamma_{1}} K_{2.1} \sup _{x \geq 1} \frac{1+x^{2}}{1+(x / 2)^{2}} \frac{x^{\gamma_{3}+1}}{x^{\gamma_{1}}} .
\end{gathered}
$$

On the other hand, the bounds on the domain $0 \leq x<1$ have already been treated above owing to (35).

Finally, gathering (21), (24), (28), (30), (34), (35), and (36) yields the statement of Proposition 5.

The forthcoming proposition presents norm estimates for some bilinear convolution operators acting on the aforementioned Banach spaces.

Proposition 6. There exists a constant $C_{3}>0$ (depending on $\mu$ and $\kappa)$ such that

$$
\begin{aligned}
& \| \tau^{\kappa-1} \int_{0}^{\tau^{\kappa}} \int_{-\infty}^{+\infty} f\left(\left(\tau^{\kappa}-s^{\prime}\right)^{1 / \kappa}, m-m_{1}\right) g\left(\left(s^{\prime}\right)^{1 / \kappa}, m_{1}\right) \\
& \cdot \frac{1}{\left(\tau^{\kappa}-s^{\prime}\right) s^{\prime}} d s^{\prime} d m_{1}\left\|_{(\nu, \beta, \mu, \chi, \alpha, \kappa, \epsilon)} \leq \frac{C_{3}}{|\epsilon|^{\chi+\alpha}}\right\| f(\tau, \\
& m)\left\|_{(\nu, \beta, \mu, \chi, \alpha, \kappa, \epsilon)}\right\| g(\tau, m) \|_{(\nu, \beta, \mu, \chi, \alpha, \kappa, \epsilon)} \\
& \text { for all } f(\tau), g(\tau) \in F_{(\nu, \beta, \mu, \chi, \alpha, \kappa, \epsilon)}^{d} .
\end{aligned}
$$

Proof. We follow the same guidelines as in the proof of Proposition 3 from [3]. By definition of the norm, we can write 


$$
\begin{aligned}
B= & \left\|\tau^{\kappa-1} \int_{0}^{\tau^{\kappa}} \int_{-\infty}^{+\infty} f\left(\left(\tau^{\kappa}-s^{\prime}\right)^{1 / \kappa}, m-m_{1}\right) g\left(\left(s^{\prime}\right)^{1 / \kappa}, m_{1}\right) \frac{1}{\left(\tau^{\kappa}-s^{\prime}\right) s^{\prime}} d s^{\prime} d m_{1}\right\|_{(\nu, \beta, \mu, \chi, \alpha, \kappa, \epsilon)}=\sup _{\tau \in \bar{D}(0, \rho) \cup s_{d}, m \in \mathbb{R}}(1+|m|)^{\mu} \exp (\beta|m|) \\
& \cdot \frac{1+\left|\tau / \epsilon^{\chi+\alpha}\right|^{2 \kappa}}{\left|\tau / \epsilon^{\chi+\alpha}\right|} \exp \left(-\nu\left|\frac{\tau}{\epsilon^{\chi+\alpha}}\right|^{\kappa}\right) \\
& \times \mid \tau^{\kappa-1} \int_{0}^{\tau^{\kappa}} \int_{-\infty}^{+\infty}\left\{\left(1+\left|m-m_{1}\right|\right)^{\mu} \exp \left(\beta\left|m-m_{1}\right|\right) \times \frac{1+\left|\tau^{\kappa}-s^{\prime}\right|^{2} /|\epsilon|^{(\chi+\alpha) 2 \kappa}}{\left|\tau^{\kappa}-s^{\prime}\right|^{1 / \kappa} /|\epsilon|^{\chi+\alpha}} \exp \left(-\nu \frac{\left|\tau^{\kappa}-s^{\prime}\right|}{|\epsilon|^{(\chi+\alpha) \kappa}}\right) f\left(\left(\tau^{\kappa}-s^{\prime}\right)^{1 / \kappa}, m-m_{1}\right)\right\} \\
& \times\left\{\left(1+\left|m_{1}\right|\right)^{\mu} \exp \left(\beta\left|m_{1}\right|\right) \frac{1+\left|s^{\prime}\right|^{2} /|\epsilon|^{(\chi+\alpha) 2 \kappa}}{\left|s^{\prime}\right|^{1 / \kappa} /|\epsilon|^{\chi+\alpha}} \exp \left(-v \frac{\left|s^{\prime}\right|}{|\epsilon|^{(\chi+\alpha) \kappa}}\right) g\left(\left(s^{\prime}\right)^{1 / \kappa}, m_{1}\right)\right\} \times \mathscr{B}\left(\tau, s, m, m_{1}\right) d s^{\prime} d m_{1} \mid,
\end{aligned}
$$

where

$$
\begin{aligned}
& \mathscr{B}\left(\tau, s, m, m_{1}\right)=\frac{\exp \left(-\beta\left|m-m_{1}\right|\right) \exp \left(-\beta\left|m_{1}\right|\right)}{\left(1+\left|m-m_{1}\right|\right)^{\mu}\left(1+\left|m_{1}\right|\right)^{\mu}} \\
& \cdot \frac{\left|s^{\prime}\right|^{1 / \kappa}\left|\tau^{\kappa}-s^{\prime}\right|^{1 / \kappa} /|\epsilon|^{2(\chi+\alpha)}}{\left(1+\left|\tau^{\kappa}-s^{\prime}\right|^{2} /|\epsilon|^{(\chi+\alpha) 2 \kappa}\right)\left(1+\left|s^{\prime}\right|^{2} /|\epsilon|^{(\chi+\alpha) 2 \kappa}\right)} \\
& \quad \times \exp \left(\nu \frac{\left|\tau^{\kappa}-s^{\prime}\right|}{|\epsilon|^{(\chi+\alpha) \kappa}}\right) \exp \left(v \frac{\left|s^{\prime}\right|}{|\epsilon|^{(\chi+\alpha) \kappa}}\right) \frac{1}{\left(\tau^{\kappa}-s^{\prime}\right) s^{\prime}} .
\end{aligned}
$$

By definition of the norms of $f$ and $g$ and according to the triangular inequality $|m| \leq\left|m-m_{1}\right|+\left|m_{1}\right|$ for all $m, m_{1} \in \mathbb{R}$, we deduce that

$B$

$$
\leq C_{3}(\epsilon)\|f(\tau, m)\|_{(\nu, \beta, \mu, \chi, \alpha, \kappa, \epsilon)}\|g(\tau, m)\|_{(\nu, \beta, \mu, \chi, \alpha, \kappa, \epsilon)},
$$

where

$$
\begin{aligned}
& C_{3}(\epsilon)=\sup _{\tau \in \bar{D}(0, \rho) \cup S_{d}, m \in \mathbb{R}}(1+|m|)^{\mu} \frac{1+\left|\tau / \epsilon^{\chi+\alpha}\right|^{2 \kappa}}{\left|\tau / \epsilon^{\chi+\alpha}\right|} \exp \left(-\nu\left|\frac{\tau}{\epsilon^{\chi+\alpha}}\right|^{\kappa}\right)|\tau|^{\kappa-1} \\
& \quad \times \int_{0}^{|\tau|^{\kappa}} \int_{-\infty}^{+\infty} \frac{1}{\left(1+\left|m-m_{1}\right|\right)^{\mu}\left(1+\left|m_{1}\right|\right)^{\mu}} \frac{\left(h^{\prime}\right)^{1 / \kappa}\left(|\tau|^{\kappa}-h^{\prime}\right)^{1 / \kappa}}{|\epsilon|^{2(\chi+\alpha)}} \frac{1}{\left(1+\left(|\tau|^{\kappa}-h^{\prime}\right)^{2} /|\epsilon|^{(\chi+\alpha) 2 \kappa}\right)\left(1+\left(h^{\prime}\right)^{2} /|\epsilon|^{(\chi+\alpha) 2 \kappa}\right)} \\
& \quad \times \exp \left(\nu \frac{|\tau|^{\kappa}-h^{\prime}}{|\epsilon|^{(\chi+\alpha) \kappa}}\right) \exp \left(\nu \frac{h^{\prime}}{|\epsilon|^{(\chi+\alpha) \kappa}}\right) \frac{1}{\left(|\tau|^{\kappa}-h^{\prime}\right) h^{\prime}} d h^{\prime} d m_{1} .
\end{aligned}
$$

We provide upper bounds that can be split in two parts,

$$
C_{3}(\epsilon) \leq C_{3.1} C_{3.2}(\epsilon),
$$

$$
\cdot \int_{-\infty}^{+\infty} \frac{1}{\left(1+\left|m-m_{1}\right|\right)^{\mu}\left(1+\left|m_{1}\right|\right)^{\mu}} d m_{1}
$$

where

$$
C_{3.1}=\sup _{m \in \mathbb{R}}(1+|m|)^{\mu}
$$

$$
C_{3.2}(\epsilon)=\sup _{\tau \in \bar{D}(0, \rho) \cup S_{d}} \frac{1+\left|\tau / \epsilon^{\chi+\alpha}\right|^{2 \kappa}}{\left|\tau / \epsilon^{\chi+\alpha}\right|}|\tau|^{\kappa-1} \times \int_{0}^{|\tau|^{\kappa}} \frac{\left(h^{\prime}\right)^{1 / \kappa}\left(|\tau|^{\kappa}-h^{\prime}\right)^{1 / \kappa} /|\epsilon|^{2(\chi+\alpha)}}{\left(1+\left(|\tau|^{\kappa}-h^{\prime}\right)^{2} /|\epsilon|^{(\chi+\alpha) 2 \kappa}\right)\left(1+\left(h^{\prime}\right)^{2} /|\epsilon|^{(\chi+\alpha) 2 \kappa}\right)} \frac{1}{\left(|\tau|^{\kappa}-h^{\prime}\right) h^{\prime}} d h^{\prime} .
$$


We carry out the change of variable $h^{\prime}=|\epsilon|^{(\chi+\alpha) \kappa} h$ inside the integral piece of $C_{3.2}(\epsilon)$ which yields the bounds

$$
\begin{aligned}
C_{3.2}(\epsilon)= & \sup _{\tau \in \bar{D}(0, \rho) \cup S_{d}} \frac{1+\left|\tau / \epsilon^{\chi+\alpha}\right|^{2 \kappa}}{\left|\tau / \epsilon^{\chi+\alpha}\right|}|\tau|^{\kappa-1} \\
& \times \int_{0}^{|\tau|^{\kappa} /|\epsilon|^{(\chi+\alpha) \kappa}} \frac{h^{1 / \kappa}\left(|\tau|^{\kappa} /|\epsilon|^{(\chi+\alpha) \kappa}-h\right)^{1 / \kappa}}{\left(1+\left(|\tau|^{\kappa} /|\epsilon|^{(\chi+\alpha) \kappa}-h\right)^{2}\right)\left(1+h^{2}\right)} \frac{1}{\left(|\tau|^{\kappa} /|\epsilon|^{(\chi+\alpha) \kappa}-h\right) h} \frac{1}{|\epsilon|^{(\chi+\alpha) \kappa}} d h \leq \frac{1}{|\epsilon|^{\chi+\alpha}} \sup _{x \geq 0} B(x),
\end{aligned}
$$

where

$$
\begin{aligned}
& B(x) \\
& \quad=\frac{1+x^{2}}{x^{2 / \kappa}} x \int_{0}^{x} \frac{h^{1 / \kappa}(x-h)^{1 / \kappa}}{\left(1+(x-h)^{2}\right)\left(1+h^{2}\right)} \frac{1}{(x-h) h} d h .
\end{aligned}
$$

A change of variable $h=x u$ in this last expression followed by a partial fraction decomposition allow us to write

$$
\begin{aligned}
& B(x)=\left(1+x^{2}\right) \\
& \cdot \int_{0}^{1} \frac{1}{\left(1+x^{2}(1-u)^{2}\right)\left(1+x^{2} u^{2}\right)} \frac{1}{(1-u)^{1-1 / \kappa} u^{1-1 / \kappa}} d u \\
& =\frac{1+x^{2}}{x^{2}+4} \int_{0}^{1} \frac{3-2 u}{1+x^{2}(1-u)^{2}} \frac{1}{(1-u)^{1-1 / \kappa} u^{1-1 / \kappa}} d u \\
& +\frac{1+x^{2}}{x^{2}+4} \int_{0}^{1} \frac{2 u+1}{1+x^{2} u^{2}} \frac{1}{(1-u)^{1-1 / \kappa} u^{1-1 / \kappa}} d u
\end{aligned}
$$

which acquaints us with the fact that $B(x)$ is finite provided that $\kappa \geq 1$ and bounded on $\mathbb{R}_{+}$with respect to $x$.

At last, collecting (38), (40), (42), (43), (45), and (47) leads to the statement of Proposition 6.

\section{Borel-Laplace and Fourier Transforms}

In this section, we review some basic statements concerning a $k$-Borel summability method of formal power series which is a slightly modified version of the more classical procedure (see [5], Section 3.2). This novel version has already been used in works such as $[3,22]$ when studying Cauchy problems under the presence of a small perturbation parameter. We remind also the reader of the definition of Fourier inverse transform acting on functions with exponential decay.

Definition 7. Let $k \geq 1$ be an integer. Let $\left(m_{k}(n)\right)_{n \geq 1}$ be the sequence

$$
m_{k}(n)=\Gamma\left(\frac{n}{k}\right)=\int_{0}^{\infty} t^{n / k-1} e^{-t} d t, \quad n \geq 1 .
$$

Let $\left(\mathbb{E},\|\cdot\|_{\mathbb{E}}\right)$ be a complex Banach space. We say a formal power series

$$
\widehat{X}(T)=\sum_{n=1}^{\infty} a_{n} T^{n} \in T \mathbb{E}[[T]]
$$

is $m_{k}$-summable with respect to $T$ in the direction $d \in[0,2 \pi)$ if the following assertions hold:

(1) There exists $\rho>0$ such that the $m_{k}$-Borel transform of $\widehat{X}, \mathscr{B}_{m_{k}}(\widehat{X})$, is absolutely convergent for $|\tau|<\rho$, where

$$
\mathscr{B}_{m_{k}}(\widehat{X})(\tau)=\sum_{n=1}^{\infty} \frac{a_{n}}{\Gamma(n / k)} \tau^{n} \in \tau \mathbb{E}[[\tau]] .
$$

(2) The series $\mathscr{B}_{m_{k}}(\widehat{X})$ can be analytically continued in a sector $S=\left\{\tau \in \mathbb{C}^{*}:|d-\arg (\tau)|<\delta\right\}$ for some $\delta>$ 0 . In addition to this, the extension is of exponential growth at most $k$ in $S$, meaning that there exist $C, K>$ 0 such that

$$
\left\|\mathscr{B}_{m_{k}}(\widehat{X})(\tau)\right\|_{\mathbb{E}} \leq C e^{K|\tau|^{k}}, \quad \tau \in S
$$

Under these assumptions, the vector valued Laplace transform of $\mathscr{B}_{m_{k}}(\widehat{X})$ along direction $d$ is defined by

$$
\begin{aligned}
& \mathscr{L}_{m_{k}}^{d}\left(\mathscr{B}_{m_{k}}(\widehat{X})\right)(T) \\
& =k \int_{L_{\gamma}} \mathscr{B}_{m_{k}}(\widehat{X})(u) e^{-(u / T)^{k}} \frac{d u}{u},
\end{aligned}
$$

where $L_{\gamma}$ is the path parametrized by $u \in[0, \infty) \mapsto u e^{i \gamma}$, for some appropriate direction $\gamma$ depending on $T$, such that $L_{\gamma} \subseteq S$ and $\cos (k(\gamma-\arg (T))) \geq \Delta>0$ for some $\Delta>0$.

The function $\mathscr{L}_{m_{k}}^{d}\left(\mathscr{B}_{m_{k}}(\widehat{X})\right)$ is well defined and turns out to be a holomorphic and bounded function in any sector of the form $S_{d, \theta, R^{1 / k}}=\left\{T \in \mathbb{C}^{\star}:|T|<R^{1 / k},|d-\arg (T)|<\theta / 2\right\}$, for some $\pi / k<\theta<\pi / k+2 \delta$ and $0<R<\Delta / K$. This function is known as the $m_{k}$-sum of the formal power series $\widehat{X}(T)$ in the direction $d$.

The following are some elementary properties concerning the $m_{k}$-sums of formal power series which will be crucial in our procedure.

(1) The function $\mathscr{L}_{m_{k}}^{d}\left(\mathscr{B}_{m_{k}}(\widehat{X})\right)(T)$ admits $\widehat{X}(T)$ as its Gevrey asymptotic expansion of order $1 / k$ with respect to $T$ 
in $S_{d, \theta, R^{1 / k}}$. More precisely, for every $\pi / k<\theta_{1}<\theta$, there exist $C, M>0$ such that

$$
\begin{aligned}
& \left\|\mathscr{L}_{m_{k}}^{d}\left(\mathscr{B}_{m_{k}}(\widehat{X})\right)(T)-\sum_{p=1}^{n-1} a_{p} T^{p}\right\|_{\mathbb{E}} \\
& \leq C M^{n} \Gamma\left(1+\frac{n}{k}\right)|T|^{n},
\end{aligned}
$$

for every $n \geq 2$ and $T \in S_{d, \theta_{1}, R^{1 / k} \text {. Watson's lemma }}$ (see Proposition 11 p. 75 in [25]) allows us to affirm that $\mathscr{L}_{m_{k}}^{d}\left(\mathscr{B}_{m_{k}}(\widehat{X})\right)(T)$ is unique provided that the opening $\theta_{1}$ is larger than $\pi / k$.

(2) Whenever $\mathbb{E}$ is a Banach algebra, the set of holomorphic functions having Gevrey asymptotic expansion of order $1 / k$ on a sector with values in $\mathbb{E}$ turns out to be a differential algebra (see Theorems 18, 19, and 20 in [25]). This and the uniqueness provided by Watson's lemma allow us to obtain some properties on $m_{k}$-summable formal power series in direction $d$.

By $\star$ we denote the product in the Banach algebra and also the Cauchy product of formal power series with coefficients in $\mathbb{E}$. Let $\widehat{X}_{1}, \widehat{X}_{2} \in T \mathbb{E}[[T]]$ be $m_{k}$-summable formal power series in direction $d$. Let $q_{1} \geq q_{2} \geq 1$ be integers. Then $\widehat{X}_{1}+\widehat{X}_{2}$, $\widehat{X}_{1} \star \widehat{X}_{2}$, and $T^{q_{1}} \partial_{T}^{q_{2}} \widehat{X}_{1}$, which are elements of $T \mathbb{E}[[T]]$, are $m_{k}$-summable in direction $d$. Moreover, one has

$$
\begin{aligned}
& \mathscr{L}_{m_{k}}^{d}\left(\mathscr{B}_{m_{k}}\left(\widehat{X}_{1}\right)\right)(T)+\mathscr{L}_{m_{k}}^{d}\left(\mathscr{B}_{m_{k}}\left(\widehat{X}_{2}\right)\right)(T) \\
& \quad=\mathscr{L}_{m_{k}}^{d}\left(\mathscr{B}_{m_{k}}\left(\widehat{X}_{1}+\widehat{X}_{2}\right)\right)(T), \\
& \mathscr{L}_{m_{k}}^{d}\left(\mathscr{B}_{m_{k}}\left(\widehat{X}_{1}\right)\right)(T) \star \mathscr{L}_{m_{k}}^{d}\left(\mathscr{B}_{m_{k}}\left(\widehat{X}_{2}\right)\right)(T) \\
& \quad=\mathscr{L}_{m_{k}}^{d}\left(\mathscr{B}_{m_{k}}\left(\widehat{X}_{1} \star \widehat{X}_{2}\right)\right)(T), \\
& T^{q_{1}} \partial_{T}^{q_{2}} \mathscr{L}_{m_{k}}^{d}\left(\mathscr{B}_{m_{k}}\left(\widehat{X}_{1}\right)\right)(T) \\
& \quad=\mathscr{L}_{m_{k}}^{d}\left(\mathscr{B}_{m_{k}}\left(T^{q_{1}} \partial_{T}^{q_{2}} \widehat{X}_{1}\right)\right)(T),
\end{aligned}
$$

for every $T \in S_{d, \theta, R^{1 / k}}$.

The next proposition is written without proof for it can be found in [3], Proposition 6.

Proposition 8. Let $\widehat{f}(t)=\sum_{n \geq 1} f_{n} t^{n}$ and $\widehat{g}(t)=\sum_{n \geq 1} g_{n} t^{n}$ which belong to $\mathbb{E}[[t]]$, where $\left(\mathbb{E},\|\cdot\|_{\mathbb{E}}\right)$ is a Banach algebra. Let $k, m \geq 1$ be integers. The following formal identities hold.

$$
\begin{gathered}
\mathscr{B}_{m_{k}}\left(t^{k+1} \partial_{t} \hat{f}(t)\right)(\tau)=k \tau^{k} \mathscr{B}_{m_{k}}(\hat{f}(t))(\tau), \\
\mathscr{B}_{m_{k}}\left(t^{m} \widehat{f}(t)\right)(\tau)=\frac{\tau^{k}}{\Gamma(m / k)} \\
\cdot \int_{0}^{\tau^{k}}\left(\tau^{k}-s\right)^{m / k-1} \mathscr{B}_{m_{k}}(\widehat{f}(t))\left(s^{1 / k}\right) \frac{d s}{s},
\end{gathered}
$$

$$
\begin{aligned}
\mathscr{B}_{m_{k}} & (\widehat{f}(t) \star \widehat{g}(t))(\tau) \\
= & \tau^{k} \int_{0}^{\tau^{k}} \mathscr{B}_{m_{k}}(\widehat{f}(t))\left(\left(\tau^{k}-s\right)^{1 / k}\right) \\
\star & \mathscr{B}_{m_{k}}(\widehat{g}(t))\left(s^{1 / k}\right) \frac{1}{\left(\tau^{k}-s\right) s} d s .
\end{aligned}
$$

In the last part of the section, we recall without proofs some properties of the inverse Fourier transform acting on continuous functions with exponential decay on $\mathbb{R}$; see [3], Proposition 7 for more details.

Proposition 9. (1) Let $f: \mathbb{R} \rightarrow \mathbb{R}$ be a continuous function with a constant $C>0$ such that $|f(m)| \leq C \exp (-\beta|m|)$ for all $m \in \mathbb{R}$, for some $\beta>0$. The inverse Fourier transform of $f$ is defined by the integral representation

$$
\mathscr{F}^{-1}(f)(x)=\frac{1}{(2 \pi)^{1 / 2}} \int_{-\infty}^{+\infty} f(m) \exp (i x m) d m
$$

for all $x \in \mathbb{R}$. It turns out that the function $\mathscr{F}^{-1}(f)$ extends to an analytic function on the horizontal strip

$$
H_{\beta}=\{z \in \mathbb{C}|| \operatorname{Im}(z) \mid<\beta\} .
$$

Let $\phi(m)=\operatorname{imf}(m)$. Then, we have the commuting relation

$$
\partial_{z} \mathscr{F}^{-1}(f)(z)=\mathscr{F}^{-1}(\phi)(z)
$$

for all $z \in H_{\beta}$.

(2) Let $f, g \in E_{(\beta, \mu)}$ and let $\psi(m)=\left(1 /(2 \pi)^{1 / 2}\right) f \star g(m)$, the convolution product of $f$ and $g$, for all $m \in \mathbb{R}$. From Proposition 2, we know that $\psi \in E_{(\beta, \mu)}$. Moreover, the following formula

$$
\mathscr{F}^{-1}(f)(z) \mathscr{F}^{-1}(g)(z)=\mathscr{F}^{-1}(\psi)(z)
$$

holds for all $z \in H_{\beta}$.

\section{Layout of the Main Nonlinear PDE and Related Auxiliary Problems}

Let $q, M, p \geq 0, D \geq 2$ be integers. For all $0 \leq l \leq q$, let $k_{l}, m_{l}$ be nonnegative integers and $a_{l}$ be complex numbers with $a_{0} \neq$ 0 such that $k_{l}<k_{l+1}$ for $l \in\{0, \ldots, q-1\}$. For all $0 \leq l \leq M$, we consider nonnegative integers $h_{l}, \mu_{l}$ and complex numbers $c_{l}$ with $c_{0} \neq 0$ such that $h_{l}<h_{l+1}$ for $l \in\{0, \ldots, M-1\}$. For all $0 \leq l \leq p$, we denote by $n_{l}$ and $b_{l}$ nonnegative integers such that $b_{l}<b_{l+1}$ for $l \in\{0, \ldots, p-1\}$. For $1 \leq l \leq D$, we set nonnegative integers $\Delta_{l}, d_{l}$, and $\delta_{l}$ such that $1 \leq \delta_{l}<\delta_{l+1}$ for $l \in\{1, \ldots, D-1\}$.

Let $Q(X), R_{l}(X) \in \mathbb{C}[X], 1 \leq l \leq D$, be polynomials which can be factorized as $Q(X)=X^{v} \widetilde{Q}(X), R_{l}(X)=$ $X^{v} \widetilde{R}_{l}(X)$, for some common integer $v \geq 1$, where $\widetilde{Q}(X)$ and $\widetilde{R}_{l}(X)$ are polynomials that satisfy

$$
\begin{aligned}
\operatorname{deg}(\widetilde{Q})=\operatorname{deg}\left(\widetilde{R}_{D}\right) \geq \operatorname{deg}\left(\widetilde{R}_{l}\right), \\
\qquad \widetilde{Q}(i m) \neq 0, \widetilde{R}_{D}(i m) \neq 0
\end{aligned}
$$

for all $m \in \mathbb{R}$ and all $1 \leq l \leq D-1$. 
We consider the following nonlinear singularly perturbed PDE:

$$
\begin{aligned}
& Q\left(\partial_{z}\right)\left(\left(\sum_{l=0}^{q} a_{l} \epsilon^{m_{l}} t^{k_{l}}\right) u(t, z, \epsilon)\right. \\
& \left.+\left(\sum_{l=0}^{M} c_{l} \epsilon^{\mu_{l}} t^{h_{l}}\right) u^{2}(t, z, \epsilon)\right)=\sum_{j=0}^{p} b_{j}(z) \epsilon^{n_{j}} t^{b_{j}} \\
& +\sum_{l=1}^{D} \epsilon^{\Delta_{l}} t^{d_{l}} \partial_{t}^{\delta_{l}} R_{l}\left(\partial_{z}\right) u(t, z, \epsilon) .
\end{aligned}
$$

The coefficients $b_{j}(z)$ are constructed as follows. For all $0 \leq$ $j \leq p$, we consider functions $m \mapsto \widetilde{B}_{j}(m)$ that belong to the Banach space $E_{(\beta, \mu)}$ for some $\mu>1$ and $\beta>0$. We define $B_{j}(m)=(i m)^{v} \widetilde{B}_{j}(m)$ where $v$ is the integer introduced above, for $0 \leq j \leq p$. We set

$$
b_{j}(z)=\mathscr{F}^{-1}\left(m \longmapsto B_{j}(m)\right)(z), \quad 0 \leq j \leq p,
$$

where $\mathscr{F}^{-1}$ denotes the Fourier inverse transform defined in Proposition 9. From (58), it turns out by construction that one can write $b_{j}(z)=\partial_{z}^{v} \widetilde{b}_{j}(z)$, where $\widetilde{b}_{j}(z)$ is the inverse Fourier transform of $\widetilde{B}_{j}(m)$.

Remark 10. The reason why we make these factorizations hypotheses on the polynomials $Q(X), R_{l}(X)$, and the functions $B_{j}(m)$ will be explained later on in Remark 14 of next section and is related to the construction of the Banach spaces in Section 2 and their Fourier inverse transforms.

Within this work, we will search for time rescaled solutions of (61) of the form

$$
u(t, z, \epsilon)=\epsilon^{\beta} U\left(\epsilon^{\alpha} t, z, \epsilon\right),
$$

where $\alpha, \beta \in \mathbb{Q}$ are two rational numbers and $\alpha>0$. Then, the expression $U(T, z, \epsilon)$ needs to formally solve the following nonlinear PDE:

$$
\begin{aligned}
Q\left(\partial_{z}\right)\left(\left(\sum_{l=0}^{q} a_{l} \epsilon^{m_{l}+\beta-\alpha k_{l}} T^{k_{l}}\right) U(T, z, \epsilon)\right. \\
\left.+\left(\sum_{l=0}^{M} c_{l} \epsilon^{\mu_{l}+2 \beta-\alpha h_{l}} T^{h_{l}}\right) U^{2}(T, z, \epsilon)\right) \\
=\sum_{j=0}^{p} b_{j}(z) \epsilon^{n_{j}-\alpha b_{j}} T^{b_{j}} \\
+\sum_{l=1}^{D} \epsilon^{\Delta_{l}+\alpha\left(\delta_{l}-d_{l}\right)+\beta} T^{d_{l}} R_{l}\left(\partial_{z}\right) \partial_{T}^{\delta_{l}} U(T, z, \epsilon) .
\end{aligned}
$$

4.1. Construction of a Distinguished Solution. We make the additional assumption that $\alpha, \beta$ set above can be chosen in such a way that the following inequalities

$$
\begin{array}{r}
\Delta_{l}+\alpha\left(\delta_{l}-d_{l}\right)+\beta>0, \\
n_{j}-\alpha b_{j}>0
\end{array}
$$

for all $1 \leq l \leq D, 0 \leq j \leq p$ and

$$
\begin{aligned}
& m_{l}+\beta-\alpha k_{l}=0, \\
& m_{j}+\beta-\alpha k_{j}>0
\end{aligned}
$$

for all $0 \leq l \leq s$ and all $s+1 \leq j \leq q$, for some integer $0 \leq s \leq q-1$, together with

$$
\begin{aligned}
& \mu_{l}+2 \beta-\alpha h_{l}=0 \\
& \mu_{j}+2 \beta-\alpha h_{j}>0
\end{aligned}
$$

for all $0 \leq l \leq s^{\prime}$ and all $s^{\prime}+1 \leq j \leq M$, for some integer $0 \leq s^{\prime} \leq M-1$, hold.

Remark 11. In the case $q=1, k_{0}, k_{1} \geq 1$, the roots of the polynomial (in $t$ ) $P(t, \epsilon)=a_{0} \epsilon^{m_{0}} t^{k_{0}}+a_{1} \epsilon^{m_{1}} t^{k_{1}}$ all have modulus equal to

$$
\left|\frac{a_{1}}{a_{0}}\right|^{1 /\left(k_{0}-k_{1}\right)}|\epsilon|^{\left(m_{1}-m_{0}\right) /\left(k_{0}-k_{1}\right)}
$$

except the trivial root 0 . The constraints (66) imply in particular that $m_{1}-m_{0}>\alpha\left(k_{1}-k_{0}\right)$. As a result, all the nonvanishing roots of $P(t, \epsilon)$ tend to $\infty$ as $\epsilon$ tends to 0 and 0 is therefore the only root (with order $k_{0}$ ) of $P(t, \epsilon)$ in the vicinity of 0 as $\epsilon$ stays near the origin.

Let us assume that the expression $U(T, z, \epsilon)$ is allowed to be written as a perturbation series with respect to $\epsilon$ :

$$
U(T, z, \epsilon)=U_{0}(T)+\sum_{n \geq 1} U_{n}(T, z) \epsilon^{n},
$$

where the constant term $U_{0}(T)$ is taken independently of $z$ and is not identically equal to 0 . The coefficient $U_{0}(T)$ is called the slow curve of (64) in the terminology of [6].

In the following, we make the assumption that $U_{0}(T)$ solves the following second-order algebraic equation:

$$
\left(\sum_{l=0}^{s} a_{l} T^{k_{l}}\right) U_{0}(T)+\left(\sum_{l=0}^{s^{\prime}} c_{l} T^{h_{l}}\right)\left(U_{0}(T)\right)^{2}=0 .
$$

As $U_{0}(T)$ is not identically vanishing, it must be equal to $-\left(\sum_{l=0}^{s} a_{l} T^{k_{l}}\right) /\left(\sum_{l=0}^{s^{\prime}} c_{l} T^{h_{l}}\right)$. Bearing in mind that $a_{0}, c_{0} \neq 0$, we get its asymptotic behaviour

$$
U_{0}(T) \sim-\frac{a_{0}}{c_{0}} T^{k_{0}-h_{0}}
$$

as $T$ tends to 0 .

Remark 12. Under the hypotheses (60) and (62), we observe, by factoring out the operator $\partial_{z}^{v}$ from (64), that $U(T, z, \epsilon)$ must solve the related PDE

$$
\begin{gathered}
\widetilde{Q}\left(\partial_{z}\right)\left(\left(\sum_{l=0}^{q} a_{l} \epsilon^{m_{l}+\beta-\alpha k_{l}} T^{k_{l}}\right) U(T, z, \epsilon)\right. \\
\left.+\left(\sum_{l=0}^{M} c_{l} \epsilon^{\mu_{l}+2 \beta-\alpha h_{l}} T^{h_{l}}\right) U^{2}(T, z, \epsilon)\right)
\end{gathered}
$$




$$
\begin{aligned}
& =\sum_{j=0}^{p} \widetilde{b}_{j}(z) \epsilon^{n_{j}-\alpha b_{j}} T^{b_{j}}+F(T, z, \epsilon) \\
& +\sum_{l=1}^{D} \epsilon^{\Delta_{l}+\alpha\left(\delta_{l}-d_{l}\right)+\beta} T^{d_{l}} \widetilde{R}_{l}\left(\partial_{z}\right) \partial_{T}^{\delta_{l}} U(T, z, \epsilon),
\end{aligned}
$$

where the forcing term $F(T, z, \epsilon)$ is a polynomial in $z$ of degree less than $v-1$. According to the assumptions (65), (66), and (67) and using the fact that $\widetilde{Q}(0) \neq 0$, by taking $\epsilon=0$ into (72), we see that the constraint (70) is equivalent to the fact that $F(T, z, 0) \equiv 0$. The precise shape of the term $F(T, z, \epsilon)$ will be given later in Section 6; see (183).

In a first step, we express $U(T, z, \epsilon)$ as a small perturbation of $U_{0}(T)$ that can be expressed in the form

$$
U_{0}(T)=-\frac{a_{0}}{c_{0}} T^{k_{0}-h_{0}}-\frac{a_{0}}{c_{0}} T^{k_{0}-h_{0}} J(T),
$$

where $J(T)=\sum_{j \geq 1} J_{j} T^{j}$ is a convergent series near $T=0$; namely,

$$
\begin{aligned}
U(T, z, \epsilon)= & -\frac{a_{0}}{c_{0}} T^{k_{0}-h_{0}}-\frac{a_{0}}{c_{0}} T^{k_{0}-h_{0}} J(T) \\
& +T^{\gamma} V(T, z, \epsilon)
\end{aligned}
$$

for some integer $\gamma \in \mathbb{Z}$ and some expression $V(T, x, \epsilon)$. By plugging this last expansion inside (64) and using the Leibniz rule, we get

$$
\begin{aligned}
Q & \left(\partial_{z}\right)\left(\left(\sum_{l=0}^{s} a_{l} T^{k_{l}}+\sum_{l=s+1}^{q} a_{l} \epsilon^{m_{l}+\beta-\alpha k_{l}} T^{k_{l}}\right)\right. \\
& \cdot\left(-\frac{a_{0}}{c_{0}} T^{k_{0}-h_{0}}-\frac{a_{0}}{c_{0}} T^{k_{0}-h_{0}} J(T)+T^{\gamma} V(T, z, \epsilon)\right) \\
+ & \left(\sum_{l=0}^{s^{\prime}} c_{l} T^{h_{l}}+\sum_{l=s^{\prime}+1}^{M} c_{l} \epsilon^{\mu_{l}+2 \beta-\alpha h_{l}} T^{h_{l}}\right) \\
& \left.\cdot\left(-\frac{a_{0}}{c_{0}} T^{k_{0}-h_{0}}-\frac{a_{0}}{c_{0}} T^{k_{0}-h_{0}} J(T)+T^{\gamma} V(T, z, \epsilon)\right)^{2}\right) \\
& =\sum_{j=0}^{p} b_{j}(z) \epsilon^{n_{j}-\alpha b_{j}} T^{b_{j}}+\sum_{l=1}^{D} \epsilon^{\Delta_{l}+\alpha\left(\delta_{l}-d_{l}\right)+\beta} T^{d_{l}} R_{l}\left(\partial_{z}\right) \\
& \cdot\left(-\frac{a_{0}}{c_{0}} \prod_{d=0}^{\delta_{l}-1}\left(k_{0}-h_{0}-d\right) T^{k_{0}-h_{0}-\delta_{l}}-\frac{a_{0}}{c_{0}}\right. \\
& \left.\sum_{q_{1}+q_{2}=\delta_{l}} \frac{\delta_{l} !}{q_{1} ! q_{2} !} \prod_{d=0}^{q_{1}-1}(\gamma-d) T^{\gamma-q_{1}} \partial_{T}^{q_{2}} V(T, z, \epsilon)\right) \\
& \sum_{q_{1}+q_{2}=\delta_{l}} \frac{\delta_{l} !}{q_{1} ! q_{2} !} \partial_{T}^{q_{1}}\left(T^{k_{0}-h_{0}}\right) \partial_{T}^{q_{2}} J(T)
\end{aligned}
$$

where we put $\prod_{d=0}^{-1}(\gamma-d)=1$ by convention. At this level, we observe the important fact that the coefficient in front of $Q\left(\partial_{z}\right) V(T, z, \epsilon)$ contains the term $a_{0} T^{k_{0}+\gamma}-$ $2\left(a_{0} / c_{0}\right) T^{k_{0}-h_{0}+\gamma} c_{0} T^{h_{0}}=-a_{0} T^{k_{0}+\gamma}$ that we want to set apart. As a result, we get the following equation satisfied by $V(T, z, \epsilon)$ :

$$
\begin{aligned}
Q & \left(\partial_{z}\right) V(T, z, \epsilon)\left(-a_{0} T^{k_{0}+\gamma}\right. \\
& +\left(\sum_{l=1}^{s} a_{l} T^{k_{l}}+\sum_{l=s+1}^{q} a_{l} \epsilon^{m_{l}+\beta-\alpha k_{l}} T^{k_{l}}\right) T^{\gamma}-2\left(\frac{a_{0}}{c_{0}}\right) \\
& \cdot T^{k_{0}-h_{0}+\gamma}\left(\sum_{l=1}^{s^{\prime}} c_{l} T^{h_{l}}+\sum_{l=s^{\prime}+1}^{M} c_{l} \epsilon^{\mu_{l}+2 \beta-\alpha h_{l}} T^{h_{l}}\right) \\
& -2\left(\frac{a_{0}}{c_{0}}\right) T^{k_{0}-h_{0}+\gamma} J(T) \\
& \left.\times\left(\sum_{l=0}^{s^{\prime}} c_{l} T^{h_{l}}+\sum_{l=s^{\prime}+1}^{M} c_{l} \epsilon^{\mu_{l}+2 \beta-\alpha h_{l}} T^{h_{l}}\right)\right)+Q\left(\partial_{z}\right) \\
& \cdot V^{2}(T, z, \epsilon) T^{2 \gamma}\left(\sum_{l=0}^{s_{1}^{\prime}} c_{l} T^{h_{l}}+\sum_{l=s^{\prime}+1}^{M} c_{l} \epsilon^{\mu_{l}+2 \beta-\alpha h_{l}} T^{h_{l}}\right) \\
& +\sum_{j=0}^{p} b_{j}(z) \epsilon^{n_{j}-\alpha b_{j}} T^{b_{j}}+\sum_{l=1}^{D} \epsilon^{\Delta_{l}+\alpha\left(\delta_{l}-d_{l}\right)+\beta} \\
& \cdot\left(\sum_{l} \frac{\delta_{l} !}{q_{1} ! q_{2} !}\right.
\end{aligned}
$$

We now introduce some additional constraints on the integers $\gamma, k_{l}, h_{j}, b_{h}$, for $0 \leq l \leq q, 0 \leq j \leq M, 0 \leq h \leq p$ and $d_{l}$, $\delta_{l}$, for $1 \leq l \leq D$. Namely, we impose that the following inequalities hold:

$$
k_{0}-h_{p} \leq \gamma
$$

for $0 \leq p \leq M$, together with

$$
\gamma \leq b_{j}-k_{0}
$$

for all $0 \leq j \leq p$ and finally

$$
\begin{aligned}
& k_{0}<d_{D}-\delta_{D}, \\
& k_{0} \leq d_{l}-\delta_{l}
\end{aligned}
$$

for all $1 \leq l \leq D-1$.

Remark 13. (1) For the case $k_{0}>h_{0}$, from (77), we need $\gamma \geq$ $k_{0}-h_{0}>0$. As a consequence of (78), we get that $b_{j}>k_{0}$, for $0 \leq j \leq p$. Let, for instance, $q=1, M=1, p=0$, and $D=2$. We set $\alpha=2, \beta=1, \gamma=6, \kappa=1$ and we choose the powers of $t$ and $\epsilon$ in the coefficients of (61) as follows:

$$
\begin{gathered}
m_{0}=3, \\
k_{0}=2,
\end{gathered}
$$




$$
\begin{aligned}
& m_{1}=6, \\
& k_{1}=3, \\
& \mu_{0}=0, \\
& h_{0}=1, \\
& \mu_{1}=3, \\
& h_{1}=2, \\
& n_{0}=19, \\
& b_{0}=9, \\
& \Delta_{1}=12, \\
& d_{1}=5, \\
& \delta_{1}=1, \\
& \Delta_{2}=20, \\
& d_{2}=6, \\
& \delta_{2}=2 .
\end{aligned}
$$

For these data, we can check that the constraints (65), (66), (67), (77), (78), and (79) above are fulfilled. Moreover, all the forthcoming requirements (88), (105), (106), (107), and (184) stated in Theorem 19 are also verified. In this special case, the main equation (61) writes

$$
\begin{aligned}
& Q\left(\partial_{z}\right)\left(\left(a_{0} \epsilon^{3} t^{2}+a_{1} \epsilon^{6} t^{3}\right) u(t, z, \epsilon)\right. \\
& \left.\quad+\left(c_{0} t+c_{1} \epsilon^{3} t^{2}\right) u^{2}(t, z, \epsilon)\right)=b_{0}(z) \epsilon^{19} t^{9} \\
& \quad+\epsilon^{12} t^{5} \partial_{t} R_{1}\left(\partial_{z}\right) u(t, z, \epsilon)+\epsilon^{20} t^{6} \partial_{t}^{2} R_{2}\left(\partial_{z}\right) u(t, z, \epsilon) .
\end{aligned}
$$

We can divide this last equation by $t$, but not by $\epsilon$, and the resulting equation still possesses a turning point and an irregular singularity at $t=0$.

(2) For the case $h_{0}>k_{0}$, we may take $\gamma<0$ and hence one can choose some $b_{j}<k_{0}$ for some $0 \leq j \leq p$. Let, for instance, $q=1, M=1, p=0$, and $D=2$. We choose $\alpha=2$, $\beta=-1, \gamma=-2$, and $\kappa=1$ and we select the powers of $t$ and $\epsilon$ in the coefficients of (61) as follows:

$$
\begin{aligned}
& m_{0}=5, \\
& k_{0}=2, \\
& m_{1}=10, \\
& k_{1}=4, \\
& \mu_{0}=14, \\
& h_{0}=6, \\
& \mu_{1}=19, \\
& h_{1}=8, \\
& n_{0}=3,
\end{aligned}
$$

$$
\begin{aligned}
& b_{0}=1, \\
& \Delta_{1}=10 \\
& d_{1}=5, \\
& \delta_{1}=1, \\
& \Delta_{2}=12, \\
& d_{2}=6 \\
& \delta_{2}=2 .
\end{aligned}
$$

For these data, we can figure out that the constraints (65), (66), (67), (77), (78), and (79) above are satisfied. Moreover, all the forthcoming requirements (88), (105), (106), (107), and (184) stated in Theorem 19 are also verified. In this particular case, the main equation (61) writes

$$
\begin{aligned}
& Q\left(\partial_{z}\right)\left(\left(a_{0} \epsilon^{5} t^{2}+a_{1} \epsilon^{10} t^{4}\right) u(t, z, \epsilon)\right. \\
& \left.\quad+\left(c_{0} \epsilon^{14} t^{6}+c_{1} \epsilon^{19} t^{8}\right) u^{2}(t, z, \epsilon)\right)=b_{0}(z) \epsilon^{3} t \\
& \quad+\epsilon^{10} t^{5} \partial_{t} R_{1}\left(\partial_{z}\right) u(t, z, \epsilon)+\epsilon^{12} t^{6} \partial_{t}^{2} R_{2}\left(\partial_{z}\right) u(t, z, \epsilon) .
\end{aligned}
$$

We can divide this latter equation by $\epsilon^{3}$ and by $t$. The corresponding equation still suffers the presence of a turning point and an irregular singularity at $t=0$.

In a second step, we divide the left- and right-hand sides of (76) by the monomial $T^{k_{0}+\gamma}$. We obtain the following equation:

$$
\begin{aligned}
Q\left(\partial_{z}\right) V(T, z, \epsilon)\left(-a_{0}\right. \\
+\left(\sum_{l=1}^{s} a_{l} T^{k_{l}}+\sum_{l=s+1}^{q} a_{l} \epsilon^{m_{l}+\beta-\alpha k_{l}} T^{k_{l}}\right) T^{-k_{0}}-2\left(\frac{a_{0}}{c_{0}}\right) \\
\cdot T^{-h_{0}}\left(\sum_{l=1}^{s^{\prime}} c_{l} T^{h_{l}}+\sum_{l=s^{\prime}+1}^{M} c_{l} \epsilon^{\mu_{l}+2 \beta-\alpha h_{l}} T^{h_{l}}\right)-2\left(\frac{a_{0}}{c_{0}}\right) \\
\left.+T^{-h_{0}} J(T) \times\left(\sum_{l=0}^{s^{\prime}} c_{l} T^{h_{l}}+\sum_{l=s^{\prime}+1}^{M} c_{l} \epsilon^{\mu_{l}+2 \beta-\alpha h_{l}} T^{h_{l}}\right)\right) \\
+Q\left(\partial_{z}\right) V^{2}(T, z, \epsilon) T^{-k_{0}+\gamma}\left(\sum_{l=0}^{s^{\prime}} c_{l} T^{h_{l}}\right. \\
\left.+\sum_{l=s^{\prime}+1}^{M} c_{l} \epsilon^{\mu_{l}+2 \beta-\alpha h_{l}} T^{h_{l}}\right)=\sum_{j=0}^{p} b_{j}(z) \epsilon^{n_{j}-\alpha b_{j}} T^{b_{j}-k_{0}-\gamma} \\
\left.+\sum_{l=1}^{D} \epsilon^{d_{l}-k_{0}-q_{1}} R_{l}\left(\partial_{z}\right) \partial_{T}^{q_{2}} V(T, z, \epsilon)\right) \\
\sum_{q_{1}+q_{2}=\delta_{l}} \frac{\delta_{l} !}{q_{1} ! q_{2} !} \prod_{d=0}^{q_{1}-1}(\gamma-d)
\end{aligned}
$$


Notice that the additional constraints (65), (66), (67) and (77), (78), (79) ensure that the coefficients of the PDE (84) are analytic with respect to $T$ and $\epsilon$ on a neighborhood of the origin in $\mathbb{C}^{2}$. Moreover, the coefficient of $Q\left(\partial_{z}\right) V(T, z, \epsilon)$ is invertible at $T=0$ since $a_{0} \neq 0$. We will see later that this fact is essential in order to solve this equation within some function space of analytic functions.

We look for solutions which are rescaled in time of the form

$$
V(T, z, \epsilon)=\mathbb{V}\left(\epsilon^{\chi} T, z, \epsilon\right),
$$

where

$$
\chi=\frac{\Delta_{D}+\alpha\left(\delta_{D}-d_{D}\right)+\beta}{d_{D}-k_{0}-\delta_{D}} .
$$

As a result, provided that $\mathbb{T}=\epsilon^{\chi} T$ holds, the expression $\mathbb{V}(\mathbb{T}, z, \epsilon)$ is supposed to solve the following equation:

$$
\begin{aligned}
& Q\left(\partial_{z}\right) \mathbb{V}(\mathbb{T}, z, \epsilon)\left(-a_{0}+\sum_{l=1}^{s} a_{l} \epsilon^{-\chi\left(k_{l}-k_{0}\right)} \mathbb{T}^{k_{l}-k_{0}}\right. \\
& +\sum_{l=s+1}^{q} a_{l} \epsilon^{m_{l}+\beta-\alpha k_{l}-\chi\left(k_{l}-k_{0}\right)} \mathbb{T}^{k_{l}-k_{0}}-2\left(\frac{a_{0}}{c_{0}}\right) \\
& \cdot\left(\sum_{l=1}^{s^{\prime}} c_{l} \epsilon^{-\chi\left(h_{l}-h_{0}\right)} \mathbb{T}^{h_{l}-h_{0}}\right. \\
& \left.+\sum_{l=s^{\prime}+1}^{M} c_{l} \epsilon^{\mu_{l}+2 \beta-\alpha h_{l}-\chi\left(h_{l}-h_{0}\right)} \mathbb{T}^{h_{l}-h_{0}}\right)-2\left(\frac{a_{0}}{c_{0}}\right) \\
& J\left(\epsilon^{-\chi} \mathbb{T}\right) \times\left(\sum_{l=0}^{s^{\prime}} c_{l} \epsilon^{-\chi\left(h_{l}-h_{0}\right)} \mathbb{T}^{h_{l}-h_{0}}\right. \\
& \left.\left.+\sum_{l=s^{\prime}+1}^{M} c_{l} \epsilon^{\mu_{l}+2 \beta-\alpha h_{l}-\chi\left(h_{l}-h_{0}\right)} \mathbb{T}^{h_{l}-h_{0}}\right)\right)+Q\left(\partial_{z}\right) \mathbb{V}^{2}(\mathbb{T}, \\
& z, \epsilon) \epsilon^{-\chi\left(-k_{0}+\gamma\right)} \mathbb{T}^{-k_{0}+\gamma}\left(\sum_{l=0}^{s^{\prime}} c_{l} \epsilon^{-\chi h_{l}} \mathbb{T}^{h_{l}}\right. \\
& \left.+\sum_{l=s^{\prime}+1}^{M} c_{l} \epsilon^{\mu_{l}+2 \beta-\alpha h_{l}-\chi h_{l}} \mathbb{T}^{h_{l}}\right)=\sum_{j=0}^{p} b_{j}(z) \\
& \cdot \epsilon^{n_{j}-\alpha b_{j}-\chi\left(b_{j}-k_{0}-\gamma\right)} \mathbb{T}^{b_{j}-k_{0}-\gamma}+\sum_{l=1}^{D-1} \epsilon^{\Delta_{l}+\alpha\left(\delta_{l}-d_{l}\right)+\beta} \\
& \times \sum_{q_{1}+q_{2}=\delta_{l}} \frac{\delta_{l} !}{q_{1} ! q_{2} !} \prod_{d=0}^{q_{1}-1}(\gamma-d) \epsilon^{-\chi\left(d_{l}-k_{0}-q_{1}\right)} \\
& \cdot \mathbb{T}^{d_{l}-k_{0}-q_{1}} R_{l}\left(\partial_{z}\right) \epsilon^{\chi q_{2}} \partial_{\mathbb{T}}^{q_{2}} \mathbb{V}(\mathbb{T}, z, \epsilon) \\
& +\epsilon^{\Delta_{D}+\alpha\left(\delta_{D}-d_{D}\right)+\beta} \sum_{q_{1}+q_{2}=\delta_{D}, q_{1} \geq 1} \frac{\delta_{D} !}{q_{1} ! q_{2} !} \prod_{d=0}^{q_{1}-1}(\gamma-d)
\end{aligned}
$$

$$
\begin{aligned}
& \cdot \epsilon^{-\chi\left(d_{D}-k_{0}-q_{1}\right)} \mathbb{T}^{d_{D}-k_{0}-q_{1}} \times R_{D}\left(\partial_{z}\right) \epsilon^{\chi q_{2}} \partial_{\mathbb{T}}^{q_{2}} \mathbb{V}(\mathbb{T}, z, \epsilon) \\
& +\mathbb{T}^{d_{D}-k_{0}} R_{D}\left(\partial_{z}\right) \partial_{\mathbb{T}}^{\delta_{D}} \mathbb{V}(\mathbb{T}, z, \epsilon) .
\end{aligned}
$$

We make further assumptions on the coefficients $d_{l}$ and $\delta_{l}$ for $1 \leq l \leq D$ which are stronger than the constraint (79). Assume the existence of integers $\kappa \geq 1$ and $d_{l, 0} \geq 1$ such that

$$
\begin{aligned}
& d_{D}-k_{0}=\delta_{D}(\kappa+1), \\
& d_{l}-k_{0}=\delta_{l}(\kappa+1)+d_{l, 0}
\end{aligned}
$$

for all $1 \leq l \leq D-1$. Then, for all $1 \leq l \leq D$, and all integers $q_{1} \geq 0, q_{2} \geq 0$ with $q_{1}+q_{2}=\delta_{l}$ we deduce the existence of a nonnegative integer $d_{l, q_{1}, q_{2}}$ which is larger than 1 except $d_{D, 0, \delta_{D}}=0$ such that

$$
d_{l}-k_{0}-q_{1}=(\kappa+1) q_{2}+d_{l, q_{1}, q_{2}} .
$$

Indeed, if one puts $d_{D, 0}=0$, from (88), we can write

$$
\begin{aligned}
d_{l, q_{1}, q_{2}} & =d_{l}-k_{0}-q_{1}-(\kappa+1) q_{2} \\
& =\delta_{l}(\kappa+1)+d_{l, 0}-q_{1}-(\kappa+1) q_{2} \\
& =\left(q_{1}+q_{2}\right)(\kappa+1)+d_{l, 0}-q_{1}-(\kappa+1) q_{2} \\
& =q_{1} \kappa+d_{l, 0} .
\end{aligned}
$$

According to (88) and (89), with the help of formula (8.7) from [26], p. 3630, we can expand the following pieces appearing in (87) satisfied by $\mathbb{V}(\mathbb{T}, z, \epsilon)$ :

$$
\begin{aligned}
& \mathbb{T}^{d_{D}-k_{0}} \partial_{\mathbb{T}}^{\delta_{D}} \mathbb{V}(\mathbb{T}, z, \epsilon)=\left(\left(\mathbb{T}^{\kappa+1} \partial_{\mathbb{T}}\right)^{\delta_{D}}\right. \\
& \left.+\sum_{1 \leq p \leq \delta_{D}-1} A_{\delta_{D}, p} \mathbb{T}^{\kappa\left(\delta_{D}-p\right)}\left(\mathbb{T}^{\kappa+1} \partial_{\mathbb{T}}\right)^{p}\right) \mathbb{V}(\mathbb{T}, z, \epsilon), \\
& \mathbb{T}^{d_{l}-k_{0}-\left(\delta_{l}-1\right)} \partial_{\mathbb{T}} \mathbb{V}(\mathbb{T}, z, \epsilon)=\mathbb{T}^{d_{l, \delta_{l}-1,1}}\left(\mathbb{T}^{\kappa+1} \partial_{\mathbb{T}}\right) \mathbb{V}(\mathbb{T}, z, \epsilon), \\
& \mathbb{T}^{d_{l}-k_{0}-q_{1}} \partial_{\mathbb{T}}^{q_{2}} \mathbb{V}(\mathbb{T}, z, \epsilon)=\mathbb{T}^{d_{l}, q_{1}, q_{2}} \mathbb{T}^{(\kappa+1) q_{2}} \partial_{\mathbb{T}}^{q_{2}} \mathbb{V}(\mathbb{T}, z, \epsilon) \\
& \quad=\mathbb{T}^{d_{l}, q_{1}, q_{2}}\left(\left(\mathbb{T}^{\kappa+1} \partial_{\mathbb{T}}\right)^{q_{2}}\right. \\
& \left.+\sum_{1 \leq p \leq q_{2}-1} A_{q_{2}, p} \mathbb{T}^{\kappa\left(q_{2}-p\right)}\left(\mathbb{T}^{\kappa+1} \partial_{\mathbb{T}}\right)^{p}\right) \mathbb{V}(\mathbb{T}, z, \epsilon)
\end{aligned}
$$

for all $1 \leq l \leq D$ and all integers $q_{1} \geq 0$ and $q_{2} \geq 2$ such that $q_{1}+q_{2}=\delta_{l}$, for some real constants $A_{\delta_{D}, p}, 1 \leq p \leq \delta_{D}-1$, and $A_{q_{2}, p}, 1 \leq p \leq q_{2}-1$.

In a third step, let us assume that the expression $\mathbb{V}(\mathbb{T}, z, \epsilon)$ has a formal power series expansion

$$
\mathbb{V}(\mathbb{T}, z, \epsilon)=\sum_{n \geq 1} \mathbb{V}_{n}(z, \epsilon) \mathbb{T}^{n}
$$


where each coefficient $\mathbb{V}_{n}(z, \epsilon)$ is defined as an inverse Fourier transform

$$
\mathbb{V}_{n}(z, \epsilon)=\mathscr{F}^{-1}\left(m \longmapsto \omega_{n}(m, \epsilon)\right)(z)
$$

for some function $m \mapsto \omega_{n}(m, \epsilon)$ belonging to the Banach space $E_{(\beta, \mu)}$ and depending holomorphically on $\epsilon$ on some punctured disc $D\left(0, \epsilon_{0}\right) \backslash\{0\}$ centered at 0 with radius $\epsilon_{0}>0$. We consider the formal power series

$$
\omega_{\kappa}(\tau, m, \epsilon)=\sum_{n \geq 1} \frac{\omega_{n}(m, \epsilon)}{\Gamma(n / \kappa)} \tau^{n}
$$

obtained by formally applying $m_{\kappa}$-Borel transform with respect to $\mathbb{T}$ and Fourier transform with respect to $z$ to the power series (92). The constraints (88) are introduced in such a way that $\omega_{\kappa}(\tau, m, \epsilon)$ satisfies some integral equation by making use of the properties of the $m_{\kappa}$-Borel transform of formal series and Fourier inverse transforms described in Propositions 8 and 9 with the help of the prepared expansions (91). Namely, after division by the power $(\mathrm{im})^{v}$, which is by construction a common factor of the functions $Q(\mathrm{im}), R_{l}(\mathrm{im})$, and $B_{j}(m)$ for $1 \leq l \leq D, 0 \leq j \leq p$, we get the new problem

$$
L_{\tau, m, \epsilon}\left(\omega_{\kappa}(\tau, m, \epsilon)\right)=R_{\tau, m, \epsilon}\left(\omega_{\kappa}(\tau, m, \epsilon)\right)
$$

$$
\begin{aligned}
& L_{\tau, m, \epsilon}\left(\omega_{\kappa}(\tau, m, \epsilon)\right)=\widetilde{Q}(i m)\left(-a_{0} \omega_{\kappa}(\tau, m, \epsilon)+\sum_{l=1}^{s} a_{l} \epsilon^{-\chi\left(k_{l}-k_{0}\right)} \frac{\tau^{\kappa}}{\Gamma\left(\left(k_{l}-k_{0}\right) / \kappa\right)} \int_{0}^{\tau^{\kappa}}\left(\tau^{\kappa}-s\right)^{\left(k_{l}-k_{0}\right) / \kappa-1} \omega_{\kappa}\left(s^{1 / \kappa}, m, \epsilon\right) \frac{d s}{s}\right. \\
& +\sum_{l=s+1}^{q} a_{l} \epsilon^{m_{l}+\beta-\alpha k_{l}-\chi\left(k_{1}-k_{0}\right)} \frac{\tau^{\kappa}}{\Gamma\left(\left(k_{l}-k_{0}\right) / \kappa\right)} \int_{0}^{\tau^{\kappa}}\left(\tau^{\kappa}-s\right)^{\left(k_{l}-k_{0}\right) / \kappa-1} \omega_{\kappa}\left(s^{1 / \kappa}, m, \epsilon\right) \frac{d s}{s}-2\left(\frac{a_{0}}{c_{0}}\right)\left(\sum_{l=1}^{s^{\prime}} c_{l} \epsilon^{-\chi\left(h_{l}-h_{0}\right)}\right. \\
& \frac{\tau^{\kappa}}{\Gamma\left(\left(h_{l}-h_{0}\right) / \kappa\right)} \int_{0}^{\tau^{\kappa}}\left(\tau^{\kappa}-s\right)^{\left(h_{l}-h_{0}\right) / \kappa-1} \omega_{\kappa}\left(s^{1 / \kappa}, m, \epsilon\right) \frac{d s}{s}+\sum_{l=s^{\prime}+1}^{M} c_{l} \epsilon^{\mu_{l}+2 \beta-\alpha h_{l}} \epsilon^{-\chi\left(h_{l}-h_{0}\right)} \frac{\tau^{\kappa}}{\Gamma\left(\left(h_{l}-h_{0}\right) / \kappa\right)} \int_{0}^{\tau^{\kappa}}\left(\tau^{\kappa}\right. \\
& \left.-s)^{\left(h_{l}-h_{0}\right) / \kappa-1} \omega_{\kappa}\left(s^{1 / \kappa}, m, \epsilon\right) \frac{d s}{s}\right)-2\left(\frac{a_{0}}{c_{0}}\right)\left(\sum_{l=0}^{s^{\prime}} c_{l} \sum_{j \geq 1} J_{j} \epsilon^{-\chi\left(h_{l}-h_{0}+j\right)} \frac{\tau^{\kappa}}{\Gamma\left(\left(h_{l}-h_{0}+j\right) / \kappa\right)} \int_{0}^{\tau^{\kappa}}\left(\tau^{\kappa}-s\right)^{\left(h_{l}-h_{0}+j\right) / \kappa-1}\right. \\
& \cdot \omega_{\kappa}\left(s^{1 / \kappa}, m, \epsilon\right) \frac{d s}{s}+\sum_{l=s^{\prime}+1}^{M} c_{l} \sum_{j \geq 1} J_{j} \epsilon^{\mu_{l}+2 \beta-\alpha h_{l}} \epsilon^{-\chi\left(h_{l}-h_{0}+j\right)} \frac{\tau^{\kappa}}{\Gamma\left(\left(h_{l}-h_{0}+j\right) / \kappa\right)} \int_{0}^{\tau^{\kappa}}\left(\tau^{\kappa}-s\right)^{\left(h_{l}-h_{0}+j\right) / \kappa-1} \\
& \left.\cdot \omega_{\kappa}\left(s^{1 / \kappa}, m, \epsilon\right) \frac{d s}{s}\right)+\widetilde{Q}(i m)\left(\sum_{l=0}^{s^{\prime}} \mathcal{c}_{l} \epsilon^{-\chi\left(-k_{0}+\gamma+h_{l}\right)} \frac{\tau^{\kappa}}{\Gamma\left(\left(-k_{0}+\gamma+h_{l}\right) / \kappa\right)} \int_{0}^{\tau^{\kappa}}\left(\tau^{\kappa}-s\right)^{\left(-k_{0}+\gamma+h_{l}\right) / \kappa-1}\right. \\
& \times\left\{s \int_{0}^{s} \int_{-\infty}^{+\infty} \frac{1}{(2 \pi)^{1 / 2}} \omega_{\kappa}\left(\left(s-s^{\prime}\right)^{1 / \kappa}, m-m_{1}, \epsilon\right) \omega_{\kappa}\left(\left(s^{\prime}\right)^{1 / \kappa}, m_{1}, \epsilon\right) \frac{1}{\left(s-s^{\prime}\right) s^{\prime}} d s^{\prime} d m_{1}\right\} \frac{d s}{s} \\
& +\sum_{l=s^{\prime}+1}^{M} c_{l} \epsilon^{\mu_{l}+2 \beta-\alpha h_{l}-\chi\left(h_{l}-k_{0}+\gamma\right)} \frac{\tau^{\kappa}}{\Gamma\left(\left(-k_{0}+\gamma+h_{l}\right) / \kappa\right)} \int_{0}^{\tau^{\kappa}}\left(\tau^{\kappa}-s\right)^{\left(-k_{0}+\gamma+h_{l}\right) / \kappa-1} \\
& \left.\times\left\{s \int_{0}^{s} \int_{-\infty}^{+\infty} \frac{1}{(2 \pi)^{1 / 2}} \omega_{\kappa}\left(\left(s-s^{\prime}\right)^{1 / \kappa}, m-m_{1}, \epsilon\right) \omega_{\kappa}\left(\left(s^{\prime}\right)^{1 / \kappa}, m_{1}, \epsilon\right) \frac{1}{\left(s-s^{\prime}\right) s^{\prime}} d s^{\prime} d m_{1}\right\} \frac{d s}{s}\right), \\
& R_{\tau, m, \epsilon}\left(\omega_{\kappa}(\tau, m, \epsilon)\right)=\sum_{j=0}^{p} \widetilde{B}_{j}(m) \epsilon^{n_{j}-\alpha b_{j}-\chi\left(b_{j}-k_{0}-\gamma\right)} \frac{\tau^{b_{j}-k_{0}-\gamma}}{\Gamma\left(\left(b_{j}-k_{0}-\gamma\right) / \kappa\right)}+\sum_{l=1}^{D-1} \epsilon^{\Delta_{l}+\alpha\left(\delta_{l}-d_{l}\right)+\beta} \times \sum_{q_{1}+q_{2}=\delta_{l}} \frac{\delta_{l} !}{q_{1} ! q_{2} !} \prod_{d=0}^{q_{1}-1}(\gamma-d) \\
& \cdot \epsilon^{-\chi\left(d_{l}-k_{0}-q_{1}-q_{2}\right)} \widetilde{R}_{l}(i m) \times\left\{\frac{\tau^{\kappa}}{\Gamma\left(d_{l, q_{1}, q_{2}} / \kappa\right)} \int_{0}^{\tau^{\kappa}}\left(\tau^{\kappa}-s\right)^{d_{l, q_{1}, q_{2}} / \kappa-1} \kappa^{q_{2}} s^{q_{2}} \omega_{\kappa}\left(s^{1 / \kappa}, m, \epsilon\right) \frac{d s}{s}+\sum_{1 \leq p \leq q_{2}-1} A_{q_{2}, p}\right. \\
& \left.\cdot \frac{\tau^{\kappa}}{\Gamma\left(\left(d_{l, q_{1}, q_{2}}+\kappa\left(q_{2}-p\right)\right) / \kappa\right)} \int_{0}^{\tau^{\kappa}}\left(\tau^{\kappa}-s\right)^{\left(d_{l, q_{1}, q_{2}}+\kappa\left(q_{2}-p\right)\right) / \kappa-1} \kappa^{p} s^{p} \omega_{\kappa}\left(s^{1 / \kappa}, m, \epsilon\right) \frac{d s}{s}\right\}+\epsilon^{\Delta_{D}+\alpha\left(\delta_{D}-d_{D}\right)+\beta}
\end{aligned}
$$




$$
\begin{aligned}
& \times \sum_{q_{1}+q_{2}=\delta_{D}, q_{1} \geq 1} \frac{\delta_{D} !}{q_{1} ! q_{2} !} \prod_{d=0}^{q_{1}-1}(\gamma-d) \epsilon^{-\chi\left(d_{D}-k_{0}-q_{1}-q_{2}\right)} \widetilde{R}_{D}(i m) \times\left\{\frac{\tau^{\kappa}}{\Gamma\left(d_{D, q_{1}, q_{2}} / \kappa\right)} \int_{0}^{\tau^{\kappa}}\left(\tau^{\mathcal{K}}-s\right)^{d_{D, q_{1}, q_{2}} / \kappa-1}\right. \\
& \cdot \kappa^{q_{2}} s^{q_{2}} \omega_{\kappa}\left(s^{1 / \kappa}, m, \epsilon\right) \frac{d s}{s}+\sum_{1 \leq p \leq q_{2}-1} A_{q_{2}, p} \frac{\tau^{\kappa}}{\Gamma\left(\left(d_{D, q_{1}, q_{2}}+\kappa\left(q_{2}-p\right)\right) / \kappa\right)} \int_{0}^{\tau^{\kappa}}\left(\tau^{\kappa}-s\right)^{\left(d_{D, q_{1}, q_{2}}+\kappa\left(q_{2}-p\right)\right) / \kappa-1} \\
& \left.\cdot \kappa^{p} s^{p} \omega_{\kappa}\left(s^{1 / \kappa}, m, \epsilon\right) \frac{d s}{s}\right\}+\widetilde{R}_{D}(i m)\left\{\left(\kappa \tau^{\kappa}\right)^{\delta_{D}} \omega_{\kappa}(\tau, m, \epsilon)+\sum_{1 \leq p \leq \delta_{D}-1} A_{\delta_{D}, p} \frac{\tau^{\kappa}}{\Gamma\left(\kappa\left(\delta_{D}-p\right) / \kappa\right)} \times \int_{0}^{\tau^{\kappa}}\left(\tau^{\kappa}\right.\right. \\
& \left.-s)^{\kappa\left(\delta_{D}-p\right) / \kappa-1} \kappa^{p} s^{p} \omega_{\kappa}\left(s^{1 / \kappa}, m, \epsilon\right) \frac{d s}{s}\right\} .
\end{aligned}
$$

By convention, the two sums $\sum_{1 \leq p \leq q_{2}-1}[\cdots]$ appearing in (97) are vanishing provided that $q_{2} \in\{0,1\}$.

Remark 14 . The hypotheses (60) and (62) ensure that (87) does not contain terms that involve isolated polynomials in $\mathbb{}$ which are not inverse Fourier transformable.

\section{Analytic Solutions of a Convolution Problem with Complex Parameters}

Our main goal in this section is the construction of a unique solution of problem (95) within the Banach spaces introduced in Section 2.

We make the following further assumptions. The conditions below are very similar to the ones proposed in Section 4 of [3]. Namely, we demand that there exists an unbounded sector

$$
\begin{aligned}
& S_{\widetilde{\mathrm{Q}}, \widetilde{R}_{D}} \\
& \quad=\left\{z \in \mathbb{C}|| z\left|\geq r_{\widetilde{\mathrm{Q}}, \widetilde{R}_{D}},\right| \arg (z)-d_{\widetilde{\mathrm{Q}}, \widetilde{R}_{D}} \mid \leq \eta_{\widetilde{\mathrm{Q}}, \widetilde{R}_{D}}\right\}
\end{aligned}
$$

with direction $d_{\widetilde{\mathrm{Q}}, \widetilde{R}_{D}} \in \mathbb{R}$ and aperture $\eta_{\widetilde{\mathrm{Q}}_{,}, \widetilde{R}_{\mathrm{D}}}>0$ for some radius $r_{\widetilde{Q}, \widetilde{R}_{D}}>0$ such that

$$
\frac{\widetilde{\mathrm{Q}}(i m)}{\widetilde{R}_{D}(i m)} \in S_{\widetilde{\mathrm{Q}}, \widetilde{R}_{D}}
$$

for all $m \in \mathbb{R}$. The polynomial $\widetilde{P}_{m}(\tau)=-\widetilde{Q}(i m) a_{0}-$ $\widetilde{R}_{D}(i m) \kappa^{\delta_{D}} \tau^{\delta_{D} \kappa}$ can be factorized in the form

$$
\widetilde{P}_{m}(\tau)=-\widetilde{R}_{D}(i m) \kappa^{\delta_{D}} \prod_{l=0}^{\delta_{D} \kappa-1}\left(\tau-q_{l}(m)\right),
$$

where

$$
\begin{aligned}
& q_{l}(m)=\left(\frac{\left|a_{0} \widetilde{Q}(i m)\right|}{\left|\widetilde{R}_{D}(i m)\right| \kappa^{\delta_{D}}}\right)^{1 / \delta_{D} \kappa} \\
& \quad \cdot \exp \left(\sqrt{-1}\left(\arg \left(\frac{-a_{0} \widetilde{Q}(i m)}{\widetilde{R}_{D}(i m) \kappa^{\delta_{D}}}\right) \frac{1}{\delta_{D} \kappa}+\frac{2 \pi l}{\delta_{D} \kappa}\right)\right)
\end{aligned}
$$

for all $0 \leq l \leq \delta_{D} \kappa-1$ and all $m \in \mathbb{R}$.
We select an unbounded sector $S_{d}$ centered at 0 and a small closed disc $\bar{D}(0, \rho)$ and we require the sector $S_{\widetilde{Q}^{,}, \widetilde{R}_{D}}$ to fulfill the next conditions.

(1) There exists a constant $M_{1}>0$ such that

$$
\left|\tau-q_{l}(m)\right| \geq M_{1}(1+|\tau|)
$$

for all $0 \leq l \leq \delta_{D} \kappa-1$, all $m \in \mathbb{R}$, and all $\tau \in S_{d} \cup \bar{D}(0, \rho)$. Indeed, from (99) and the explicit expression (101) of $q_{l}(m)$, we first observe that $\left|q_{l}(m)\right|>2 \rho$ for every $m \in \mathbb{R}$, all $0 \leq l \leq$ $\delta_{D} \kappa-1$ for an appropriate choice of $r_{\widetilde{\mathrm{Q}}, \widetilde{R}_{D}}$ and of $\rho>0$. We also see that, for all $m \in \mathbb{R}$ and all $0 \leq l \leq \delta_{D} \kappa-1$, the roots $q_{l}(m)$ remain in a union $\mathcal{U}$ of unbounded sectors centered at 0 that do not cover a full neighborhood of the origin in $\mathbb{C}^{*}$ provided that $\eta_{\widetilde{Q}, \widetilde{R}_{D}}$ is small enough. Therefore, one can choose an adequate sector $S_{d}$ such that $S_{d} \cap \mathcal{U}=\emptyset$ with the property that for all $0 \leq l \leq \delta_{D} \kappa-1$ the quotients $q_{l}(m) / \tau$ lay outside some small disc centered at 1 in $\mathbb{C}$ for all $\tau \in S_{d}$ and all $m \in \mathbb{R}$. This yields (102) for some small constant $M_{1}>0$.

(2) There exists a constant $M_{2}>0$ such that

$$
\left|\tau-q_{l_{0}}(m)\right| \geq M_{2}\left|q_{l_{0}}(m)\right|
$$

for some $l_{0} \in\left\{0, \ldots, \delta_{D} \kappa-1\right\}$, all $m \in \mathbb{R}$, and all $\tau \in S_{d} \cup$ $\bar{D}(0, \rho)$. Indeed, for the sector $S_{d}$ and the disc $\bar{D}(0, \rho)$ chosen as above in (1), we notice that for any fixed $0 \leq l_{0} \leq \delta_{D} \kappa-1$, the quotient $\tau / q_{l_{0}}(m)$ stays outside a small disc centered at 1 in $\mathbb{C}$ for all $\tau \in S_{d} \cup \bar{D}(0, \rho)$ and all $m \in \mathbb{R}$. Hence (103) must hold for some small constant $M_{2}>0$.

By construction of the roots (101) in the factorization (100) and using the lower bound estimates (102) and (103), we get a constant $C_{\widetilde{P}}>0$ such that

$$
\begin{aligned}
& \left|\widetilde{P}_{m}(\tau)\right| \geq M_{1}^{\delta_{D} \kappa-1} M_{2}\left|\widetilde{R}_{D}(i m) \kappa^{\delta_{D}}\right| \\
& \cdot\left(\frac{\left|a_{0} \widetilde{Q}(i m)\right|}{\left|\widetilde{R}_{D}(i m)\right| \kappa^{\delta_{D}}}\right)^{1 / \delta_{D} \kappa}(1+|\tau|)^{\delta_{D} \kappa-1}
\end{aligned}
$$




$$
\begin{aligned}
& \geq M_{1}^{\delta_{D} \kappa-1} M_{2} \frac{\kappa^{\delta_{D}}\left|a_{0}\right|^{1 / \delta_{D} \kappa}}{\left(\kappa^{\delta_{D}}\right)^{1 / \delta_{D} \kappa}}\left(r_{\widetilde{Q}, \widetilde{R}_{D}}\right)^{1 / \delta_{D} \kappa}\left|\widetilde{R}_{D}(i m)\right| \\
& \times\left(\min _{x \geq 0} \frac{(1+x)^{\delta_{D^{\kappa}-1}}}{\left(1+x^{\kappa}\right)^{\delta_{D}-1 / \kappa}}\right)\left(1+|\tau|^{\kappa}\right)^{\delta_{D}-1 / \kappa} \\
& =C_{\widetilde{P}}\left(r_{\widetilde{\mathrm{Q}}, \widetilde{R}_{D}}\right)^{1 / \delta_{D} \kappa}\left|\widetilde{R}_{D}(i m)\right|\left(1+|\tau|^{\kappa}\right)^{\delta_{D}-1 / \kappa}
\end{aligned}
$$

for all $\tau \in S_{d} \cup \bar{D}(0, \rho)$ and all $m \in \mathbb{R}$.

In the next proposition, we provide sufficient conditions under which the main convolution equation (95) possesses solutions $\omega_{\kappa}^{d}(\tau, m, \epsilon)$ in the Banach space $F_{(\nu, \beta, \mu, \chi, \alpha, \kappa, \epsilon)}^{d}$ described in Section 2.

Proposition 15. Under the additional assumptions

$$
\begin{aligned}
& (\chi+\alpha)\left(-k_{0}+\gamma+h_{l}-\kappa \delta_{D}+1\right)-\chi\left(-k_{0}+\gamma+h_{l}\right) \\
& \quad \geq 0
\end{aligned}
$$

$$
\delta_{D} \geq \frac{2}{\kappa},-k_{0}+\gamma+h_{l}>0, b_{j}-k_{0}-\gamma \geq 1 \text {, }
$$

for all $0 \leq l \leq M, 0 \leq j \leq p$,

$$
\begin{aligned}
\Delta_{l}+ & \alpha\left(\delta_{l}-d_{l}\right)+\beta \\
& +(\chi+\alpha) \kappa\left(\frac{d_{l, q_{1}, q_{2}}}{\kappa}+q_{2}-\delta_{D}+\frac{1}{\kappa}\right) \\
& -\chi\left(d_{l}-k_{0}-\delta_{l}\right) \geq 0, \quad \delta_{D} \geq \frac{1}{\kappa}+\delta_{l}
\end{aligned}
$$

$$
\begin{aligned}
& \mathscr{H}_{\epsilon}^{1}(w(\tau, m)):=\sum_{j=0}^{p} \widetilde{B}_{j}(m) \epsilon^{n_{j}-\alpha b_{j}-\chi\left(b_{j}-k_{0}-\gamma\right)} \frac{\tau^{b_{j}-k_{0}-\gamma}}{\widetilde{P}_{m}(\tau) \Gamma\left(\left(b_{j}-k_{0}-\gamma\right) / \kappa\right)}-\widetilde{Q}(i m)\left(\sum_{l=1}^{s} a_{l} \epsilon^{-\chi\left(k_{l}-k_{0}\right)} \frac{\tau^{\kappa}}{\widetilde{P}_{m}(\tau) \Gamma\left(\left(k_{l}-k_{0}\right) / \kappa\right)}\right. \\
& \cdot \int_{0}^{\tau^{\kappa}}\left(\tau^{\kappa}-s\right)^{\left(k_{l}-k_{0}\right) / \kappa-1} w\left(s^{1 / \kappa}, m\right) \frac{d s}{s}+\sum_{l=s+1}^{q} a_{l} \epsilon^{m_{l}+\beta-\alpha k_{l}-\chi\left(k_{l}-k_{0}\right)} \frac{\tau^{\kappa}}{\widetilde{P}_{m}(\tau) \Gamma\left(\left(k_{l}-k_{0}\right) / \kappa\right)} \int_{0}^{\tau^{\kappa}}\left(\tau^{\kappa}-s\right)^{\left(k_{l}-k_{0}\right) / \kappa-1} \\
& \cdot w\left(s^{1 / \kappa}, m\right) \frac{d s}{s}-2\left(\frac{a_{0}}{c_{0}}\right)\left(\sum_{l=1}^{s_{l}^{\prime}} c_{l} \epsilon^{-\chi\left(h_{l}-h_{0}\right)} \frac{\tau^{\kappa}}{\widetilde{P}_{m}(\tau) \Gamma\left(\left(h_{l}-h_{0}\right) / \kappa\right)} \int_{0}^{\tau^{\kappa}}\left(\tau^{\kappa}-s\right)^{\left(h_{l}-h_{0}\right) / \kappa-1} w\left(s^{1 / \kappa}, m\right) \frac{d s}{s}\right. \\
& \left.+\sum_{l=s^{\prime}+1}^{M} c_{l} \epsilon^{\mu_{l}+2 \beta-\alpha h_{l}} \epsilon^{-\chi\left(h_{l}-h_{0}\right)} \frac{\tau^{\kappa}}{\widetilde{P}_{m}(\tau) \Gamma\left(\left(h_{l}-h_{0}\right) / \kappa\right)} \int_{0}^{\tau^{\kappa}}\left(\tau^{\kappa}-s\right)^{\left(h_{l}-h_{0}\right) / \kappa-1} w\left(s^{1 / \kappa}, m\right) \frac{d s}{s}\right)-2\left(\frac{a_{0}}{c_{0}}\right)\left(\sum_{l=0}^{s^{\prime}} c_{l}\right. \\
& \cdot \sum_{j \geq 1} J_{j} \epsilon^{-\chi\left(h_{l}-h_{0}+j\right)} \frac{\tau^{\kappa}}{\widetilde{P}_{m}(\tau) \Gamma\left(\left(h_{l}-h_{0}+j\right) / \kappa\right)} \int_{0}^{\tau^{\kappa}}\left(\tau^{\kappa}-s\right)^{\left(h_{l}-h_{0}+j\right) / \kappa-1} w\left(s^{1 / \kappa}, m\right) \frac{d s}{s}+\sum_{l=s^{\prime}+1}^{M} c_{j} \sum_{j \geq 1} J_{j} \epsilon^{\mu_{l}+2 \beta-\alpha h_{l}} \epsilon^{-\chi\left(h_{l}-h_{0}+j\right)} \\
& \left.\left.\quad \times \frac{\tau^{\kappa}}{\widetilde{P}_{m}(\tau) \Gamma\left(\left(h_{l}-h_{0}+j\right) / \kappa\right)} \int_{0}^{\tau^{\kappa}}\left(\tau^{\kappa}-s\right)^{\left(h_{l}-h_{0}+j\right) / \kappa-1} w\left(s^{1 / \kappa}, m\right) \frac{d s}{s}\right)\right),
\end{aligned}
$$




$$
\begin{aligned}
& \mathscr{H}_{\epsilon}^{2}(w(\tau, m)):=-\widetilde{Q}(i m)\left(\sum_{l=0}^{s^{\prime}} c_{l} \epsilon^{-\chi\left(-k_{0}+\gamma+h_{l}\right)} \frac{\tau^{\kappa}}{\widetilde{P}_{m}(\tau) \Gamma\left(\left(-k_{0}+\gamma+h_{l}\right) / \kappa\right)} \int_{0}^{\tau^{\kappa}}\left(\tau^{\kappa}-s\right)^{\left(-k_{0}+\gamma+h_{l}\right) / \kappa-1}\right. \\
& \quad \times\left\{s \int_{0}^{s} \int_{-\infty}^{+\infty} \frac{1}{(2 \pi)^{1 / 2}} w\left(\left(s-s^{\prime}\right)^{1 / \kappa}, m-m_{1}\right) w\left(\left(s^{\prime}\right)^{1 / \kappa}, m_{1}\right) \frac{1}{\left(s-s^{\prime}\right) s^{\prime}} d s^{\prime} d m_{1}\right\} \frac{d s}{s}+\sum_{l=s^{\prime}+1}^{M} c_{l} \epsilon^{\mu_{l}+2 \beta-\alpha h_{l}-\chi\left(h_{l}-k_{0}+\gamma\right)} \\
& \quad \frac{\tau^{\kappa}}{\widetilde{P}_{m}(\tau) \Gamma\left(\left(-k_{0}+\gamma+h_{l}\right) / \kappa\right)} \int_{0}^{\tau^{\kappa}}\left(\tau^{\kappa}-s\right)^{\left(-k_{0}+\gamma+h_{l}\right) / \kappa-1} \\
& \left.\quad \times\left\{s \int_{0}^{s} \int_{-\infty}^{+\infty} \frac{1}{(2 \pi)^{1 / 2}} w\left(\left(s-s^{\prime}\right)^{1 / \kappa}, m-m_{1}\right) w\left(\left(s^{\prime}\right)^{1 / \kappa}, m_{1}\right) \frac{1}{\left(s-s^{\prime}\right) s^{\prime}} d s^{\prime} d m_{1}\right\} \frac{d s}{s}\right)+\sum_{l=1}^{D-1} \epsilon^{\Delta_{l}+\alpha\left(\delta_{l}-d_{l}\right)+\beta} \\
& \quad \times \sum_{q_{1}+q_{2}=\delta_{l}} \frac{\delta_{l} !}{q_{1} ! q_{2} !} \prod_{d=0}^{q_{1}-1}(\gamma-d) \epsilon^{-\chi\left(d_{l}-k_{0}-q_{1}-q_{2}\right)} \widetilde{R}_{l}(i m) \times\left\{\frac{\tau^{\kappa}}{\widetilde{P}_{m}(\tau) \Gamma\left(d_{l, q_{1}, q_{2}} / \kappa\right)} \int_{0}^{\tau^{\kappa}}\left(\tau^{\kappa}-s\right)^{d_{l, q_{1}, q_{2}} / \kappa-1} \kappa^{q_{2}} s^{q_{2}} w\left(s^{1 / \kappa}, m\right) \frac{d s}{s}\right. \\
& \left.\quad+\sum_{1 \leq p \leq q_{2}-1} A_{q_{2}, p} \frac{\tau^{\kappa}}{\widetilde{P}_{m}(\tau) \Gamma\left(\left(d_{l, q_{1}, q_{2}}+\kappa\left(q_{2}-p\right)\right) / \kappa\right)} \int_{0}^{\tau^{\kappa}}\left(\tau^{\kappa}-s\right)^{\left(d_{l, q_{1}, q_{2}}+\kappa\left(q_{2}-p\right)\right) / \kappa-1} \kappa^{p} s^{p} w\left(s^{1 / \kappa}, m\right) \frac{d s}{s}\right\},
\end{aligned}
$$

along with

$$
\begin{aligned}
& \mathscr{H}_{\epsilon}^{3}(w(\tau, m)):=\epsilon^{\Delta_{D}+\alpha\left(\delta_{D}-d_{\mathrm{D}}\right)+\beta} \times \sum_{q_{1}+q_{2}=\delta_{D}, q_{1} \geq 1} \frac{\delta_{D} !}{q_{1} ! q_{2} !} \\
& \cdot \prod_{d=0}^{q_{1}-1}(\gamma-d) \epsilon^{-\chi\left(d_{D}-k_{0}-q_{1}-q_{2}\right)} \widetilde{R}_{D}(i m) \\
& \times\left\{\frac{\tau^{\kappa}}{\widetilde{P}_{m}(\tau) \Gamma\left(d_{D, q_{1}, q_{2}} / \kappa\right)} \int_{0}^{\tau^{\kappa}}\left(\tau^{\kappa}-s\right)^{d_{D, q_{1}, q_{2}} / \kappa-1}\right. \\
& \cdot \kappa^{q_{2}} s^{q_{2}} w\left(s^{1 / \kappa}, m\right) \frac{d s}{s}+\sum_{1 \leq p \leq q_{2}-1} A_{q_{2}, p} \\
& \cdot \frac{\tau^{\kappa}}{\widetilde{P}_{m}(\tau) \Gamma\left(\left(d_{D, q_{1}, q_{2}}+\kappa\left(q_{2}-p\right)\right) / \kappa\right)} \\
& \cdot \int_{0}^{\tau^{\kappa}}\left(\tau^{\kappa}-s\right)^{\left(d_{D, q_{1}, q_{2}}+\kappa\left(q_{2}-p\right)\right) / \kappa-1} \\
& \left.\cdot \kappa^{p} s^{p} w\left(s^{1 / \kappa}, m\right) \frac{d s}{s}\right\}+\widetilde{R}_{D}(i m) \\
& \left\{\sum_{1 \leq p \leq \delta_{D}-1} A_{\delta_{D}, p} \frac{\tau^{\kappa}}{\widetilde{P}_{m}(\tau) \Gamma\left(\kappa\left(\delta_{D}-p\right) / \kappa\right)}\right. \\
& \left.\times \int_{0}^{\tau^{\kappa}}\left(\tau^{\kappa}-s\right)^{\kappa\left(\delta_{D}-p\right) / \kappa-1} \kappa^{p} s^{p} w\left(s^{1 / \kappa}, m\right) \frac{d s}{s}\right\},
\end{aligned}
$$

satisfies the next properties.

(i) The following inclusion holds:

$$
\mathscr{H}_{\epsilon}(\bar{B}(0, \omega)) \subset \bar{B}(0, \varpi),
$$

where $\bar{B}(0, \emptyset)$ is the closed ball of radius $\omega>0$ centered at 0 in $F_{(\nu, \beta, \mu, \chi, \alpha, \kappa, \epsilon)}^{d}$, for all $\epsilon \in D\left(0, \epsilon_{0}\right) \backslash\{0\}$.

(ii) One has

$$
\begin{gathered}
\left\|\mathscr{H}_{\epsilon}\left(w_{1}\right)-\mathscr{H}_{\epsilon}\left(w_{2}\right)\right\|_{(\nu, \beta, \mu, \chi, \alpha, \alpha, \epsilon)} \\
\leq \frac{1}{2}\left\|w_{1}-w_{2}\right\|_{(\nu, \beta, \mu, \chi, \alpha, \kappa, \epsilon)}
\end{gathered}
$$

for all $w_{1}, w_{2} \in \bar{B}(0, \omega)$, for all $\epsilon \in D\left(0, \epsilon_{0}\right) \backslash\{0\}$.

Proof. We first deal with the property (113). Let $\epsilon \in D\left(0, \epsilon_{0}\right) \backslash$ $\{0\}$ and consider $w(\tau, m) \in F_{(\nu, \beta, \mu, \chi, \alpha, \kappa, \epsilon)}^{d}$. We take $\Phi>0$ such that $\|w(\tau, m)\|_{(\nu, \beta, \mu, \chi, \alpha, \kappa, \epsilon)} \leq \omega$.

We start providing norm estimates for each piece of the map $\mathscr{H}_{\epsilon}^{1}$.

From Lemma 4, we deduce the existence of a constant $C_{1}>0$ depending on $\kappa, \gamma, k_{0}$, and $b_{j}$ for $0 \leq j \leq p$ such that

$$
\begin{gathered}
\left\|\widetilde{B}_{j}(m) \epsilon^{-\chi\left(b_{j}-k_{0}-\gamma\right)} \frac{\tau^{b_{j}-k_{0}-\gamma}}{\widetilde{P}_{m}(\tau)}\right\|_{(\nu, \beta, \mu, \chi, \alpha, \kappa, \epsilon)} \\
\leq \frac{C_{1}}{C_{\widetilde{P}}\left(r_{\widetilde{\mathrm{Q}}, \widetilde{R}_{D}}\right)^{1 / \delta_{D} \kappa}} \frac{\left\|\widetilde{B}_{j}(m)\right\|_{(\beta, \mu)}}{\inf _{m \in \mathbb{R}}\left|\widetilde{R}_{D}(i m)\right|}|\epsilon|^{\left(b_{j}-k_{0}-\gamma\right) \alpha} .
\end{gathered}
$$

According to Proposition 5(1), we obtain a constant $C_{2}>0$ depending on $\nu, \kappa, k_{l}$, for $0 \leq l \leq q, h_{l}$, for $0 \leq l \leq M$, $\widetilde{Q}(X), \widetilde{R}_{D}(X)$, and a constant $C_{2}(j)$ depending on $\nu, \kappa, h_{l}$ for $0 \leq l \leq M, \widetilde{Q}(X), \widetilde{R}_{D}(X)$ and $j$ such that

$$
\| \epsilon^{-\chi\left(k_{l}-k_{0}\right)} \frac{\widetilde{Q}(i m) \tau^{\kappa}}{\widetilde{P}_{m}(\tau)} \int_{0}^{\tau^{\kappa}}\left(\tau^{\kappa}-s\right)^{\left(k_{l}-k_{0}\right) / \kappa-1}
$$




$$
\begin{aligned}
& \cdot w\left(s^{1 / \kappa}, m\right) \frac{d s}{s} \|_{(\nu, \beta, \mu, \chi, \alpha, \kappa, \epsilon)} \\
& \leq \frac{C_{2}}{C_{\widetilde{P}}\left(r_{\widetilde{Q}, \widetilde{R}_{D}}\right)^{1 / \delta_{D} \kappa}}|\epsilon|^{\alpha\left(k_{l}-k_{0}\right)}\|w(\tau, m)\|_{(\nu, \beta, \mu, \chi, \alpha, \kappa, \epsilon)}, \\
& \| \epsilon^{-\chi\left(h_{l}-h_{0}\right)} \frac{\widetilde{Q}(i m) \tau^{\kappa}}{\widetilde{P}_{m}(\tau)} \int_{0}^{\tau^{\kappa}}\left(\tau^{\kappa}-s\right)^{\left(h_{l}-h_{0}\right) / \kappa-1} \\
& \cdot w\left(s^{1 / \kappa}, m\right) \frac{d s}{s} \|_{(\nu, \beta, \mu, \chi, \alpha, \kappa, \epsilon)} \\
& \leq \frac{C_{2}}{C_{\widetilde{P}}\left(r_{\widetilde{Q}, \widetilde{R}_{D}}\right)^{1 / \delta_{D} \kappa}}|\epsilon|^{\alpha\left(h_{l}-h_{0}\right)}\|w(\tau, m)\|_{(\nu, \beta, \mu, \chi, \alpha, \kappa, \epsilon)}, \\
& \| \epsilon^{-\chi\left(h_{l}-h_{0}+j\right)} \frac{\widetilde{Q}(i m)}{\widetilde{P}_{m}(\tau)} \tau^{\kappa} \int_{0}^{\tau^{\kappa}}\left(\tau^{\kappa}-s\right)^{\left(h_{l}-h_{0}+j\right) / \kappa-1} \\
& \cdot w\left(s^{1 / \kappa}, m\right) \frac{d s}{s} \|_{(\nu, \beta, \mu, \chi, \alpha, \kappa, \epsilon)} \\
& \leq \frac{C_{2}(j)}{C_{\widetilde{P}}\left(r_{\widetilde{Q}, \widetilde{R}_{\mathrm{D}}}\right)^{1 / \delta_{D} \kappa}}|\epsilon|^{\alpha\left(h_{l}-h_{0}+j\right)}\|w(\tau, m)\|_{(\nu, \beta, \mu, \chi, \alpha, \kappa, \epsilon)} .
\end{aligned}
$$

Moreover, it appears from the proof of Proposition 5 that the next bounds hold for $C_{2}(j)$ : there exist a constant $\widehat{C}_{2}>0$ depending on $\nu, \kappa, h_{l}$ for $0 \leq l \leq M, \widetilde{Q}, \widetilde{R}_{D}$ and a constant $A_{2}>0$ depending on $\nu, \kappa, h_{l}$ for $0 \leq l \leq M$ such that

$$
C_{2}(j) \leq \widehat{C}_{2} A_{2}^{j} \Gamma\left(\frac{h_{l}-h_{0}+j}{\kappa}\right)
$$

for all $j \geq 1$. In the following, we will make use of the notations from the proof of Proposition 5. From the classical estimates

$$
\sup _{x \geq 0} x^{m_{1}} e^{-m_{2} x}=\left(\frac{m_{1}}{m_{2}}\right)^{m_{1}} e^{-m_{1}}
$$

for any real numbers $m_{1} \geq 0, m_{2}>0$, we deduce that for all $j \geq 1$ such that $\left(h_{l}-h_{0}+j\right) / \kappa-1>0$

$$
\begin{aligned}
& \sup _{x \geq 0} \frac{1+x^{2}}{x^{1 / \kappa}} e^{-v x} x G_{1}(x) \leq \sup _{x \geq 0}\left(1+x^{2}\right) \\
& \cdot x^{\left(h_{l}-h_{0}+j\right) / \kappa} e^{-(\nu / 2) x}\left(\frac{1}{2}\right)^{1 / \kappa} \kappa \\
& \leq\left(\left(\frac{h_{l}-h_{0}+j}{\kappa \nu / 2}\right)^{\left(h_{l}-h_{0}+j\right) / \kappa} \exp \left(-\frac{h_{l}-h_{0}+j}{\kappa}\right)\right. \\
&+\left(\frac{\left(h_{l}-h_{0}+j\right) / \kappa+2}{\nu / 2}\right)^{\left(h_{l}-h_{0}+j\right) / \kappa+2} \\
&\left.\cdot \exp \left(-\left(\frac{h_{l}-h_{0}+j}{\kappa}+2\right)\right)\right)\left(\frac{1}{2}\right)^{1 / \kappa} \kappa .
\end{aligned}
$$

Furthermore, according to the Stirling formula $\Gamma(x)$ $\sqrt{2 \pi} x^{x-1 / 2} e^{-x}$ as $x \rightarrow+\infty$ and bearing in mind the functional relation $\Gamma(x+1)=x \Gamma(x)$ for all $x>0$, we get two constants $\check{C}_{2}>0$ and $A_{2}>0$ independent of $j$ such that

$$
\begin{aligned}
\sup _{x \geq 0} & \frac{1+x^{2}}{x^{1 / \kappa}} e^{-v x} x G_{1}(x) \leq \check{C}_{2} A_{2}^{j}\left(\Gamma\left(\frac{h_{l}-h_{0}+j}{\kappa}\right)\right. \\
+ & \left.\Gamma\left(\frac{h_{l}-h_{0}+j}{\kappa}+2\right)\right) \\
& \leq \check{C}_{2} A_{2}^{j}\left(\Gamma\left(\frac{h_{l}-h_{0}+j}{\kappa}\right)+\left(\frac{h_{l}-h_{0}+j}{\kappa}+1\right)\right. \\
& \left.\cdot\left(\frac{h_{l}-h_{0}+j}{\kappa}\right) \Gamma\left(\frac{h_{l}-h_{0}+j}{\kappa}\right)\right) .
\end{aligned}
$$

On the other hand, by direct inspection, we observe that there exists a constant $\check{C}_{2.1}>0$ (independent of $j$ and $\epsilon$ ) such that

$$
\sup _{0 \leq x<1} \frac{1+x^{2}}{x^{1 / \kappa}} e^{-v x} \frac{x}{\left(1+|\epsilon|^{(\chi+\alpha) \kappa} x\right)^{\gamma_{1}}} G_{2}(x) \leq \check{C}_{2.1} .
$$

Furthermore, there exists a constant $K_{2.1}(j)$ depending on $\nu$, $\kappa, h_{l}$ for $0 \leq l \leq M$ and $j$, such that

$$
\begin{aligned}
& \sup _{x \geq 1} \frac{1+x^{2}}{x^{1 / \kappa}} e^{-\nu x} \frac{x}{\left(1+|\epsilon|^{(\chi+\alpha) \kappa} x\right)^{\gamma_{1}}} G_{2}(x) \\
& \quad \leq \sup _{x \geq 1} \frac{1+x^{2}}{1+(x / 2)^{2}} K_{2.1}(j) .
\end{aligned}
$$

Now, after a thorough examination of the proof of Proposition 2 out of [23], one can check that there exists a constant $\check{K}_{2.1}>0$ independent of $j$ such that

$$
K_{2.1}(j) \leq \check{K}_{2.1} \Gamma\left(\frac{h_{l}-h_{0}+j}{\kappa}\right)
$$

for all $j \geq 1$. Finally, gathering (120), (121), (122), and (123) yields the estimates (117).

Besides, we choose the radius $r_{\widetilde{Q}, \widetilde{R}_{D}}>0$ large enough and $\omega$ in such a manner that

$$
\begin{aligned}
\sum_{j=0}^{p} & \frac{C_{1}}{C_{\widetilde{P}}\left(r_{\widetilde{\mathrm{Q}}, \widetilde{R}_{D}}\right)^{1 / \delta_{D} \kappa} \Gamma\left(\left(b_{j}-k_{0}-\gamma\right) / \kappa\right)} \\
& \cdot \frac{\left\|\widetilde{B}_{j}(m)\right\|_{(\beta, \mu)}}{\inf _{m \in \mathbb{R}}\left|\widetilde{R}_{D}(i m)\right|}|\epsilon|^{n_{j}-\alpha b_{j}+\left(b_{j}-k_{0}-\gamma\right) \alpha}+\sum_{l=1}^{s}\left|a_{l}\right| \\
& \cdot \frac{C_{2}}{C_{\widetilde{P}}\left(r_{\widetilde{Q}, \widetilde{R}_{D}}\right)^{1 / \delta_{D} \kappa} \Gamma\left(\left(k_{l}-k_{0}\right) / \kappa\right)}|\epsilon|^{\alpha\left(k_{l}-k_{0}\right)} \omega \\
& +\sum_{l=s+1}^{q}\left|a_{l}\right|
\end{aligned}
$$




$$
\begin{aligned}
& \cdot \frac{C_{2}}{C_{\widetilde{P}}\left(r_{\widetilde{Q}, \widetilde{R}_{D}}\right)^{1 / \delta_{D} \kappa} \Gamma\left(\left(k_{l}-k_{0}\right) / \kappa\right)}|\epsilon|^{m_{l}+\beta-\alpha k_{l}+\alpha\left(k_{l}-k_{0}\right)} \\
& \cdot \varpi+2\left|\frac{a_{0}}{c_{0}}\right|\left(\sum_{l=1}^{s^{\prime}}\left|c_{l}\right|\right. \\
& \cdot \frac{C_{2}}{C_{\widetilde{P}}\left(r_{\widetilde{Q}, \tilde{R}_{D}}\right)^{1 / \delta_{D} \kappa} \Gamma\left(\left(h_{l}-h_{0}\right) / \kappa\right)}|\epsilon|^{\alpha\left(h_{l}-h_{0}\right)} \emptyset \\
& +\sum_{l=s^{\prime}+1}^{M}\left|c_{l}\right| \\
& \cdot \frac{C_{2}}{C_{\widetilde{P}}\left(r_{\widetilde{Q}, \widetilde{R}_{D}}\right)^{1 / \delta_{D} \kappa} \Gamma\left(\left(h_{l}-h_{0}\right) / \kappa\right)}|\epsilon|^{\mu_{l}+2 \beta-\alpha h_{l}} \\
& \left.\cdot|\epsilon|^{\alpha\left(h_{l}-h_{0}\right)} \emptyset\right)+2\left|\frac{a_{0}}{c_{0}}\right|\left(\sum_{l=0}^{s^{\prime}}\left|c_{l}\right|\right. \\
& \cdot \sum_{j \geq 1}\left|J_{j}\right| \frac{\widehat{C}_{2} A_{2}^{j}}{C_{\widetilde{P}}\left(r_{\widetilde{Q}, \widetilde{R}_{D}}\right)^{1 / \delta_{D} \kappa}}|\epsilon|^{\alpha\left(h_{l}-h_{0}+j\right)} \varpi+\sum_{l=s^{\prime}+1}^{M}\left|c_{l}\right| \\
& \left.\cdot \sum_{j \geq 1}\left|J_{j}\right| \frac{\widehat{C}_{2} A_{2}^{j}}{C_{\widetilde{P}}\left(r_{\widetilde{Q}, \widetilde{R}_{D}}\right)^{1 / \delta_{D} \kappa}}|\epsilon|^{\mu_{l}+2 \beta-\alpha h_{l}}|\epsilon|^{\alpha\left(h_{l}-h_{0}+j\right)} \emptyset\right) \\
& \leq \frac{\omega}{3} \text {. }
\end{aligned}
$$

Notice that the infinite sums over the integers $j \geq 0$ are convergent in the left-hand side of inequality (124), provided that $\epsilon_{0}>0$ is small enough, according to the fact that there exist two constants $J_{1}, J_{2}>0$ such that $\left|J_{j}\right| \leq J_{1}\left(J_{2}\right)^{j}$ for all $j \geq 1$ since $J(T)=\sum_{j \geq 1} J_{j} T^{j}$ is a convergent series near $T=0$.

From the definition of $\mathscr{H}_{\epsilon}^{1}$ given by (110), we deduce the following inequality:

$$
\left\|\mathscr{H}_{\epsilon}^{1}(w(\tau, m))\right\|_{(\nu, \beta, \mu, \chi, \alpha, \kappa, \epsilon)} \leq \frac{\Phi}{3} .
$$

Hereafter, we focus on norm estimates for each part of the map $\mathscr{H}_{\epsilon}^{2}$. We set

$$
\begin{gathered}
h(\tau, m)=\tau^{\kappa-1} \int_{0}^{\tau^{\kappa}} \int_{-\infty}^{+\infty} w\left(\left(\tau^{\kappa}-s^{\prime}\right)^{1 / \kappa}, m-m_{1}\right) \\
\cdot w\left(\left(s^{\prime}\right)^{1 / \kappa}, m_{1}\right) \frac{1}{\left(\tau^{\kappa}-s^{\prime}\right) s^{\prime}} d s^{\prime} d m_{1} .
\end{gathered}
$$

Regarding Proposition 6, we get a constant $C_{3}>0$ (depending on $\mu, \kappa$ ) such that

$$
\|h(\tau, m)\|_{(\nu, \beta, \mu, \chi, \alpha, \kappa, \epsilon)} \leq \frac{C_{3}}{|\epsilon|^{\chi+\alpha}}\|w(\tau, m)\|_{(\nu, \beta, \mu, \chi, \alpha, \kappa, \epsilon)}^{2} .
$$

On the other hand, using Proposition 5(2), we grab a constant $C_{2}^{\prime}>0$ (depending on $\nu, \kappa, \gamma, \delta_{D}, k_{0}, h_{l}$ for $0 \leq l \leq M$ and $\left.\widetilde{Q}(X), \widetilde{R}_{D}(X)\right)$ such that

$$
\begin{gathered}
\left\|\epsilon^{-\chi\left(-k_{0}+\gamma+h_{l}\right)} \frac{\widetilde{Q}(i m)}{\widetilde{P}_{m}(\tau)} \tau^{\kappa} \int_{0}^{\tau^{\kappa}}\left(\tau^{\kappa}-s\right)^{\left(-k_{0}+\gamma+h_{l}\right) / \kappa-1} s^{1 / \kappa-1} h\left(s^{1 / \kappa}, m\right) d s\right\|_{(\nu, \beta, \mu, \chi, \alpha, \kappa, \epsilon)} \\
\leq \frac{C_{2}^{\prime}}{C_{\widetilde{P}}\left(r_{\widetilde{Q}, \widetilde{R}_{D}}\right)^{1 / \delta_{D} \kappa}}|\epsilon|^{(\chi+\alpha) \kappa\left(\left(-k_{0}+\gamma+h_{l}\right) / \kappa+1 / \kappa\right)-\chi\left(-k_{0}+\gamma+h_{l}\right)-(\chi+\alpha) \kappa\left(\delta_{D}-1 / \kappa\right)}\|h(\tau, m)\|_{(\nu, \beta, \mu, \chi, \alpha, \kappa, \epsilon)} .
\end{gathered}
$$

Therefore, gathering (127) and (128) returns

$$
\begin{aligned}
& \| \epsilon^{-\chi\left(-k_{0}+\gamma+h_{l}\right)} \frac{\widetilde{Q}(i m)}{\widetilde{P}_{m}(\tau)} \tau^{\kappa} \int_{0}^{\tau^{\kappa}}\left(\tau^{\kappa}-s\right)^{\left(-k_{0}+\gamma+h_{l}\right) / \kappa-1} \\
& \quad \times\left\{s \int_{0}^{s} \int_{-\infty}^{+\infty} w\left(\left(s-s^{\prime}\right)^{1 / \kappa}, m-m_{1}\right) w\left(\left(s^{\prime}\right)^{1 / \kappa}, m_{1}\right) \frac{1}{\left(s-s^{\prime}\right) s^{\prime}} d s^{\prime} d m_{1}\right\} \frac{d s}{s} \|_{(\nu, \beta, \mu, \chi, \alpha, \kappa, \epsilon)} \\
& \quad \leq \frac{C_{2}^{\prime} C_{3}}{C_{\widetilde{P}}\left(r_{\widetilde{Q}, \widetilde{R}_{D}}\right)^{1 / \delta_{D} \kappa}}|\epsilon|^{(\chi+\alpha)\left(-k_{0}+\gamma+h_{l}-\kappa \delta_{D}+1\right)-\chi\left(-k_{0}+\gamma+h_{l}\right)}\|w(\tau, m)\|_{(\nu, \beta, \mu, \chi, \alpha, \kappa, \epsilon)}^{2} .
\end{aligned}
$$


Bearing in mind Proposition 5(1), we get a constant $C_{2}>0$ (depending on $v, \kappa, d_{l}, \delta_{l}$ and $\widetilde{R}_{l}(X), \widetilde{R}_{D}(X)$ for $1 \leq l \leq D-1$ ), such that

$$
\begin{gathered}
\| \epsilon^{-\chi\left(d_{l}-k_{0}-\delta_{l}\right)} \frac{\widetilde{R}_{l}(i m)}{\widetilde{P}_{m}(\tau)} \tau^{\kappa} \int_{0}^{\tau^{\kappa}}\left(\tau^{\kappa}-s\right)^{d_{l, \delta_{l}, 0} / \kappa-1} \\
\cdot w\left(s^{1 / \kappa}, m\right) \frac{d s}{s} \|_{(\nu, \beta, \mu, \chi, \alpha, \kappa, \epsilon)} \\
\leq \frac{C_{2}}{C_{\widetilde{P}}\left(r_{\widetilde{Q}, \widetilde{R}_{D}}\right)^{1 / \delta_{D} \kappa}}|\epsilon|^{(\chi+\alpha) d_{l, \delta_{l}, 0}-\chi\left(d_{l}-k_{0}-\delta_{l}\right)}\|w(\tau, m)\|_{(\nu, \beta, \mu, \chi, \alpha, \kappa, \epsilon)} \\
=\frac{C_{2}}{C_{\widetilde{P}}\left(r_{\widetilde{Q}, \widetilde{R}_{D}}\right)^{1 / \delta_{D} \kappa}|\epsilon|^{\alpha\left(d_{l}-k_{0}-\delta_{l}\right)}\|w(\tau, m)\|_{(\nu, \beta, \mu, \chi, \alpha, \kappa, \epsilon)} .}
\end{gathered}
$$

Likewise, we can apply Proposition 5(2) in order to exhibit a constant $C_{2}^{\prime}>0$ (depending on $\nu, \kappa, d_{l}, \delta_{l}, k_{0}, \delta_{D}$ and $\widetilde{R}_{l}(X)$, $\widetilde{R}_{D}(X)$ for $\left.1 \leq l \leq D-1\right)$ with

$$
\begin{gathered}
\| \epsilon^{-\chi\left(d_{l}-k_{0}-\delta_{l}\right)} \frac{\widetilde{R}_{l}(i m)}{\widetilde{P}_{m}(\tau)} \\
\cdot \tau^{\kappa} \int_{0}^{\tau^{\kappa}}\left(\tau^{\kappa}-s\right)^{d_{l, q_{1}, q_{2}} / \kappa-1} s^{q_{2}} w\left(s^{1 / \kappa}, m\right) \frac{d s}{s} \|_{(\nu, \beta, \mu, \chi, \alpha, \kappa, \epsilon)} \\
\leq \frac{C_{2}^{\prime}}{C_{\widetilde{P}}\left(r_{\widetilde{Q}, \widetilde{R}_{D}}\right)^{1 / \delta_{D} \kappa}}|\epsilon|^{(\chi+\alpha) \kappa\left(d_{l, q_{1}, q_{2}} / \kappa+q_{2}-\delta_{D}+1 / \kappa\right)-\chi\left(d_{l}-k_{0}-\delta_{l}\right)}
\end{gathered}
$$

$$
\cdot\|w(\tau, m)\|_{(\nu, \beta, \mu, \chi, \alpha, \kappa, \epsilon)}
$$

for all $q_{1} \geq 0$ and $q_{2} \geq 1$ with $q_{1}+q_{2}=\delta_{l}$. Besides,

$$
\begin{aligned}
& \| \epsilon^{-\chi\left(d_{l}-k_{0}-\delta_{l}\right)} \frac{\widetilde{R}_{l}(i m)}{\widetilde{P}_{m}(\tau)} \tau^{\kappa} \int_{0}^{\tau^{\kappa}}\left(\tau^{\kappa}-s\right)^{d_{l, q_{1}, q_{2}} / \kappa+q_{2}-p-1} \\
& \cdot s^{p} w\left(s^{1 / \kappa}, m\right) \frac{d s}{s} \|_{(\nu, \beta, \mu, \chi, \alpha, \kappa, \epsilon)} \\
& \leq \frac{C_{2}^{\prime}}{C_{\widetilde{P}}\left(r_{\widetilde{Q}, \widetilde{R}_{D}}\right)^{1 / \delta_{D} \kappa}}|\epsilon|^{(\chi+\alpha) \kappa\left(d_{l, q_{1}, q_{2}} / \kappa+q_{2}-\delta_{D}+1 / \kappa\right)-\chi\left(d_{l}-k_{0}-\delta_{l}\right)} \\
& \cdot\|w(\tau, m)\|_{(\nu, \beta, \mu, \chi, \alpha, \kappa, \epsilon)}
\end{aligned}
$$

provided that $q_{2} \geq 2$ and $1 \leq p \leq q_{2}-1$, with $q_{1}+q_{2}=\delta_{l}$. Now, we choose $r_{\widetilde{\mathrm{Q}}, \widetilde{R}_{D}}>0$ and $\omega$ in such a way that

$$
\begin{gathered}
\sum_{l=0}^{s^{\prime}}\left|c_{l}\right| \frac{C_{2}^{\prime} C_{3}}{C_{\widetilde{P}}\left(r_{\widetilde{Q}, \widetilde{R}_{D}}\right)^{1 / \delta_{D^{\kappa}}} \Gamma\left(\left(-k_{0}+\gamma+h_{l}\right) / \kappa\right)(2 \pi)^{1 / 2}} \\
\cdot|\epsilon|^{(\chi+\alpha)\left(-k_{0}+\gamma+h_{l}-\kappa \delta_{D}+1\right)-\chi\left(-k_{0}+\gamma+h_{l}\right)} \omega^{2}+\sum_{l=s^{\prime}+1}^{M}\left|c_{l}\right| \\
\cdot \frac{C_{2}^{\prime} C_{3}}{C_{\widetilde{P}}\left(r_{\widetilde{Q}, \widetilde{R}_{D}}\right)^{1 / \delta_{D} \kappa} \Gamma\left(\left(-k_{0}+\gamma+h_{l}\right) / \kappa\right)(2 \pi)^{1 / 2}}
\end{gathered}
$$

$$
\begin{aligned}
& \times|\epsilon|^{(\chi+\alpha)\left(-k_{0}+\gamma+h_{l}-\kappa \delta_{D}+1\right)-\chi\left(-k_{0}+\gamma+h_{l}\right)}|\epsilon|^{\mu_{l}+2 \beta-\alpha h_{l}} \omega^{2} \\
& +\sum_{l=1}^{D-1}|\epsilon|^{\Delta_{l}+\alpha\left(\delta_{l}-d_{l}\right)+\beta}\left[\prod_{d=0}^{\delta_{l}-1}|\gamma-d|\right. \\
& \cdot \frac{C_{2}}{C_{\widetilde{P}}\left(r_{\widetilde{Q}, \widetilde{R}_{D}}\right)^{1 / \delta_{D} \kappa} \Gamma\left(d_{l, \delta_{l}, 0} / \kappa\right)}|\epsilon|^{\alpha\left(d_{l}-k_{0}-\delta_{l}\right)} \omega \\
& +\sum_{q_{1}+q_{2}=\delta_{l}, q_{2} \geq 1} \frac{\delta_{l} !}{q_{1} ! q_{2} !} \prod_{d=0}^{q_{1}-1}|\gamma-d| \\
& \cdot \frac{C_{2}^{\prime} \kappa^{q_{2}}}{C_{\widetilde{P}}\left(r_{\widetilde{Q}, \widetilde{R}_{D}}\right)^{1 / \delta_{D} \kappa} \Gamma\left(d_{l, q_{1}, q_{2}} / \kappa\right)} \\
& \times|\epsilon|^{(\chi+\alpha) \kappa\left(d_{l, q_{1}, q_{2}} / \kappa+q_{2}-\delta_{D}+1 / \kappa\right)-\chi\left(d_{l}-k_{0}-\delta_{l}\right)} \omega \\
& +\sum_{1 \leq p \leq q_{2}-1}^{\left|A_{q_{2}, p}\right|} \\
& +\frac{C_{\widetilde{P}}\left(r_{\widetilde{Q}, \widetilde{R}_{D}}\right)^{1 / \delta_{D} \kappa} \Gamma\left(d_{l, q_{1}, q_{2}} / \kappa+q_{2}-p\right)}{C_{2}^{\prime} \kappa^{p}}
\end{aligned}
$$

With the help of the definition of $\mathscr{H}_{\epsilon}^{2}$ given by (111), we deduce that

$$
\left\|\mathscr{H}_{\epsilon}^{2}(w(\tau, m))\right\|_{(\nu, \beta, \mu, \chi, \alpha, \kappa, \epsilon)} \leq \frac{\omega}{3} .
$$

Ultimately, we direct our attention to norm estimates for $\mathscr{H}_{\epsilon}^{3}$.

Taking notice of Proposition 5(1), we get a constant $C_{2}>0$ (depending on $\nu, \kappa, k_{0}, \delta_{D}, d_{D}$ ), such that

$$
\begin{aligned}
& \| \epsilon^{-\chi\left(d_{D}-k_{0}-\delta_{D}\right)} \frac{\widetilde{R}_{D}(i m)}{\widetilde{P}_{m}(\tau)} \tau^{\kappa} \int_{0}^{\tau^{\kappa}}\left(\tau^{\kappa}-s\right)^{d_{D, \delta_{D}, 0} / \kappa-1} \\
& \cdot w\left(s^{1 / \kappa}, m\right) \frac{d s}{s} \|_{(\nu, \beta, \mu, \chi, \alpha, \kappa, \epsilon)} \\
& \leq \frac{C_{2}}{C_{\widetilde{P}}\left(r_{\widetilde{Q}, \widetilde{R}_{D}}\right)^{1 / \delta_{D} \kappa}}|\epsilon|^{(\chi+\alpha) d_{D, \delta_{D}, 0}-\chi\left(d_{D}-k_{0}-\delta_{D}\right)} \\
& \cdot\|w(\tau, m)\|_{(\nu, \beta, \mu, \chi, \alpha, \kappa, \epsilon)} \\
& =\frac{C_{2}}{C_{\widetilde{P}}\left(r_{\widetilde{Q}, \widetilde{R}_{D}}\right)^{1 / \delta_{D} \kappa}|\epsilon|^{\alpha\left(d_{D}-k_{0}-\delta_{D}\right)}\|w(\tau, m)\|_{(\nu, \beta, \mu, \chi, \alpha, \kappa, \epsilon)} .}
\end{aligned}
$$


Moreover, we can apply Proposition 5(2) in order to exhibit a constant $C_{2}^{\prime}>0$ (depending on $v, \kappa, k_{0}, d_{D}$, and $\delta_{D}$ ) with

$$
\begin{aligned}
& \| \epsilon^{-\chi\left(d_{D}-k_{0}-\delta_{D}\right)} \frac{\widetilde{R}_{D}(i m)}{\widetilde{P}_{m}(\tau)} \\
& \cdot \tau^{\kappa} \int_{0}^{\tau^{\kappa}}\left(\tau^{\kappa}-s\right)^{d_{D, q_{1}, q_{2}} / \kappa-1} s^{q_{2}} w\left(s^{1 / \kappa}, m\right) \frac{d s}{s} \|_{(\nu, \beta, \mu, \gamma, \alpha, \kappa, \epsilon)} \\
& \leq \frac{C_{2}^{\prime}}{C_{\widetilde{P}}\left(r_{\widetilde{Q}} \widetilde{R}_{D}\right)^{1 / \delta_{D} \kappa}}|\epsilon|^{(\chi+\alpha) \kappa\left(d_{D, q_{1}, q_{2}} / \kappa+q_{2}-\delta_{D}+1 / \kappa\right)-\chi\left(d_{D}-k_{0}-\delta_{D}\right)} \\
& \cdot\|w(\tau, m)\|_{(\nu, \beta, \mu, \gamma, \alpha, \kappa, \epsilon)}
\end{aligned}
$$

for all $q_{1} \geq 1$ and $q_{2} \geq 1$ with $q_{1}+q_{2}=\delta_{D}$. Besides,

$$
\begin{aligned}
& \| \epsilon^{-\chi\left(d_{D}-k_{0}-\delta_{D}\right)} \frac{\widetilde{R}_{D}(i m)}{\widetilde{P}_{m}(\tau)} \tau^{\kappa} \int_{0}^{\tau^{\kappa}}\left(\tau^{\kappa}-s\right)^{d_{D, q_{1}, q_{2}} / \kappa+q_{2}-p-1} \\
& \quad s^{p} w\left(s^{1 / \kappa}, m\right) \frac{d s}{s} \|_{(\nu, \beta, \mu, \chi, \alpha, \alpha, \epsilon)} \\
& \leq \frac{C_{2}^{\prime}}{C_{\widetilde{P}}\left(r_{\widetilde{Q}, \tilde{R}_{D}}\right)^{1 / \delta_{D} \kappa}}|\epsilon|^{(\chi+\alpha) \kappa\left(d_{D, q_{1}, q_{2}} / \kappa+q_{2}-\delta_{D}+1 / \kappa\right)-\chi\left(d_{D}-k_{0}-\delta_{D}\right)} \\
& \cdot\|w(\tau, m)\|_{(\nu, \beta, \mu, \gamma, \alpha, \kappa, \epsilon)}
\end{aligned}
$$

provided that $q_{1} \geq 1, q_{2} \geq 2$, and $1 \leq p \leq q_{2}-1$ with $q_{1}+q_{2}=$ $\delta_{D}$. Finally, we can select a constant $C_{2}^{\prime}>0$ (depending on $v$, $\kappa$, and $\delta_{D}$ ) such that

$$
\begin{gathered}
\left\|\frac{\widetilde{R}_{D}(i m)}{\widetilde{P}_{m}(\tau)} \tau^{\kappa} \int_{0}^{\tau^{\kappa}}\left(\tau^{\kappa}-s\right)^{\delta_{D}-p-1} s^{p} w\left(s^{1 / \kappa}, m\right) \frac{d s}{s}\right\|_{(\nu, \beta, \mu, \gamma, \alpha, \alpha, \epsilon)} \\
\quad \leq \frac{C_{2}^{\prime}}{C_{\widetilde{P}}\left(r_{\widetilde{Q}, \widetilde{R}_{D}}\right)^{1 / \delta_{D} \kappa}}|\epsilon|^{\chi+\alpha}\|w(\tau, m)\|_{(\nu, \beta, \mu, \chi, \alpha, \kappa, \epsilon)}
\end{gathered}
$$

for all $1 \leq p \leq \delta_{D}-1$. We make the choice for the size of radius $r_{\widetilde{Q}, \widetilde{R}_{D}}$ and $\Phi$ in such a manner that

$$
\begin{aligned}
& |\epsilon|^{\Delta_{D}+\alpha\left(\delta_{D}-d_{D}\right)+\beta}\left[\prod_{d=0}^{\delta_{D}-1}|\gamma-d|\right. \\
& \cdot \frac{C_{2}}{C_{\widetilde{P}}\left(r_{\widetilde{Q}, \widetilde{R}_{D}}\right)^{1 / \delta_{D} \kappa} \Gamma\left(d_{D, \delta_{D}, 0} / \kappa\right)}|\epsilon|^{\alpha\left(d_{D}-k_{0}-\delta_{D}\right)} \emptyset \\
& +\sum_{q_{1}+q_{2}=\delta_{D}, q_{1} \geq 1, q_{2} \geq 1} \frac{\delta_{D} !}{q_{1} ! q_{2} !} \prod_{d=0}^{q_{1}-1}|\gamma-d| \\
& \cdot\left(\frac{C_{2}^{\prime} \kappa^{q_{2}}}{C_{\widetilde{P}}\left(r_{\widetilde{Q}, \widetilde{R}_{D}}\right)^{1 / \delta_{D} \kappa} \Gamma\left(d_{D, q_{1}, q_{2}} / \kappa\right)}\right. \\
& \times|\epsilon|^{(\chi+\alpha) \kappa\left(d_{D, q_{1}, q_{2}} / \kappa+q_{2}-\delta_{D}+1 / \kappa\right)-\chi\left(d_{D}-k_{0}-\delta_{D}\right)} \omega \\
& +\sum_{1 \leq p \leq q_{2}-1}\left|A_{q_{2}, p}\right|
\end{aligned}
$$

$$
\begin{aligned}
& \cdot \frac{C_{2}^{\prime} \kappa^{p}}{C_{\widetilde{P}}\left(r_{\widetilde{Q}, \widetilde{R}_{D}}\right)^{1 / \delta_{D} \kappa} \Gamma\left(d_{D, q_{1}, q_{2}} / \kappa+q_{2}-p\right)} \\
& \left.\left.\times|\epsilon|^{(\chi+\alpha) \kappa\left(d_{D, q_{1}, q_{2}} / \kappa+q_{2}-\delta_{D}+1 / \kappa\right)-\chi\left(d_{D}-k_{0}-\delta_{D}\right)} \varpi\right)\right] \\
& +\sum_{1 \leq p \leq \delta_{D}-1}\left|A_{\delta_{D}, p}\right| \\
& \cdot \frac{C_{2}^{\prime} \kappa^{p}}{C_{\widetilde{P}}\left(r_{\widetilde{Q}, \tilde{R}_{D}}\right)^{1 / \delta_{D} \kappa} \Gamma\left(\delta_{D}-p\right)}|\epsilon|^{\chi+\alpha} \circlearrowleft \leq \frac{\omega}{3} .
\end{aligned}
$$

From the construction of the map $\mathscr{H}_{\epsilon}^{3}$, it is now clear that

$$
\left\|\mathscr{H}_{\epsilon}^{3}(w(\tau, m))\right\|_{(\nu, \beta, \mu, \chi, \alpha, \kappa, \epsilon)} \leq \frac{\omega}{3} .
$$

Eventually, gathering (125), (134), and (140) yields the first claim (113).

In the last part of the proof, we fix our attention to the affirmation (114). Let $w_{1}(\tau, m), w_{2}(\tau, m) \in F_{(\nu, \beta, \mu, \chi, \alpha, \kappa, \epsilon)}^{d}$ with

$$
\begin{gathered}
\left\|w_{1}(\tau, m)\right\|_{(\nu, \beta, \mu, \chi, \alpha, \kappa, \epsilon)} \leq \omega, \\
\left\|w_{2}(\tau, m)\right\|_{(\nu, \beta, \mu, \chi, \alpha, \kappa, \epsilon)} \leq \omega .
\end{gathered}
$$

We first prove that $\mathscr{H}_{\epsilon}^{1}$ is a shrinking map. According to estimates (116) we obtain a constant $C_{2}>0$ (depending on $\nu, \kappa, k_{l}$, for $0 \leq l \leq q, h_{l}$, for $\left.0 \leq l \leq M, \widetilde{Q}(X), \widetilde{R}_{D}(X)\right)$ and a constant $C_{2}(j)$ satisfying the estimates (117) such that

$$
\begin{aligned}
& \| e^{-\chi\left(k_{1}-k_{0}\right)} \frac{\widetilde{Q}(i m) \tau^{\kappa}}{\widetilde{P}_{m}(\tau)} \int_{0}^{\tau^{\kappa}}\left(\tau^{\kappa}-s\right)^{\left(k_{l}-k_{0}\right) / \kappa-1} \\
& \cdot\left(w_{1}\left(s^{1 / \kappa}, m\right)-w_{2}\left(s^{1 / \kappa}, m\right)\right) \frac{d s}{s} \|_{(\nu, \beta, \mu, \gamma, \alpha, \alpha, \epsilon)} \\
& \leq \frac{C_{2}}{C_{\widetilde{P}}\left(r_{\widetilde{Q}, \tilde{R}_{D}}\right)^{1 / \delta_{D} \kappa}}|\epsilon|^{\alpha\left(k_{l}-k_{0}\right)}\left\|w_{1}(\tau, m)-w_{2}(\tau, m)\right\|_{(\nu, \beta, \mu, \mu, \alpha, \alpha, \epsilon)}, \\
& \| \epsilon^{-\chi\left(h_{l}-h_{0}\right)} \frac{\widetilde{Q}(i m) \tau^{\kappa}}{\widetilde{P}_{m}(\tau)} \int_{0}^{\tau^{\kappa}}\left(\tau^{\kappa}-s\right)^{\left(h_{l}-h_{0}\right) / \kappa-1} \\
& \cdot\left(w_{1}\left(s^{1 / \kappa}, m\right)-w_{2}\left(s^{1 / \kappa}, m\right)\right) \frac{d s}{s} \|_{(\nu, \beta, \mu, \gamma, \alpha, \alpha, \epsilon)} \\
& \leq \frac{C_{2}}{C_{\widetilde{P}}\left(r_{\widetilde{Q}, \tilde{P}_{D}}\right)^{1 / \delta_{D} \kappa}}|\epsilon|^{\alpha\left(h_{l}-h_{0}\right)}\left\|w_{1}(\tau, m)-w_{2}(\tau, m)\right\|_{(\nu, \beta, \mu, \mu, \alpha, \alpha, \epsilon)},
\end{aligned}
$$

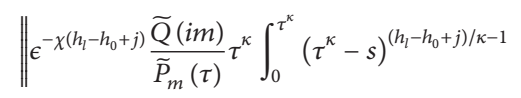

$$
\begin{aligned}
& \cdot\left(w_{1}\left(s^{1 / \kappa}, m\right)-w_{2}\left(s^{1 / \kappa}, m\right)\right) \frac{d s}{s} \|_{(\nu, \beta, \mu, \chi, \alpha, \alpha, \epsilon)} \\
& \leq \frac{C_{2}(j)}{C_{\widetilde{P}}\left(r_{\widetilde{Q}, \tilde{R}_{D}}\right)^{1 / \delta_{D} \kappa}}|\epsilon|^{\alpha\left(h_{1}-h_{0}+j\right)} \| w_{1}(\tau, m) \\
& -w_{2}(\tau, m) \|_{(\nu, \beta, \mu, \gamma, \alpha, \alpha, \epsilon)} \text {. }
\end{aligned}
$$


Therefore, we choose the radius $r_{\widetilde{Q}^{,} \widetilde{R}_{D}}>0$ large enough in order that

$$
\begin{aligned}
& \sum_{l=1}^{s}\left|a_{l}\right| \frac{C_{2}}{C_{\widetilde{P}}\left(r_{\widetilde{Q}, \widetilde{R}_{D}}\right)^{1 / \delta_{D} \kappa} \Gamma\left(\left(k_{l}-k_{0}\right) / \kappa\right)}|\epsilon|^{\alpha\left(k_{l}-k_{0}\right)} \\
& +\sum_{l=s+1}^{q}\left|a_{l}\right| \\
& \cdot \frac{C_{2}}{C_{\widetilde{P}}\left(r_{\widetilde{Q}, \widetilde{R}_{D}}\right)^{1 / \delta_{D} \kappa} \Gamma\left(\left(k_{l}-k_{0}\right) / \kappa\right)}|\epsilon|^{m_{l}+\beta-\alpha k_{l}+\alpha\left(k_{l}-k_{0}\right)} \\
& +2\left|\frac{a_{0}}{c_{0}}\right|\left(\sum_{l=1}^{s^{\prime}}\left|c_{l}\right|\right. \\
& \cdot \frac{C_{2}}{C_{\widetilde{P}}\left(r_{\widetilde{Q}, \widetilde{R}_{D}}\right)^{1 / \delta_{D} \kappa} \Gamma\left(\left(h_{l}-h_{0}\right) / \kappa\right)}|\epsilon|^{\alpha\left(h_{l}-h_{0}\right)} \\
& +\sum_{l=s^{\prime}+1}^{M}\left|c_{l}\right| \\
& \cdot \frac{C_{2}}{C_{\widetilde{P}}\left(r_{\widetilde{Q}, \mathbb{R}_{D}}\right)^{1 / \delta_{D} \kappa} \Gamma\left(\left(h_{l}-h_{0}\right) / \kappa\right)}|\epsilon|^{\mu_{l}+2 \beta-\alpha h_{l}} \\
& \left.\cdot|\epsilon|^{\alpha\left(h_{l}-h_{0}\right)}\right)+2\left|\frac{a_{0}}{c_{0}}\right|\left(\sum_{l=0}^{s^{\prime}}\left|c_{l}\right|\right. \\
& \cdot \sum_{j \geq 1}\left|J_{j}\right| \frac{\widehat{C}_{2} A_{2}^{j}}{C_{\widetilde{P}}\left(r_{\widetilde{\mathrm{Q}}, \widetilde{R}_{D}}\right)^{1 / \delta_{D} \kappa}}|\epsilon|^{\alpha\left(h_{l}-h_{0}+j\right)}+\sum_{l=s^{\prime}+1}^{M}\left|c_{l}\right| \\
& \left.\cdot \sum_{j \geq 1}\left|J_{j}\right| \frac{\widehat{C}_{2} A_{2}^{j}}{C_{\widetilde{P}}\left(r_{\widetilde{Q}, \widetilde{R}_{D}}\right)^{1 / \delta_{D} \kappa}}|\epsilon|^{\mu_{l}+2 \beta-\alpha h_{l}}|\epsilon|^{\alpha\left(h_{l}-h_{0}+j\right)}\right) \\
& \leq \frac{1}{6} \text {. }
\end{aligned}
$$

As a result, we can set down

$$
\begin{gathered}
\left\|\mathscr{H}_{\epsilon}^{1}\left(w_{1}(\tau, m)\right)-\mathscr{H}_{\epsilon}^{1}\left(w_{2}(\tau, m)\right)\right\|_{(\nu, \beta, \mu, \chi, \alpha, \kappa, \epsilon)} \\
\leq \frac{1}{6}\left\|w_{1}(\tau, m)-w_{2}(\tau, m)\right\|_{(\nu, \beta, \mu, \chi, \alpha, \kappa, \epsilon)} .
\end{gathered}
$$

We turn to $\mathscr{H}_{\epsilon}^{2}$ and show that it is a shrinking map as well. As a preparation, we may first rewrite

$$
\begin{aligned}
& w_{1}\left(\left(\tau^{\kappa}-s^{\prime}\right)^{1 / \kappa}, m-m_{1}\right) w_{1}\left(\left(s^{\prime}\right)^{1 / \kappa}, m_{1}\right) \\
& -w_{2}\left(\left(\tau^{\kappa}-s^{\prime}\right)^{1 / \kappa}, m-m_{1}\right) w_{2}\left(\left(s^{\prime}\right)^{1 / \kappa}, m_{1}\right) \\
& =\left(w_{1}\left(\left(\tau^{\kappa}-s^{\prime}\right)^{1 / \kappa}, m-m_{1}\right)\right. \\
& \left.-w_{2}\left(\left(\tau^{\kappa}-s^{\prime}\right)^{1 / \kappa}, m-m_{1}\right)\right) w_{1}\left(\left(s^{\prime}\right)^{1 / \kappa}, m_{1}\right) \\
& +w_{2}\left(\left(\tau^{\kappa}-s^{\prime}\right)^{1 / \kappa}, m-m_{1}\right)\left(w_{1}\left(\left(s^{\prime}\right)^{1 / \kappa}, m_{1}\right)\right. \\
& \left.-w_{2}\left(\left(s^{\prime}\right)^{1 / \kappa}, m_{1}\right)\right) .
\end{aligned}
$$

For $j=1,2$, we set

$$
\begin{gathered}
h_{j}(\tau, m)=\tau^{\kappa-1} \int_{0}^{\tau^{\kappa}} \int_{-\infty}^{+\infty} w_{j}\left(\left(\tau^{\kappa}-s^{\prime}\right)^{1 / \kappa}, m-m_{1}\right) \\
\cdot w_{j}\left(\left(s^{\prime}\right)^{1 / \kappa}, m_{1}\right) \frac{1}{\left(\tau^{\kappa}-s^{\prime}\right) s^{\prime}} d s^{\prime} d m_{1} .
\end{gathered}
$$

Regarding both the factorization (145) above and Proposition 6 , we get a constant $C_{3}>0$ (depending on $\left.\mu, \kappa\right)$ such that

$$
\begin{aligned}
& \left\|h_{1}(\tau, m)-h_{2}(\tau, m)\right\|_{(\nu, \beta, \mu, \chi, \alpha, \kappa, \epsilon)} \\
& \quad \leq \frac{C_{3}}{|\epsilon|^{\chi+\alpha}}\left(\left\|w_{1}(\tau, m)\right\|_{(\nu, \beta, \mu, \chi, \alpha, \kappa, \epsilon)}\right. \\
& \left.\quad+\left\|w_{2}(\tau, m)\right\|_{(\nu, \beta, \mu, \chi, \alpha, \kappa, \epsilon)}\right) \times \| w_{1}(\tau, m) \\
& \quad-w_{2}(\tau, m) \|_{(\nu, \beta, \mu, \chi, \alpha, \kappa, \epsilon)} .
\end{aligned}
$$

From (128) together with (147) we pick up a constant $C_{2}^{\prime}>0$ (depending on $\nu, \kappa, \gamma, \delta_{D}, k_{0}, h_{l}$ for $0 \leq l \leq M$ and $\widetilde{Q}(X)$, $\left.\widetilde{R}_{D}(X)\right)$ such that

$$
\begin{aligned}
& \left\|\epsilon^{-\chi\left(-k_{0}+\gamma+h_{l}\right)} \frac{\widetilde{Q}(i m)}{\widetilde{P}_{m}(\tau)} \tau^{\kappa} \int_{0}^{\tau^{\kappa}}\left(\tau^{\kappa}-s\right)^{\left(-k_{0}+\gamma+h_{l}\right) / \kappa-1} s^{1 / \kappa-1}\left(h_{1}\left(s^{1 / \kappa}, m\right)-h_{2}\left(s^{1 / \kappa}, m\right)\right) d s\right\|_{(\nu, \beta, \mu, \chi, \alpha, \kappa, \epsilon)} \\
& \leq \frac{C_{2}^{\prime}}{C_{\widetilde{P}}\left(r_{\widetilde{Q}, \widetilde{R}_{D}}\right)^{1 / \delta_{D} \kappa}}|\epsilon|^{(\chi+\alpha) \kappa\left(\left(-k_{0}+\gamma+h_{l}\right) / \kappa+1 / \kappa\right)-\chi\left(-k_{0}+\gamma+h_{l}\right)-(\chi+\alpha) \kappa\left(\delta_{D}-1 / \kappa\right)}\left\|h_{1}(\tau, m)-h_{2}(\tau, m)\right\|_{(\nu, \beta, \mu, \chi, \alpha, \kappa, \epsilon)} \\
& \leq \frac{C_{2}^{\prime} C_{3}}{C_{\widetilde{P}}\left(r_{\widetilde{Q}, \widetilde{R}_{D}}\right)^{1 / \delta_{D} \kappa}}|\epsilon|^{(\chi+\alpha)\left(-k_{0}+\gamma+h_{l}-\kappa \delta_{D}+1\right)-\chi\left(-k_{0}+\gamma+h_{l}\right)} \\
& \quad \times\left(\left\|w_{1}(\tau, m)\right\|_{(\nu, \beta, \mu, \chi, \alpha, \kappa, \epsilon)}+\left\|w_{2}(\tau, m)\right\|_{(\nu, \beta, \mu, \chi, \alpha, \kappa, \epsilon)}\right)\left\|w_{1}(\tau, m)-w_{2}(\tau, m)\right\|_{(\nu, \beta, \mu, \chi, \alpha, \kappa, \epsilon)} .
\end{aligned}
$$


Bearing in mind (130), we get a constant $C_{2}>0$ (depending on $\nu, \kappa, d_{l}, \delta_{l}$ and $\widetilde{R}_{l}(X), \widetilde{R}_{D}(X)$ for $\left.1 \leq l \leq D-1\right)$, such that

$$
\begin{aligned}
& \| \epsilon^{-\chi\left(d_{l}-k_{0}-\delta_{l}\right)} \frac{\widetilde{R}_{l}(i m)}{\widetilde{P}_{m}(\tau)} \tau^{\kappa} \int_{0}^{\tau^{\kappa}}\left(\tau^{\kappa}-s\right)^{d_{l, \delta_{l}, 0} / \kappa-1} \\
& \cdot\left(w_{1}\left(s^{1 / \kappa}, m\right)-w_{2}\left(s^{1 / \kappa}, m\right)\right) \frac{d s}{s} \|_{(\nu, \beta, \mu, \chi, \alpha, \kappa, \epsilon)} \\
& \leq \frac{C_{2}}{C_{\widetilde{P}}\left(r_{\widetilde{Q}, \widetilde{R}_{D}}\right)^{1 / \delta_{D} \kappa}}|\epsilon|^{\alpha\left(d_{l}-k_{0}-\delta_{l}\right)} \| w_{1}(\tau, m) \\
& -w_{2}(\tau, m) \|_{(\nu, \beta, \mu, \chi, \alpha, \kappa, \epsilon)} .
\end{aligned}
$$

Likewise, we can apply (131) in order to exhibit a constant $C_{2}^{\prime}>0$ (depending on $\nu, \kappa, d_{l}, \delta_{l}, k_{0}, \delta_{D}$ and $\widetilde{R}_{l}(X), \widetilde{R}_{D}(X)$ for $1 \leq l \leq D-1)$ with

$$
\begin{aligned}
& \| \epsilon^{-\chi\left(d_{l}-k_{0}-\delta_{l}\right)} \frac{\widetilde{R}_{l}(i m)}{\widetilde{P}_{m}(\tau)} \tau^{\kappa} \int_{0}^{\tau^{\kappa}}\left(\tau^{\kappa}-s\right)^{d_{l, q_{1}, q_{2}} / \kappa-1} \\
& \cdot s^{q_{2}}\left(w_{1}\left(s^{1 / \kappa}, m\right)-w_{2}\left(s^{1 / \kappa}, m\right)\right) \frac{d s}{s} \|_{(\nu, \beta, \mu, \chi, \alpha, \kappa, \epsilon)}
\end{aligned}
$$

$$
\begin{aligned}
& \leq \frac{C_{2}^{\prime}}{C_{\widetilde{P}}\left(r_{\widetilde{Q}, \widetilde{R}_{D}}\right)^{1 / \delta_{D} \kappa}}|\epsilon|^{(\chi+\alpha) \kappa\left(d_{l, q_{1}, q_{2}} / \kappa+q_{2}-\delta_{D}+1 / \kappa\right)-\chi\left(d_{l}-k_{0}-\delta_{l}\right)} \\
& \cdot\left\|w_{1}(\tau, m)-w_{2}(\tau, m)\right\|_{(\nu, \beta, \mu, \chi, \alpha, \kappa, \epsilon)}
\end{aligned}
$$

for all $q_{1} \geq 0$ and $q_{2} \geq 1$ with $q_{1}+q_{2}=\delta_{l}$. Furthermore, from (132) we deduce

$$
\begin{aligned}
& \| \epsilon^{-\chi\left(d_{l}-k_{0}-\delta_{l}\right)} \frac{\widetilde{R}_{l}(i m)}{\widetilde{P}_{m}(\tau)} \tau^{\kappa} \int_{0}^{\tau^{\kappa}}\left(\tau^{\kappa}-s\right)^{d_{l, q_{1}, q_{2}} / \kappa+q_{2}-p-1} \\
& \cdot s^{p}\left(w_{1}\left(s^{1 / \kappa}, m\right)-w_{2}\left(s^{1 / \kappa}, m\right)\right) \frac{d s}{s} \|_{(\nu, \beta, \mu, \chi, \alpha, \kappa, \epsilon)} \\
& \leq \frac{C_{2}^{\prime}}{C_{\widetilde{P}}\left(r_{\widetilde{Q}, \widetilde{R}_{D}}\right)^{1 / \delta_{D} \kappa}}|\epsilon|^{(\chi+\alpha) \kappa\left(d_{h q_{1}, q_{2}} / \kappa+q_{2}-\delta_{D}+1 / \kappa\right)-\chi\left(d_{l}-k_{0}-\delta_{l}\right)} \\
& \cdot\left\|w_{1}(\tau, m)-w_{2}(\tau, m)\right\|_{(\nu, \beta, \mu, \chi, \alpha, \alpha, \kappa)}
\end{aligned}
$$

provided that $q_{2} \geq 2$ and $1 \leq p \leq q_{2}-1$, with $q_{1}+q_{2}=\delta_{l}$.

Now, we sort $r_{\widetilde{\mathrm{Q}}, \widetilde{R}_{D}}>0$ and $\omega$ in such a way that

$$
\begin{aligned}
& \sum_{l=0}^{s^{\prime}}\left|c_{l}\right| \frac{C_{2}^{\prime} C_{3}}{C_{\widetilde{P}}\left(r_{\widetilde{Q}, \widetilde{R}_{D}}\right)^{1 / \delta_{D} \kappa} \Gamma\left(\left(-k_{0}+\gamma+h_{l}\right) / \kappa\right)(2 \pi)^{1 / 2}}|\epsilon|^{(\chi+\alpha)\left(-k_{0}+\gamma+h_{l}-\kappa \delta_{D}+1\right)-\chi\left(-k_{0}+\gamma+h_{l}\right)} \times\left(\left\|w_{1}(\tau, m)\right\|_{(\nu, \beta, \mu, \chi, \alpha, \kappa, \epsilon)}\right. \\
& \left.+\left\|w_{2}(\tau, m)\right\|_{(\nu, \beta, \mu, \chi, \alpha, \kappa, \epsilon)}\right)+\sum_{l=s^{\prime}+1}^{M}\left|c_{l}\right| \frac{C_{2}^{\prime} C_{3}}{C_{\widetilde{P}}\left(r_{\widetilde{Q}, \widetilde{R}_{D}}\right)^{1 / \delta_{D} \kappa} \Gamma\left(\left(-k_{0}+\gamma+h_{l}\right) / \kappa\right)(2 \pi)^{1 / 2}}|\epsilon|^{(\chi+\alpha)\left(-k_{0}+\gamma+h_{l}-\kappa \delta_{D}+1\right)-\chi\left(-k_{0}+\gamma+h_{l}\right)} \\
& \times|\epsilon|^{\mu_{l}+2 \beta-\alpha h_{l}}\left(\left\|w_{1}(\tau, m)\right\|_{(\nu, \beta, \mu, \chi, \alpha, \kappa, \epsilon)}+\left\|w_{2}(\tau, m)\right\|_{(\nu, \beta, \mu, \chi, \alpha, \kappa, \epsilon)}\right)+\sum_{l=1}^{D-1}|\epsilon|^{\Delta_{l}+\alpha\left(\delta_{l}-d_{l}\right)+\beta}\left[\prod_{d=0}^{\delta_{l}-1}|\gamma-d|\right. \\
& \cdot \frac{C_{2}}{C_{\widetilde{P}}\left(r_{\widetilde{Q}, \widetilde{R}_{D}}\right)^{1 / \delta_{D} \kappa} \Gamma\left(d_{l, \delta_{l}, 0} / \kappa\right)}|\epsilon|^{\alpha\left(d_{l}-k_{0}-\delta_{l}\right)}+\sum_{q_{1}+q_{2}=\delta_{l}, q_{2} \geq 1} \frac{\delta_{l} !}{q_{1} ! q_{2} !} \prod_{d=0}^{q_{1}-1}|\gamma-d|\left(\frac{C_{2}^{\prime} \kappa^{q_{2}}}{C_{\widetilde{P}}\left(r_{\widetilde{Q}, \widetilde{R}_{D}}\right)^{1 / \delta_{D} \kappa} \Gamma\left(d_{l, q_{1}, q_{2}} / \kappa\right)}\right. \\
& \times|\epsilon|^{(\chi+\alpha) \kappa\left(d_{l, q_{1}, q_{2}} / \kappa+q_{2}-\delta_{D}+1 / \kappa\right)-\chi\left(d_{l}-k_{0}-\delta_{l}\right)}+\sum_{1 \leq p \leq q_{2}-1}\left|A_{q_{2}, p}\right| \frac{C_{2}^{\prime} \kappa^{p}}{C_{\widetilde{P}}\left(r_{\widetilde{Q}, \widetilde{R}_{D}}\right)^{1 / \delta_{D} \kappa} \Gamma\left(d_{l, q_{1}, q_{2}} / \kappa+q_{2}-p\right)} \\
& \left.\left.\times|\epsilon|^{(\chi+\alpha) \kappa\left(d_{h q_{1}, q_{2}} / \kappa+q_{2}-\delta_{D}+1 / \kappa\right)-\chi\left(d_{l}-k_{0}-\delta_{l}\right)}\right)\right] \leq \frac{1}{6} .
\end{aligned}
$$

Subsequently, we obtain

$$
\begin{gathered}
\left\|\mathscr{H}_{\epsilon}^{2}\left(w_{1}(\tau, m)\right)-\mathscr{H}_{\epsilon}^{2}\left(w_{2}(\tau, m)\right)\right\|_{(\nu, \beta, \mu, \chi, \alpha, \kappa, \epsilon)} \\
\quad \leq \frac{1}{6}\left\|w_{1}(\tau, m)-w_{2}(\tau, m)\right\|_{(\nu, \beta, \mu, \chi, \alpha, \kappa, \epsilon)} .
\end{gathered}
$$

The last operation will be devoted to the proof that $\mathscr{H}_{\epsilon}^{3}$ is a shrinking map.
Taking notice of (135), we get a constant $C_{2}>0$ (depending on $v, \kappa, k_{0}, \delta_{D}$, and $d_{D}$ ), such that

$$
\begin{gathered}
\| \epsilon^{-\chi\left(d_{D}-k_{0}-\delta_{D}\right)} \frac{\widetilde{R}_{D}(i m)}{\widetilde{P}_{m}(\tau)} \tau^{\kappa} \int_{0}^{\tau^{\kappa}}\left(\tau^{\kappa}-s\right)^{d_{D, \delta_{D}, 0} / \kappa-1} \\
\cdot\left(w_{1}\left(s^{1 / \kappa}, m\right)-w_{2}\left(s^{1 / \kappa}, m\right)\right) \frac{d s}{s} \|_{(\nu, \beta, \mu, \chi, \alpha, \kappa, \epsilon)}
\end{gathered}
$$




$$
\begin{aligned}
& \leq \frac{C_{2}}{C_{\widetilde{P}}\left(r_{\widetilde{Q}, \widetilde{R}_{D}}\right)^{1 / \delta_{D} \kappa}}|\epsilon|^{\alpha\left(d_{D}-k_{0}-\delta_{D}\right)} \| w_{1}(\tau, m) \\
& -w_{2}(\tau, m) \|_{(\nu, \beta, \mu, \chi, \alpha, \kappa, \epsilon)} .
\end{aligned}
$$

Moreover, we may have a look at (136) in order to exhibit a constant $C_{2}^{\prime}>0$ (depending on $v, \kappa, k_{0}, d_{D}$, and $\delta_{D}$ ) with

$$
\begin{gathered}
\| \epsilon^{-\chi\left(d_{D}-k_{0}-\delta_{D}\right)} \frac{\widetilde{R}_{D}(i m)}{\widetilde{P}_{m}(\tau)} \tau^{\kappa} \times \int_{0}^{\tau^{\kappa}}\left(\tau^{\kappa}-s\right)^{d_{D, q_{1}, q_{2}} / \kappa-1} \\
\cdot s^{q_{2}}\left(w_{1}\left(s^{1 / \kappa}, m\right)-w_{2}\left(s^{1 / \kappa}, m\right)\right) \frac{d s}{s} \|_{(\nu, \beta, \mu, \chi, \alpha, \kappa, \epsilon)} \\
\leq \frac{C_{2}^{\prime}}{C_{\widetilde{P}}\left(r_{\widetilde{Q}, \widetilde{R}_{D}}\right)^{1 / \delta_{D} \kappa}}|\epsilon|^{(\chi+\alpha) \kappa\left(d_{D, q_{1}, q_{2}} / \kappa+q_{2}-\delta_{D}+1 / \kappa\right)-\chi\left(d_{D}-k_{0}-\delta_{D}\right)} \\
\cdot\left\|w_{1}(\tau, m)-w_{2}(\tau, m)\right\|_{(\nu, \beta, \mu, \chi, \alpha, \kappa, \epsilon)}
\end{gathered}
$$

for all $q_{1} \geq 1$ and $q_{2} \geq 1$ with $q_{1}+q_{2}=\delta_{D}$. Besides, from (137), we see that

$$
\begin{gathered}
\| \epsilon^{-\chi\left(d_{D}-k_{0}-\delta_{D}\right)} \frac{\widetilde{R}_{D}(i m)}{\widetilde{P}_{m}(\tau)} \tau^{\kappa} \times \int_{0}^{\tau^{\kappa}}\left(\tau^{\kappa}-s\right)^{d_{D, q_{1}, q_{2}} / \kappa+q_{2}-p-1} \\
\cdot s^{p}\left(w_{1}\left(s^{1 / \kappa}, m\right)-w_{2}\left(s^{1 / \kappa}, m\right)\right) \frac{d s}{s} \|_{(\nu, \beta, \mu, \chi, \alpha, \kappa, \epsilon)} \\
\leq \frac{C_{2}^{\prime}}{C_{\widetilde{P}}\left(r_{\widetilde{Q}, \widetilde{R}_{D}}\right)^{1 / \delta_{D} \kappa}}|\epsilon|^{(\chi+\alpha) \kappa\left(d_{D, q_{1}, q_{2}} / \kappa+q_{2}-\delta_{D}+1 / \kappa\right)-\chi\left(d_{D}-k_{0}-\delta_{D}\right)} \\
\cdot\left\|w_{1}(\tau, m)-w_{2}(\tau, m)\right\|_{(\nu, \beta, \mu, \chi, \alpha, \kappa, \epsilon)}
\end{gathered}
$$

provided that $q_{1} \geq 1, q_{2} \geq 2$, and $1 \leq p \leq q_{2}-1$ with $q_{1}+q_{2}=\delta_{D}$. Finally, having a glance at (138), we can select a constant $C_{2}^{\prime}>0$ (depending on $\nu, \kappa$, and $\delta_{D}$ ) such that

$$
\begin{aligned}
& \| \frac{\widetilde{R}_{D}(i m)}{\widetilde{P}_{m}(\tau)} \tau^{\kappa} \int_{0}^{\tau^{\kappa}}\left(\tau^{\kappa}-s\right)^{\delta_{D}-p-1} s^{p}\left(w_{1}\left(s^{1 / \kappa}, m\right)\right. \\
& \left.-w_{2}\left(s^{1 / \kappa}, m\right)\right) \frac{d s}{s} \|_{(\nu, \beta, \mu, \chi, \alpha, \kappa, \epsilon)} \\
& \leq \frac{C_{2}^{\prime}}{C_{\widetilde{P}}\left(r_{\widetilde{\mathrm{Q}}, \widetilde{R}_{D}}\right)^{1 / \delta_{D} \kappa}}|\epsilon|^{\chi+\alpha} \| w_{1}(\tau, m)-w_{2}(\tau,
\end{aligned}
$$

$m) \|_{(\nu, \beta, \mu, \chi, \alpha, \kappa, \epsilon)}$ for all $1 \leq p \leq \delta_{D}-1$. In the meanwhile, we select the size of radius $r_{\widetilde{Q}, \widetilde{R}_{D}}$ in such a manner that

$$
\begin{aligned}
& |\epsilon|^{\Delta_{D}+\alpha\left(\delta_{D}-d_{D}\right)+\beta}\left[\prod_{d=0}^{\delta_{D}^{-1}}|\gamma-d|\right. \\
& \cdot \frac{C_{2}}{C_{\widetilde{P}}\left(r_{\widetilde{Q}, \widetilde{R}_{D}}\right)^{1 / \delta_{D} \kappa} \Gamma\left(d_{D, \delta_{D}, 0} / \kappa\right)}|\epsilon|^{\alpha\left(d_{D}-k_{0}-\delta_{D}\right)} \\
& +\sum_{q_{1}+q_{2}=\delta_{D}, q_{1} \geq 1, q_{2} \geq 1} \frac{\delta_{D} !}{q_{1} ! q_{2} !} \prod_{d=0}^{q_{1}-1}|\gamma-d| \\
& \cdot\left(\frac{C_{2}^{\prime} \kappa^{q_{2}}}{C_{\widetilde{P}}\left(r_{\widetilde{Q}, \widetilde{R}_{D}}\right)^{1 / \delta_{D} \kappa} \Gamma\left(d_{D, q_{1}, q_{2}} / \kappa\right)}\right. \\
& \times|\epsilon|^{(\chi+\alpha) \kappa\left(d_{D, q_{1}, q_{2}} / \kappa+q_{2}-\delta_{D}+1 / \kappa\right)-\chi\left(d_{D}-k_{0}-\delta_{D}\right)} \\
& +\sum_{1 \leq p \leq q_{2}-1}\left|A_{q_{2}, p}\right| \\
& \cdot \frac{C_{2}^{\prime} \kappa^{p}}{C_{\widetilde{P}}\left(r_{\widetilde{Q}, \widetilde{R}_{D}}\right)^{1 / \delta_{D} \kappa} \Gamma\left(d_{D, q_{1}, q_{2}} / \kappa+q_{2}-p\right)} \\
& \left.\left.\times|\epsilon|^{(\chi+\alpha) \kappa\left(d_{D, q_{1}, q_{2}} / \kappa+q_{2}-\delta_{D}+1 / \kappa\right)-\chi\left(d_{D}-k_{0}-\delta_{D}\right)}\right)\right] \\
& +\sum_{1 \leq p \leq \delta_{D}-1}\left|A_{\delta_{D}, p}\right| \\
& \cdot \frac{C_{2}^{\prime} \kappa^{p}}{C_{\widetilde{P}}\left(r_{\widetilde{Q}, \widetilde{R}_{D}}\right)^{1 / \delta_{D} \kappa} \Gamma\left(\delta_{D}-p\right)}|\epsilon|^{\chi+\alpha} \leq \frac{1}{6} .
\end{aligned}
$$

The following inequality must then hold:

$$
\begin{aligned}
& \left\|\mathscr{H}_{\epsilon}^{3}\left(w_{1}(\tau, m)\right)-\mathscr{H}_{\epsilon}^{3}\left(w_{2}(\tau, m)\right)\right\|_{(\nu, \beta, \mu, \chi, \alpha, \kappa, \epsilon)} \\
& \leq \frac{1}{6}\left\|w_{1}(\tau, m)-w_{2}(\tau, m)\right\|_{(\nu, \beta, \mu, \chi, \alpha, \kappa, \epsilon)} .
\end{aligned}
$$

Gathering (144), (153), and (159) legitimates estimates (114).

At the very end of the proof, we now take for granted that all conditions (124), (133), (139), (143), (152), and (158) hold for the radii $r_{\widetilde{Q}, \widetilde{R}_{D}}$ and $\omega$. Then both (113) and (114) hold at the same time and Lemma 16 is shown.

We consider the closed ball $\bar{B}(0, \omega)$ just built above in Lemma 16 which is actually a complete metric space with respect to the metric induced by the Banach space norm $\|\cdot\|_{(\nu, \beta, \mu, \chi, \alpha, \kappa, \epsilon)}$. From the lemma above, we get that $\mathscr{H}_{\epsilon}$ is a contractive map from $\bar{B}(0, \omega)$ into itself. Due to the classical contractive mapping theorem, we deduce that the map $\mathscr{H}_{\epsilon}$ has a unique fixed point denoted by $\omega_{\kappa}^{d}(\tau, m, \epsilon)$ in the ball $\bar{B}(0, \omega)$, meaning that

$$
\mathscr{H}_{\epsilon}\left(\omega_{\kappa}^{d}(\tau, m, \epsilon)\right)=\omega_{\kappa}^{d}(\tau, m, \epsilon)
$$


for a unique $\omega_{\kappa}^{d}(\tau, m, \epsilon) \in F_{(\nu, \beta, \mu, \chi, \alpha, \kappa, \epsilon)}^{d}$ such that $\left\|\omega_{\kappa}^{d}(\tau, m, \epsilon)\right\|_{(\nu, \beta, \mu, \chi, \alpha, \kappa, \epsilon)} \leq \omega$, for all $\epsilon \in D\left(0, \epsilon_{0}\right) \backslash\{0\}$. Moreover, the function $\omega_{\kappa}^{d}(\tau, m, \epsilon)$ depends holomorphically on $\epsilon$ in $D\left(0, \epsilon_{0}\right) \backslash\{0\}$.

Now, if one sets apart the terms $-a_{0} \widetilde{Q}(i m) \omega_{\kappa}(\tau, m, \epsilon)$ in the left-hand side $L_{\tau, m, \epsilon}$ and $\widetilde{R}_{D}(i m)\left(\kappa \tau^{\kappa}\right)^{\delta_{D}} \omega_{\kappa}(\tau, m, \epsilon)$ in the right-hand side $R_{\tau, m, \epsilon}$ of (95), we observe by dividing with the polynomial $\widetilde{P}_{m}(\tau)$ given in (100) that (95) can be exactly rewritten as (160). Therefore, the unique fixed point $\omega_{\kappa}^{d}(\tau, m, \epsilon)$ of $\mathscr{H}_{\epsilon}$ in $\bar{B}(0, \omega)$ precisely solves problem (95) with vanishing initial data $\omega_{\kappa}(0, m, \epsilon) \equiv 0$. This yields the proposition.

\section{Singular Analytic Solutions on Sectors to the Main Problem}

We go back to the sequence of formal constructions performed in Section 4 under the new light shed in Section 5 on problem (95).

We first recall the definitions of a good covering and associated sets of sectors as introduced in [3].

Definition 17. Let $\varsigma \geq 2$ be an integer. For all $0 \leq p \leq \varsigma-1$, we consider open sectors $\mathscr{E}_{p}$ centered at 0 , with radius $\epsilon_{0}>0$ and opening $\pi /(\chi+\alpha) \kappa+\xi_{p}$ with $\xi_{p}>0$ small enough such that $\mathscr{E}_{p} \cap \mathscr{E}_{p+1} \neq \emptyset$, for all $0 \leq p \leq \varsigma-1$ (with the convention that $\left.\mathscr{E}_{\varsigma}=\mathscr{E}_{0}\right)$. Moreover, we assume that the intersection of any three different elements in $\left\{\mathscr{E}_{p}\right\}_{0 \leq p \leq \varsigma_{-1}}$ is empty and that $\cup_{p=0}^{\varsigma-1} \mathscr{E}_{p}=\mathscr{U} \backslash\{0\}$, where $\mathcal{U}$ is some neighborhood of 0 in $\mathbb{C}$. Such a set of sectors $\left\{\mathscr{E}_{p}\right\}_{0 \leq p \leq \varsigma-1}$ is called a good covering in $\mathbb{C}^{*}$.

Definition 18. Let $\left\{\mathscr{E}_{p}\right\}_{0 \leq p \leq \varsigma-1}$ be a good covering in $\mathbb{C}^{*}$. Let $\mathscr{T}$ be an open bounded sector centered at 0 with radius $r_{\mathscr{T}}$ and consider a family of open sectors

$$
\begin{aligned}
& S_{\mathfrak{D}_{p}, \theta, \epsilon_{0} r_{\mathscr{T}}} \\
& \quad=\left\{T \in \mathbb{C}^{*}|| T\left|<\epsilon_{0} r_{\mathscr{T}},\right| \mathfrak{D}_{p}-\arg (T) \mid<\frac{\theta}{2}\right\}
\end{aligned}
$$

with aperture $\theta>\pi / \kappa$ and where $\mathfrak{d}_{p} \in \mathbb{R}$, for all $0 \leq p \leq$ $\varsigma-1$, are directions which satisfy the following constraints: let $q_{l}(m)$ be the roots of the polynomials (100) defined by (101) and $S_{\mathfrak{b}_{p}}$ and $0 \leq p \leq \varsigma-1$ be unbounded sectors centered at 0 with directions $\mathfrak{d}_{p}$ and with small aperture. We assume the following.

(1) There exists a constant $M_{1}>0$ such that

$$
\left|\tau-q_{l}(m)\right| \geq M_{1}(1+|\tau|)
$$

for all $0 \leq l \leq \delta_{D} \kappa-1$; all $m \in \mathbb{R}$; all $\tau \in S_{\mathfrak{b}_{p}} \cup \bar{D}(0, \rho)$, for all $0 \leq p \leq \varsigma-1$.

(2) There exists a constant $M_{2}>0$ such that

$$
\left|\tau-q_{l_{0}}(m)\right| \geq M_{2}\left|q_{l_{0}}(m)\right|
$$

for some $l_{0} \in\left\{0, \ldots, \delta_{D} \kappa-1\right\}$; all $m \in \mathbb{R}$; all $\tau \in S_{\mathfrak{b}_{p}} \cup \bar{D}(0, \rho)$, for all $0 \leq p \leq \varsigma-1$.
(3) For all $0 \leq p \leq \varsigma-1$, all $t \in \mathscr{T}$; all $\epsilon \in \mathscr{E}_{p}$; we have

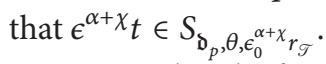

We say that the family $\left\{\left(S_{\mathfrak{S}_{p}, \theta, \epsilon_{0}} r_{\mathscr{T}}\right)_{0 \leq p \leq \varsigma-1}, \mathscr{T}\right\}$ is associated with the good covering $\left\{\mathscr{E}_{p}\right\}_{0 \leq p \leq \varsigma-1}$.

In the next main first outcome, we construct a family of actual holomorphic solutions to the principal equation (61) which may be meromorphic at $(\epsilon, t)=(0,0)$ and defined on the sectors $\mathscr{E}_{p}$ with respect to the complex parameter $\epsilon$. Furthermore, we can also control the difference between any two neighboring solutions on the intersections $\mathscr{E}_{p} \cap \mathscr{E}_{p+1}$ and state that it is exponentially flat of order at most $(\chi+\alpha) \kappa$ with respect to $\epsilon$.

Theorem 19. One considers the nonlinear singularly perturbed PDE (61) and takes for granted that all the assumptions (60), (62), (65), (66), (67), (77), (78), (79), (88), (99), (105), (106), and (107) hold for some rational numbers $\alpha>1, \beta \in \mathbb{Q}$ and integers $\gamma \in \mathbb{Z}, \kappa \geq 1$. Let $\left\{\mathscr{E}_{p}\right\}_{0 \leq p \leq \varsigma-1}$ a good covering in $\mathbb{C}^{*}$ be given, for which a family of open sectors $\left\{\left(S_{\mathfrak{b}_{p}, \theta, \epsilon_{0} r_{\mathscr{T}}}\right)_{0 \leq p \leq \varsigma-1}, \mathscr{T}\right\}$ associated with this good covering can be singled out.

Then, there exist a radius $r_{\widetilde{Q}, \widetilde{R}_{D}}>0$ large enough and $\epsilon_{0}>0$ small enough, for which a family $\left\{u^{\mathfrak{d}_{p}}(t, z, \epsilon)\right\}_{0 \leq p \leq c-1}$ of actual solutions of (61) can be built up. More exactly, the functions $u^{\mathfrak{d}_{p}}(t, z, \epsilon)$ solve the following singularly perturbed PDE:

$$
\begin{aligned}
& \widetilde{Q}\left(\partial_{z}\right)\left(\left(\sum_{l=0}^{q} a_{l} \epsilon^{m_{l}} t^{k_{l}}\right) u^{\delta_{p}}(t, z, \epsilon)\right. \\
& \left.+\left(\sum_{l=0}^{M} c_{l} \epsilon^{\mu_{l}} t^{h_{l}}\right)\left(u^{\delta_{p}}\right)^{2}(t, z, \epsilon)\right)=\sum_{j=0}^{p} \widetilde{b}_{j}(z) \epsilon^{n_{j}} t^{b_{j}} \\
& +F\left(\epsilon^{\alpha} t, \epsilon\right)+\sum_{l=1}^{D} \epsilon^{\Delta_{l}} t^{d_{l}} \partial_{t}^{\delta_{l}} \widetilde{R}_{l}\left(\partial_{z}\right) u(t, z, \epsilon)
\end{aligned}
$$

with an additional part of forcing term $F\left(\epsilon^{\alpha} t, \epsilon\right)$ where $F(T, \epsilon)$ is given by expression (183) and defines a holomorphic bounded function provided that the additional constraints (184) are fulfilled. Each function $u^{\mathfrak{b}_{p}}(t, z, \epsilon)$ can be decomposed as

$$
\begin{aligned}
& u^{\mathfrak{d}_{p}}(t, z, \epsilon)=\epsilon^{\beta}\left(-\frac{a_{0}}{c_{0}}\left(\epsilon^{\alpha} t\right)^{k_{0}-h_{0}}\right. \\
& \left.-\frac{a_{0}}{c_{0}}\left(\epsilon^{\alpha} t\right)^{k_{0}-h_{0}} J\left(\epsilon^{\alpha} t\right)+\left(\epsilon^{\alpha} t\right)^{\gamma} v^{\delta_{p}}(t, z, \epsilon)\right),
\end{aligned}
$$

where $J(T)$ is holomorphic on some disc $D\left(0, d_{J}\right), d_{J}>0$, and $v^{\delta_{p}}(t, z, \epsilon)$ defines a bounded holomorphic function on $\mathscr{T} \times H_{\beta^{\prime}} \times \mathscr{E}_{p}$ for any given $0<\beta^{\prime}<\beta$, with $v^{\mathfrak{b}_{p}}(0, z, \epsilon) \equiv 0$ on $H_{\beta^{\prime}} \times \mathscr{E}_{p}$. Furthermore, there exist constants $K_{p}, M_{p}>0$ and $\sigma>0$ (independent of $\epsilon$ ) such that

$$
\begin{aligned}
& \sup _{t \in \mathscr{T} \cap D(0, \sigma), z \in H_{\beta^{\prime}}}\left|v^{\mathfrak{D}_{p+1}}(t, z, \epsilon)-v^{\mathfrak{\delta}_{p}}(t, z, \epsilon)\right| \\
& \leq K_{p} \exp \left(-\frac{M_{p}}{|\epsilon|^{(\chi+\alpha) \kappa}}\right)
\end{aligned}
$$


for all $\epsilon \in \mathscr{E}_{p+1} \cap \mathscr{E}_{p}$, for all $0 \leq p \leq \varsigma-1$ (where by convention $\left.v^{\mathfrak{\delta}_{\varsigma}}=v^{\mathfrak{\delta}_{0}}\right)$.

Proof. We plan to construct actual solutions of the main equation (61) by performing backwards the sequence of constructions described in Section 4 starting from problem (95) solved in Section 5.

Let $\left\{\mathscr{E}_{p}\right\}_{0 \leq p \leq \varphi^{-1}}$ be a good covering in $\mathbb{C}^{*}$ and let $\left\{\left(S_{\mathfrak{D}_{p}, \theta, \epsilon_{0} r_{\mathscr{T}}}\right)_{0 \leq p \leq \varsigma-1}, \mathscr{T}\right\}$ be a family of sectors associated with this good covering. From Proposition 15, we see that, for each direction $\mathfrak{b}_{p}$, one can get a solution $\omega_{\kappa}^{\mathfrak{o}_{p}}(\tau, m, \epsilon)$ of the convolution equation (95) that belongs to the space $F_{(\nu, \beta, \mu, \chi, \alpha, \kappa, \epsilon)}^{\mathcal{\delta}_{p}}$ and thus satisfies the following bounds:

$$
\begin{aligned}
\left|\omega_{\kappa}^{\mathfrak{o}_{p}}(\tau, m, \epsilon)\right| \leq & \oplus(1+|m|)^{-\mu} e^{-\beta|m|} \frac{\left|\tau / \epsilon^{\chi+\alpha}\right|}{1+\left|\tau / \epsilon^{\chi+\alpha}\right|^{2 \kappa}} \\
& \cdot \exp \left(\nu\left|\frac{\tau}{\epsilon^{\chi+\alpha}}\right|^{\kappa}\right)
\end{aligned}
$$

for all $\tau \in \bar{D}(0, \rho) \cup S_{\mathfrak{D}_{p}}$, all $m \in \mathbb{R}$, and all $\epsilon \in D\left(0, \epsilon_{0}\right) \backslash\{0\}$, for some well chosen $\omega>0$. Besides, these functions $\omega_{\kappa}^{\mathfrak{d}_{p}}(\tau, m, \epsilon)$ are analytic continuations with respect to $\tau$ of a common convergent series

$$
\omega_{\kappa}(\tau, m, \epsilon)=\sum_{n \geq 1} \frac{\omega_{n}(m, \epsilon)}{\Gamma(n / \kappa)} \tau^{n}
$$

with coefficients in the Banach space $E_{(\beta, \mu)}$ solution of (95) for all $\tau \in D(0, \rho)$. In particular, we see that the formal power series

$$
\Omega_{\kappa}(\mathbb{T}, m, \epsilon)=\sum_{n \geq 1} \omega_{n}(m, \epsilon) \mathbb{T}^{n}
$$

is $m_{\kappa}$-summable in direction $\mathfrak{d}_{p}$ as a series with coefficients in the Banach space $E_{(\beta, \mu)}$ for all $\epsilon \in D\left(0, \epsilon_{0}\right) \backslash\{0\}$ in the sense of Definition 7. We denote by

$$
\Omega_{\kappa}^{\mathfrak{d}_{p}}(\mathbb{T}, m, \epsilon)=\kappa \int_{L_{\gamma}} \omega_{\kappa}^{\mathfrak{d}_{p}}(u, m, \epsilon) \exp \left(-\left(\frac{u}{\mathbb{T}}\right)^{\kappa}\right) \frac{d u}{u}
$$

its $m_{\mathcal{\kappa}}$-sum in direction $\mathfrak{d}_{p}$, where $L_{\gamma}=\mathbb{R}_{+} e^{i \gamma} \subset S_{\mathfrak{b}_{p}}$, which defines an $E_{(\beta, \mu)}$-valued analytic function with respect to $\mathbb{T}$ on a sector

$$
\begin{aligned}
& S_{\mathfrak{d}_{p}, \theta, h^{\prime}|\epsilon|^{\chi+\alpha}} \\
& \quad=\left\{\mathbb{T} \in \mathbb{C}^{*}:|\mathbb{T}|<h^{\prime}|\epsilon|^{\chi+\alpha},\left|\mathfrak{b}_{p}-\arg (\mathbb{T})\right|<\frac{\theta}{2}\right\}
\end{aligned}
$$

for $\pi / \kappa<\theta<\pi / \kappa+\operatorname{Ap}\left(S_{\mathfrak{b}_{p}}\right)$ (where $\operatorname{Ap}\left(S_{\mathfrak{b}_{p}}\right)$ denotes the aperture of the sector $S_{\mathfrak{D}_{p}}$ ) and some $h^{\prime}>0$ (independent of $\epsilon)$, for all $\epsilon \in D\left(0, \epsilon_{0}\right) \backslash\{0\}$.

Bearing in mind the identities of Proposition 8 and using the properties for the $m_{\kappa}$-sum with respect to derivatives and products (within the Banach algebra $\mathbb{E}=E_{(\beta, \mu)}$ equipped with the convolution product $\star$ as described in Proposition 2), we check that the functions $\Omega_{\kappa}^{\mathfrak{d}_{p}}(\mathbb{T}, m, \epsilon)$ must solve the following problem:

$$
\mathscr{L}_{\mathbb{T}, m, \epsilon}\left(\Omega_{\kappa}^{\mathfrak{d}_{p}}(\mathbb{T}, m, \epsilon)\right)=\mathscr{R}_{\mathbb{T}, m, \epsilon}\left(\Omega_{\kappa}^{\mathfrak{d}_{p}}(\mathbb{T}, m, \epsilon)\right),
$$

where

$$
\begin{aligned}
& \mathscr{L}_{\mathbb{T}, m, \epsilon}\left(\Omega_{\kappa}^{\mathfrak{d}_{p}}(\mathbb{T}, m, \epsilon)\right)=\widetilde{Q}(i m) \Omega_{\kappa}^{\mathfrak{d}_{p}}(\mathbb{T}, m, \epsilon)\left(-a_{0}\right. \\
& +\sum_{l=1}^{s} a_{l} \epsilon^{-\chi\left(k_{l}-k_{0}\right)} \mathbb{T}^{k_{l}-k_{0}} \\
& +\sum_{l=s+1}^{q} a_{l} \epsilon^{m_{l}+\beta-\alpha k_{l}-\chi\left(k_{l}-k_{0}\right)} \mathbb{T}^{k_{l}-k_{0}}-2\left(\frac{a_{0}}{c_{0}}\right) \\
& \cdot\left(\sum_{l=1}^{s^{\prime}} c_{l} \epsilon^{-\chi\left(h_{l}-h_{0}\right)} \mathbb{T}^{h_{l}-h_{0}}\right. \\
& \left.+\sum_{l=s^{\prime}+1}^{M} c_{l} \epsilon^{\mu_{l}+2 \beta-\alpha h_{l}-\chi\left(h_{l}-h_{0}\right)} \mathbb{T}^{h_{l}-h_{0}}\right)-2\left(\frac{a_{0}}{c_{0}}\right) \\
& \cdot J\left(\epsilon^{-\chi} \mathbb{T}\right)\left(\sum_{l=0}^{s^{\prime}} \mathcal{c}_{l} \epsilon^{-\chi\left(h_{l}-h_{0}\right)} \mathbb{T}^{h_{l}-h_{0}}\right. \\
& \left.\left.+\sum_{l=s^{\prime}+1}^{M} c_{l} \epsilon^{\mu_{l}+2 \beta-\alpha h_{l}-\chi\left(h_{l}-h_{0}\right)} \mathbb{T}^{h_{l}-h_{0}}\right)\right)+\widetilde{Q}(i m) \\
& \cdot\left(\frac{1}{(2 \pi)^{1 / 2}} \int_{-\infty}^{+\infty} \Omega_{\kappa}^{\mathfrak{d}_{p}}\left(\mathbb{T}, m-m_{1}, \epsilon\right)\right. \\
& \left.\cdot \Omega_{\kappa}^{\mathfrak{d}_{p}}\left(\mathbb{T}, m_{1}, \epsilon\right) d m_{1}\right) \epsilon^{-\chi\left(-k_{0}+\gamma\right)} \mathbb{T}^{-k_{0}+\gamma} \\
& \times\left(\sum_{l=0}^{s^{\prime}} c_{l} \epsilon^{-\chi h_{l}} \mathbb{T}^{h_{l}}+\sum_{l=s^{\prime}+1}^{M} c_{l} \epsilon^{\mu_{l}+2 \beta-\alpha h_{l}-\chi h_{l}} \mathbb{T}^{h_{l}}\right), \\
& \mathscr{R}_{\mathbb{T}, m, \epsilon}\left(\Omega_{\kappa}^{\mathfrak{d}_{p}}(\mathbb{T}, m, \epsilon)\right)=\sum_{j=0}^{p} \widetilde{B}_{j}(m) \\
& \cdot \epsilon^{n_{j}-\alpha b_{j}-\chi\left(b_{j}-k_{0}-\gamma\right)} \mathbb{T}^{b_{j}-k_{0}-\gamma}+\sum_{l=1}^{D-1} \epsilon^{\Delta_{l}+\alpha\left(\delta_{l}-d_{l}\right)+\beta} \\
& \times \sum_{q_{1}+q_{2}=\delta_{l}} \frac{\delta_{l} !}{q_{1} ! q_{2} !} \prod_{d=0}^{q_{1}-1}(\gamma-d) \epsilon^{-\chi\left(d_{l}-k_{0}-q_{1}-q_{2}\right)} \widetilde{R}_{l}(i m) \\
& \times \mathbb{T}^{d_{l, q_{1}, q_{2}}}\left\{\left(\mathbb{T}^{\kappa+1} \partial_{\mathbb{T}}\right)^{q_{2}}+\sum_{1 \leq p \leq q_{2}-1} A_{q_{2}, p} \mathbb{T}^{\kappa\left(q_{2}-p\right)}\right. \\
& \left.\cdot\left(\mathbb{T}^{\kappa+1} \partial_{\mathbb{T}}\right)^{p}\right\} \Omega_{\kappa}^{\mathfrak{d}_{p}}(\mathbb{T}, m, \epsilon)+\epsilon^{\Delta_{D}+\alpha\left(\delta_{D}-d_{D}\right)+\beta}
\end{aligned}
$$




$$
\begin{aligned}
& \times \sum_{q_{1}+q_{2}=\delta_{D}, q_{1} \geq 1} \frac{\delta_{D} !}{q_{1} ! q_{2} !} \prod_{d=0}^{q_{1}-1}(\gamma-d) \\
& \cdot \epsilon^{-\chi\left(d_{D}-k_{0}-q_{1}-q_{2}\right)} \widetilde{R}_{D}(i m) \times \mathbb{T}^{d_{D, q_{1}, q_{2}}}\left\{\left(\mathbb{T}^{\kappa+1} \partial_{\mathbb{T}}\right)^{q_{2}}\right. \\
& \left.+\sum_{1 \leq p \leq q_{2}-1} A_{q_{2}, p} \mathbb{T}^{\kappa\left(q_{2}-p\right)}\left(\mathbb{T}^{\kappa+1} \partial_{\mathbb{T}}\right)^{p}\right\} \Omega_{\kappa}^{\mathfrak{d}_{p}}(\mathbb{T}, m, \epsilon) \\
& +\widetilde{R}_{D}(i m)\left\{\left(\mathbb{T}^{\kappa+1} \partial_{\mathbb{T}}\right)^{\delta_{D}}+\sum_{1 \leq p \leq \delta_{D}-1} A_{\delta_{D}, p} \mathbb{T}^{\kappa\left(\delta_{D}-p\right)}\right. \\
& \left.\cdot\left(\mathbb{T}^{\kappa+1} \partial_{\mathbb{T}}\right)^{p}\right\} \Omega_{\kappa}^{\mathfrak{d}_{p}}(\mathbb{T}, m, \epsilon) .
\end{aligned}
$$

$$
\begin{aligned}
& \cdot \epsilon^{n_{j}-\alpha b_{j}-\chi\left(b_{j}-k_{0}-\gamma\right)} \mathbb{T}^{b_{j}-k_{0}-\gamma}+\sum_{l=1}^{D-1} \epsilon^{\Delta_{l}+\alpha\left(\delta_{l}-d_{l}\right)+\beta} \\
& \times \sum_{q_{1}+q_{2}=\delta_{l}} \frac{\delta_{l} !}{q_{1} ! q_{2} !} \prod_{d=0}^{q_{1}-1}(\gamma-d) \epsilon^{-\chi\left(d_{l}-k_{0}-q_{1}\right)} \mathbb{T}^{d_{l}-k_{0}-q_{1}} \\
& \cdot \widetilde{R}_{l}\left(\partial_{z}\right) \epsilon^{\chi q_{2}} \partial_{\mathbb{T}}^{q_{2}} \mathbb{V}^{\delta_{p}}(\mathbb{T}, z, \epsilon) \\
& +\epsilon^{\Delta_{D}+\alpha\left(\delta_{D}-d_{D}\right)+\beta} \sum_{q_{1}+q_{2}=\delta_{D}, q_{1} \geq 1} \frac{\delta_{D} !}{q_{1} ! q_{2} !} \prod_{d=0}^{q_{1}-1}(\gamma-d) \\
& \cdot \epsilon^{-\chi\left(d_{D}-k_{0}-q_{1}\right)} \mathbb{T}^{d_{D}-k_{0}-q_{1}} \times \widetilde{R}_{D}\left(\partial_{z}\right) \epsilon^{\chi q_{2}} \partial_{\mathbb{T}}^{q_{2}} \mathbb{V}^{\delta_{p}}(\mathbb{T}, z, \\
& \epsilon)+\mathbb{T}^{d_{D}-k_{0}} \widetilde{R}_{D}\left(\partial_{z}\right) \partial_{\mathbb{T}}^{\delta_{D}} \mathbb{V}^{\delta_{p}}(\mathbb{T}, z, \epsilon) .
\end{aligned}
$$

We examine now the function

$$
\mathbb{V}^{\mathfrak{d}_{p}}(\mathbb{T}, z, \epsilon)=\mathscr{F}^{-1}\left(m \longmapsto \Omega_{\kappa}^{\mathfrak{d}_{p}}(\mathbb{T}, m, \epsilon)\right)(z)
$$

which defines a bounded holomorphic function with respect to $\mathbb{T}$ on $S_{\mathfrak{b}_{p}, \theta, h^{\prime}|\epsilon|^{\mid+\alpha}}$, with respect to $z$ on $H_{\beta^{\prime}}$ for any $0<$ $\beta^{\prime}<\beta$, and for all $\epsilon$ on $D\left(0, \epsilon_{0}\right) \backslash\{0\}$. Using the properties of the Fourier inverse transform described in Proposition 9 and watching out the expansions (91), we extract from equality (172) the next equation satisfied by $\mathbb{V}^{\mathfrak{d}_{p}}(\mathbb{T}, z, \epsilon)$; namely,

$$
\begin{aligned}
& \widetilde{Q}\left(\partial_{z}\right) \mathbb{V}^{\delta_{p}}(\mathbb{T}, z, \epsilon)\left(-a_{0}+\sum_{l=1}^{s} a_{l} \epsilon^{-\chi\left(k_{l}-k_{0}\right)} \mathbb{T}^{k_{l}-k_{0}}\right. \\
& +\sum_{l=s+1}^{q} a_{l} \epsilon^{m_{l}+\beta-\alpha k_{l}-\chi\left(k_{l}-k_{0}\right)} \mathbb{T}^{k_{l}-k_{0}}-2\left(\frac{a_{0}}{c_{0}}\right) \\
& \cdot\left(\sum_{l=1}^{s^{\prime}} \mathcal{c}_{l} \epsilon^{-\chi\left(h_{l}-h_{0}\right)} \mathbb{T}^{h_{l}-h_{0}}\right. \\
& \left.+\sum_{l=s^{\prime}+1}^{M} c_{l} \epsilon^{\mu_{l}+2 \beta-\alpha h_{l}-\chi\left(h_{l}-h_{0}\right)} \mathbb{T}^{h_{l}-h_{0}}\right)-2\left(\frac{a_{0}}{c_{0}}\right) \\
& \cdot J\left(\epsilon^{-\chi} \mathbb{T}\right)\left(\sum_{l=0}^{s^{\prime}} c_{l} \epsilon^{-\chi\left(h_{l}-h_{0}\right)} \mathbb{T}^{h_{l}-h_{0}}\right. \\
& \left.\left.+\sum_{l=s^{\prime}+1}^{M} c_{l} \epsilon^{\mu_{l}+2 \beta-\alpha h_{l}-\chi\left(h_{l}-h_{0}\right)} \mathbb{T}^{h_{l}-h_{0}}\right)\right)+\widetilde{Q}\left(\partial_{z}\right) \\
& \quad \cdot\left(\mathbb{V}^{\delta_{p}}\right)^{2}(\mathbb{T}, z, \epsilon) \epsilon^{-\chi\left(-k_{0}+\gamma\right)} \mathbb{T}^{-k_{0}+\gamma}\left(\sum_{l=0}^{s^{\prime}} \mathcal{c}_{\epsilon} \epsilon^{-\chi h_{l}} \mathbb{T}^{h_{l}}\right. \\
& \left.+\sum_{l=s^{\prime}+1}^{M} c_{l} \epsilon^{\mu_{l}+2 \beta-\alpha h_{l}-\chi h_{l}} \mathbb{T}^{h_{l}}\right)=\sum_{j=0}^{p} \widetilde{b}_{j}(z)
\end{aligned}
$$

We now set $\mathbb{T}=\epsilon^{\chi} T$ and we focus on the function

$$
V^{\mathfrak{d}_{p}}(T, z, \epsilon)=\mathbb{V}^{\mathfrak{d}_{p}}\left(\epsilon^{\chi} T, z, \epsilon\right)
$$

which defines a bounded holomorphic function with respect to $T$ such that $T \in \epsilon^{-\chi} S_{\mathfrak{D}_{p}, \theta, h^{\prime}|\epsilon| \chi^{+\alpha}}$ and with respect to $z$ on $H_{\beta^{\prime}}$ for any $0<\beta^{\prime}<\beta$, for all $\epsilon \in D\left(0, \epsilon_{0}\right) \backslash\{0\}$. Having a quick look at (176), we observe that $V^{\mathfrak{d}_{p}}(T, z, \epsilon)$ solves a related equation which after multiplication by $T^{k_{0}+\gamma}$ yields

$$
\begin{aligned}
\widetilde{Q} & \left(\partial_{z}\right) V^{\mathfrak{d}_{p}}(T, z, \epsilon)\left(-a_{0} T^{k_{0}+\gamma}\right. \\
+ & \left(\sum_{l=1}^{s} a_{l} T^{k_{l}}+\sum_{l=s+1}^{q} a_{l} \epsilon^{m_{l}+\beta-\alpha k_{l}} T^{k_{l}}\right) T^{\gamma}-2\left(\frac{a_{0}}{c_{0}}\right) \\
& \cdot T^{k_{0}-h_{0}+\gamma}\left(\sum_{l=1}^{s^{\prime}} c_{l} T^{h_{l}}+\sum_{l=s^{\prime}+1}^{M} c_{l} \epsilon^{\mu_{l}+2 \beta-\alpha h_{l}} T^{h_{l}}\right) \\
& -2\left(\frac{a_{0}}{c_{0}}\right) T^{k_{0}-h_{0}+\gamma} J(T) \\
& \left.\cdot\left(\sum_{l=0}^{s^{\prime}} c_{l} T^{h_{l}}+\sum_{l=s^{\prime}+1}^{M} c_{l} \epsilon^{\mu_{l}+2 \beta-\alpha h_{l}} T^{h_{l}}\right)\right)+\widetilde{Q}\left(\partial_{z}\right) \\
& \cdot\left(V^{\delta_{p}}\right)^{2}(T, z, \epsilon) T^{2 \gamma}\left(\sum_{l=0}^{s^{\prime}} c_{l} T^{h_{l}}\right. \\
& \left.+\sum_{l=s^{\prime}+1}^{M} c_{l} \epsilon^{\mu_{l}+2 \beta-\alpha h_{l}} T^{h_{l}}\right)=\sum_{j=0}^{p} \widetilde{b}_{j}(z) \epsilon^{n_{j}-\alpha b_{j}} T^{b_{j}} \\
& +\sum_{l=1}^{D} \epsilon^{\Delta_{l}+\alpha\left(\delta_{l}-d_{l}\right)+\beta}\left(\sum_{q_{1}+q_{2}=\delta_{l}} \frac{\delta_{l} !}{q_{1} ! q_{2} !}\right. \\
& \left.\cdot \prod_{d=0}^{q_{1}-1}(\gamma-d) T^{d_{l}+\gamma-q_{1}} \widetilde{R}_{l}\left(\partial_{z}\right) \partial_{T}^{q_{2}} V^{\mathfrak{\delta}_{p}}(T, z, \epsilon)\right) .
\end{aligned}
$$


In the next step, we introduce the function

$$
\begin{aligned}
U^{\mathfrak{\jmath}_{p}}(T, z, \epsilon)= & -\frac{a_{0}}{c_{0}} T^{k_{0}-h_{0}}-\frac{a_{0}}{c_{0}} T^{k_{0}-h_{0}} J(T) \\
& +T^{\gamma} V^{\mathfrak{d}_{p}}(T, z, \epsilon)
\end{aligned}
$$

which defines a holomorphic function with respect to $T$ such that $T \in \epsilon^{-\chi} S_{\mathfrak{S}_{p}, \theta, h^{\prime}|\epsilon|^{\chi+\alpha}}$ and with respect to $z$ on $H_{\beta^{\prime}}$ for any $0<\beta^{\prime}<\beta$, for all $\epsilon \in D\left(0, \epsilon_{0}\right) \backslash\{0\}$. Notice that this function may be meromorphic at $T=0$, provided that $h_{0}>k_{0}$. Taking (178) into consideration, we see that the function $U^{\mathfrak{b}_{p}}(T, z, \epsilon)$ solves the next PDE with forcing term

$$
\begin{aligned}
\widetilde{Q} & \left(\partial_{z}\right)\left(\left(\sum_{l=0}^{q} a_{l} \epsilon^{m_{l}+\beta-\alpha k_{l}} T^{k_{l}}\right) U^{\mathfrak{\delta}_{p}}(T, z, \epsilon)\right. \\
+ & \left.\left(\sum_{l=0}^{M} c_{l} \epsilon^{\mu_{l}+2 \beta-\alpha h_{l}} T^{h_{l}}\right)\left(U^{\mathfrak{\delta}_{p}}\right)^{2}(T, z, \epsilon)\right) \\
= & \sum_{j=0}^{p} \widetilde{b}_{j}(z) \epsilon^{n_{j}-\alpha b_{j}} T^{b_{j}}+F(T, \epsilon) \\
+ & \sum_{l=1}^{D} \epsilon^{\Delta_{l}+\alpha\left(\delta_{l}-d_{l}\right)+\beta} T^{d_{l}} \widetilde{R}_{l}\left(\partial_{z}\right) \partial_{T}^{\delta_{l}} U^{\mathfrak{\delta}_{p}}(T, z, \epsilon)
\end{aligned}
$$

which is exactly (72) announced in Remark 12 of Section 4.1, where $F(T, \epsilon)$ is a contribution to the forcing term equal to

$$
\begin{aligned}
F(T, \epsilon)=-\widetilde{Q}(0)\left(\frac{a_{0}}{c_{0}} T^{k_{0}-h_{0}}+\frac{a_{0}}{c_{0}} T^{k_{0}-h_{0}} J(T)\right) \\
\times\left(-a_{0} T^{k_{0}}+\left(\sum_{l=1}^{s} a_{l} T^{k_{l}}+\sum_{l=s+1}^{q} a_{l} \epsilon^{m_{l}+\beta-\alpha k_{l}} T^{k_{l}}\right)\right. \\
-2\left(\frac{a_{0}}{c_{0}}\right) T^{k_{0}-h_{0}}\left(\sum_{l=1}^{s^{\prime}} c_{l} T^{h_{l}}+\sum_{l=s^{\prime}+1}^{M} c_{l} \epsilon^{\mu_{l}+2 \beta-\alpha h_{l}} T^{h_{l}}\right) \\
-2\left(\frac{a_{0}}{c_{0}}\right) T^{k_{0}-h_{0}} J(T) \\
\left.\cdot\left(\sum_{l=0}^{s^{\prime}} c_{l} T^{h_{l}}+\sum_{l=s^{\prime}+1}^{M} c_{l} \epsilon^{\mu_{l}+2 \beta-\alpha h_{l}} T^{h_{l}}\right)\right)-\widetilde{Q}(0)\left(\frac{a_{0}}{c_{0}}\right. \\
\left.\cdot T^{k_{0}-h_{0}}+\frac{a_{0}}{c_{0}} T^{k_{0}-h_{0}} J(T)\right)^{2}\left(\sum_{l=0}^{s^{\prime}} c_{l} T^{h_{l}}\right. \\
+\sum_{T}^{\delta_{l}}\left(\frac{a_{0}}{c_{0}} T^{k_{0}-h_{0}}+\frac{a_{0}}{c_{0}} T^{c_{0}-h_{0}} J(T)\right) . \\
\left.+\epsilon^{\mu_{l}+2 \beta-\alpha h_{l}} T^{h_{l}}\right)+\sum_{l=1}^{D} \epsilon^{\Delta_{l}+\alpha\left(\delta_{l}-d_{l}\right)+\beta} T^{d_{l}} \widetilde{R}_{l}(0)
\end{aligned}
$$

Using the fact that $U_{0}(T)$ solves the second-order algebraic equation (70) and noticing the following identity:

$$
\begin{array}{r}
-a_{0} T^{k_{0}}+\sum_{l=1}^{s} a_{l} T^{k_{l}}-2\left(\frac{a_{0}}{c_{0}}\right) T^{k_{0}-h_{0}}\left(\sum_{l=1}^{s^{\prime}} c_{l} T^{h_{l}}\right) \\
=\left(\sum_{l=0}^{s} a_{l} T^{k_{l}}\right)-2\left(\frac{a_{0}}{c_{0}}\right) T^{k_{0}-h_{0}}\left(\sum_{l=0}^{s^{\prime}} c_{l} T^{h_{l}}\right),
\end{array}
$$

we can abridge the latter expression of $F(T, \epsilon)$ as

$$
\begin{aligned}
& F(T, \epsilon)=\widetilde{Q}(0) U_{0}(T)\left(\left(\sum_{l=s+1}^{q} a_{l} \epsilon^{m_{l}+\beta-\alpha k_{l}} T^{k_{l}}\right)\right. \\
& -2\left(\frac{a_{0}}{c_{0}}\right) T^{k_{0}-h_{0}}\left(\sum_{l=s^{\prime}+1}^{M} c_{l} \epsilon^{\mu_{l}+2 \beta-\alpha h_{l}} T^{h_{l}}\right) \\
& \left.-2\left(\frac{a_{0}}{c_{0}}\right) T^{k_{0}-h_{0}} J(T)\left(\sum_{l=s^{\prime}+1}^{M} c_{l} \epsilon^{\mu_{l}+2 \beta-\alpha h_{l}} T^{h_{l}}\right)\right) \\
& -\widetilde{Q}(0) U_{0}^{2}(T)\left(\sum_{l=s^{\prime}+1}^{M} c_{l} \epsilon^{\mu_{l}+2 \beta-\alpha h_{l}} T^{h_{l}}\right) \\
& +\sum_{l=1}^{D} \epsilon^{\Delta_{l}+\alpha\left(\delta_{l}-d_{l}\right)+\beta} T^{d_{l}} \widetilde{R}_{l}(0) \partial_{T}^{\delta_{l}}\left(\frac{a_{0}}{c_{0}} T^{k_{0}-h_{0}}\right. \\
& \left.+\frac{a_{0}}{c_{0}} T^{k_{0}-h_{0}} J(T)\right) .
\end{aligned}
$$

Observe that $F(T, \epsilon)$ is bounded holomorphic with respect to $\epsilon$ and is analytic in $T$ near 0 provided that the following additional conditions hold:

$$
\begin{aligned}
k_{l}+k_{0}-h_{0} & \geq 0, \\
h_{p}+2\left(k_{0}-h_{0}\right) & \geq 0, \\
d_{m}+k_{0}-h_{0}-\delta_{m} & \geq 0
\end{aligned}
$$

for all $s+1 \leq l \leq q, s^{\prime}+1 \leq p \leq M$, and $1 \leq m \leq D$.

Finally, we put

$$
\begin{aligned}
u^{\mathfrak{d}_{p}}(t, z, \epsilon) & =\epsilon^{\beta} U^{\mathfrak{d}_{p}}\left(\epsilon^{\alpha} t, z, \epsilon\right) \\
& =\epsilon^{\beta}\left(U_{0}\left(\epsilon^{\alpha} t\right)+\left(\epsilon^{\alpha} t\right)^{\gamma} \mathbb{V}^{\mathfrak{d}_{p}}\left(\epsilon^{\chi+\alpha} t, z, \epsilon\right)\right)
\end{aligned}
$$

which defines a holomorphic function with respect to $t$ on $\mathscr{T}$, with respect to $z \in H_{\beta^{\prime}}$ for any $0<\beta^{\prime}<\beta$, and with respect to $\epsilon \in \mathscr{E}_{p}$, where $\mathscr{T}$ and $\mathscr{E}_{p}$ are sectors described in Definition 18. As a result, $u^{\mathfrak{d}_{p}}(t, z, \epsilon)$ admits the decomposition (165) with $v^{\mathfrak{d}_{p}}(t, z, \epsilon)=\mathbb{V}^{\mathfrak{d}_{p}}\left(\epsilon^{\chi+\alpha} t, z, \epsilon\right)$ which determines a bounded holomorphic function on $\mathscr{T} \times H_{\beta^{\prime}} \times \mathscr{E}_{p}$ for any given $0<\beta^{\prime}<\beta$ with the property $v^{\delta_{p}}(0, z, \epsilon) \equiv 0$ for all $(z, \epsilon) \in H_{\beta^{\prime}} \times \mathscr{E}_{p}$. Again, the function $u^{\mathfrak{d}_{p}}(t, z, \epsilon)$ may be meromorphic in both $t$ and $\epsilon$ in the vicinity of the origin. 
From $(180)$ and (183) we deduce that $u^{\mathfrak{d}_{p}}(t, z, \epsilon)$ solves the next main problem

$$
\begin{aligned}
& \widetilde{Q}\left(\partial_{z}\right)\left(\left(\sum_{l=0}^{q} a_{l} \epsilon^{m_{l}} t^{k_{l}}\right) u^{\mathfrak{d}_{p}}(t, z, \epsilon)\right. \\
& \left.+\left(\sum_{l=0}^{M} \mathcal{c}_{l} \epsilon^{\mu_{l}} t^{h_{l}}\right)\left(u^{\mathfrak{d}_{p}}\right)^{2}(t, z, \epsilon)\right)=\sum_{j=0}^{p} \widetilde{b}_{j}(z) \epsilon^{n_{j}} t^{b_{j}} \\
& +F\left(\epsilon^{\alpha} t, \epsilon\right)+\sum_{l=1}^{D} \epsilon^{\Delta_{l}} t^{d_{l}} \partial_{t}^{\delta_{l}} \widetilde{R}_{l}\left(\partial_{z}\right) u^{\mathfrak{\delta}_{p}}(t, z, \epsilon)
\end{aligned}
$$

with additional forcing term $F\left(\epsilon^{\alpha} t, \epsilon\right)$. As a spin-off, by applying the operator $\partial_{z}^{v}$ on the left- and right-hand side of this last equation, we see that $u^{\delta_{p}}(t, z, \epsilon)$ is also an actual solution of problem (61) disclosed at the beginning of Section 4.

In the last part of the proof, we proceed to justify estimates (166). The steps of the verification are similar to the arguments displayed in Theorem 1 of [3], but we choose to present them for the sake of completeness. Let $p \in\{0, \ldots, \varsigma-$ $1\}$. By the sequence of constructions performed above, we see that the function $v^{\delta_{p}}(t, z, \epsilon)$ can be written as a $m_{\kappa}$-Laplace and Fourier transform

$$
\begin{gathered}
v^{\mathfrak{d}_{p}}(t, z, \epsilon)=\frac{\kappa}{(2 \pi)^{1 / 2}} \int_{-\infty}^{+\infty} \int_{L_{\gamma_{p}}} \omega_{\mathcal{\kappa}}^{\mathfrak{d}_{p}}(u, m, \epsilon) \\
\cdot \exp \left(-\left(\frac{u}{\epsilon^{\chi+\alpha} t}\right)^{\kappa}\right) e^{i z m} \frac{d u}{u} d m,
\end{gathered}
$$

where $L_{\gamma_{p}}=\mathbb{R}_{+} e^{i \gamma_{p}} \subset S_{\mathfrak{D}_{p}}$. Using the fact that the function $u \mapsto \omega_{\kappa}(u, m, \epsilon) \exp \left(-\left(u / \epsilon^{\chi+\alpha} t\right)^{\kappa}\right) / u$ is holomorphic on $D(0, \rho)$ for all $(m, \epsilon) \in \mathbb{R} \times\left(D\left(0, \epsilon_{0}\right) \backslash\{0\}\right)$, its integral along the union of a segment starting from 0 to $(\rho / 2) e^{i \gamma_{p+1}}$, an arc of circle with radius $\rho / 2$ which connects $(\rho / 2) e^{i \gamma_{p+1}}$ and $(\rho / 2) e^{i \gamma_{p}}$, and a segment starting from $(\rho / 2) e^{i \gamma_{p}}$ to 0 are vanishing. Therefore, we can write the difference $v^{\mathfrak{d}_{p+1}}-v^{\mathfrak{d}_{p}}$ as a sum of three integrals:

$$
\begin{aligned}
& v^{\mathfrak{d}_{p+1}}(t, z, \epsilon)-v^{\mathfrak{d}_{p}}(t, z, \epsilon)=\frac{\kappa}{(2 \pi)^{1 / 2}} \\
& \cdot \int_{-\infty}^{+\infty} \int_{L_{\rho / 2, \gamma_{p+1}}} \omega_{\mathcal{\kappa}}^{\mathfrak{d}_{p+1}}(u, m, \epsilon) e^{-\left(u / \epsilon^{\chi+\alpha} t\right)^{\kappa}} e^{i z m} \frac{d u}{u} d m \\
& -\frac{\kappa}{(2 \pi)^{1 / 2}} \int_{-\infty}^{+\infty} \int_{L_{\rho / 2, \gamma_{p}}} \omega_{\mathcal{\kappa}}^{\mathfrak{d}_{p}}(u, m, \epsilon) \\
& \cdot e^{-\left(u / \epsilon^{\chi+\alpha} t\right)^{\kappa}} e^{i z m} \frac{d u}{u} d m+\frac{\kappa}{(2 \pi)^{1 / 2}} \\
& \cdot \int_{-\infty}^{+\infty} \int_{C_{\rho / 2, \gamma_{p}, \gamma_{p+1}}} \omega_{\kappa}(u, m, \epsilon) e^{-\left(u / \epsilon^{\chi+\alpha} t\right)^{\kappa}} e^{i z m} \frac{d u}{u} d m
\end{aligned}
$$

where $L_{\rho / 2, \gamma_{p+1}}=[\rho / 2,+\infty) e^{i \gamma_{p+1}}, L_{\rho / 2, \gamma_{p}}=[\rho / 2,+\infty) e^{i \gamma_{p}}$, and $C_{\rho / 2, \gamma_{p}, \gamma_{p+1}}$ is an arc of circle with radius connecting $(\rho / 2) e^{i \gamma_{p}}$ and $(\rho / 2) e^{i \gamma_{p+1}}$ with a well chosen orientation.
We give estimates for the quantity

$$
\begin{aligned}
I_{1} & =\mid \frac{\kappa}{(2 \pi)^{1 / 2}} \int_{-\infty}^{+\infty} \int_{L_{\rho / 2, \gamma_{p+1}}} \omega_{\kappa}^{\mathfrak{d}_{p+1}}(u, m, \epsilon) \\
& \cdot e^{-\left(u / \epsilon^{\chi+\alpha} t\right)^{\kappa}} e^{i z m} \frac{d u}{u} d m \mid .
\end{aligned}
$$

By construction, the direction $\gamma_{p+1}$ (which depends on $\epsilon^{\chi+\alpha} t$ ) is chosen in such a way that $\cos \left(\kappa\left(\gamma_{p+1}-\arg \left(\epsilon^{\chi+\alpha} t\right)\right)\right) \geq \delta_{1}$, for all $\epsilon \in \mathscr{E}_{p} \cap \mathscr{E}_{p+1}$, for all $t \in \mathscr{T}$, and for some fixed $\delta_{1}>0$. From the estimates (167), we get that

$$
\begin{aligned}
& I_{1} \leq \frac{\kappa}{(2 \pi)^{1 / 2}} \int_{-\infty}^{+\infty} \int_{\rho / 2}^{+\infty} \omega(1+|m|)^{-\mu} e^{-\beta|m|} \\
& \cdot \frac{r /|\epsilon|^{\chi+\alpha}}{1+\left(r /|\epsilon|^{\chi+\alpha}\right)^{2 \kappa}} \times \exp \left(\nu\left(\frac{r}{|\epsilon|^{\chi+\alpha}}\right)^{\kappa}\right) \\
& \cdot \exp \left(-\frac{\cos \left(\kappa\left(\gamma_{p+1}-\arg \left(\epsilon^{\chi+\alpha} t\right)\right)\right)}{\left|\epsilon^{\chi+\alpha} t\right|^{\kappa}} r^{\kappa}\right) \\
& \cdot e^{-m \operatorname{Im}(z)} \frac{d r}{r} d m \leq \frac{\kappa \omega}{(2 \pi)^{1 / 2}} \int_{-\infty}^{+\infty} e^{-\left(\beta-\beta^{\prime}\right)|m|} d m \\
& \cdot \int_{\rho / 2}^{+\infty} \frac{1}{|\epsilon|^{\chi+\alpha}} \exp \left(-\left(\frac{\delta_{1}}{|t|^{\kappa}}-\nu\right)\left(\frac{r}{|\epsilon|^{\chi+\alpha}}\right)^{\kappa}\right) d r \\
& \leq \frac{2 \kappa \omega}{(2 \pi)^{1 / 2}} \int_{0}^{+\infty} e^{-\left(\beta-\beta^{\prime}\right) m} d m \\
& \cdot \int_{\rho / 2}^{+\infty} \frac{|\epsilon|^{(\chi+\alpha)(\kappa-1)}}{\left(\delta_{1} /|t|^{\kappa}-\nu\right) \kappa(\rho / 2)^{\kappa-1}} \\
& \times \frac{\left(\delta_{1} /|t|^{\kappa}-\nu\right) \kappa r^{\kappa-1}}{|\epsilon|^{(\chi+\alpha) \kappa}} \\
& \cdot \exp \left(-\left(\frac{\delta_{1}}{|t|^{\kappa}}-\nu\right)\left(\frac{r}{|\epsilon|^{\chi+\alpha}}\right)^{\kappa}\right) d r \leq \frac{2 \kappa \omega}{(2 \pi)^{1 / 2}} \\
& \cdot \frac{|\epsilon|^{(\chi+\alpha)(\kappa-1)}}{\left(\beta-\beta^{\prime}\right)\left(\delta_{1} /|t|^{\kappa}-\nu\right) \kappa(\rho / 2)^{\kappa-1}} \\
& \cdot \exp \left(-\left(\frac{\delta_{1}}{|t|^{\kappa}}-\nu\right) \frac{(\rho / 2)^{\kappa}}{|\epsilon|^{(\chi+\alpha) \kappa}}\right) \leq \frac{2 \kappa \omega}{(2 \pi)^{1 / 2}} \\
& \cdot \frac{|\epsilon|^{(\chi+\alpha)(\kappa-1)}}{\left(\beta-\beta^{\prime}\right) \delta_{2} \kappa(\rho / 2)^{\kappa-1}} \exp \left(-\delta_{2} \frac{(\rho / 2)^{\kappa}}{|\epsilon|^{(\chi+\alpha) \kappa}}\right)
\end{aligned}
$$

for all $t \in \mathscr{T}$ and $|\operatorname{Im}(z)| \leq \beta^{\prime}$ with $|t|<\left(\delta_{1} /\left(\delta_{2}+\nu\right)\right)^{1 / \kappa}$, for some $\delta_{2}>0$, and for all $\epsilon \in \mathscr{E}_{p} \cap \mathscr{E}_{p+1}$. 
In the same way, we also give estimates for the integral

$$
\begin{aligned}
I_{2} & =\mid \frac{\kappa}{(2 \pi)^{1 / 2}} \\
& \cdot \int_{-\infty}^{+\infty} \int_{L_{\rho / 2, \gamma_{p}}} \omega_{\kappa}^{\mathfrak{d}_{p}}(u, m, \epsilon) e^{-\left(u / \epsilon^{\chi+\alpha} t\right)^{\kappa}} e^{i z m} \frac{d u}{u} d m \mid .
\end{aligned}
$$

Namely, the direction $\gamma_{p}$ (which depends on $\epsilon^{\chi+\alpha} t$ ) is chosen in such a way that $\cos \left(\kappa\left(\gamma_{p}-\arg \left(\epsilon^{\chi+\alpha} t\right)\right)\right) \geq \delta_{1}$, for all $\epsilon \epsilon$ $\mathscr{E}_{p} \cap \mathscr{E}_{p+1}$, for all $t \in \mathscr{T}$, and for some fixed $\delta_{1}>0$. Again from the estimates (167) and following the same steps as in (190), we deduce that

$$
\begin{aligned}
I_{2} \leq & \frac{2 \kappa \emptyset}{(2 \pi)^{1 / 2}} \frac{|\epsilon|^{(\chi+\alpha)(\kappa-1)}}{\left(\beta-\beta^{\prime}\right) \delta_{2} \kappa(\rho / 2)^{\kappa-1}} \\
& \cdot \exp \left(-\delta_{2} \frac{(\rho / 2)^{\kappa}}{|\epsilon|^{(\chi+\alpha) \kappa}}\right)
\end{aligned}
$$

for all $t \in \mathscr{T}$ and $|\operatorname{Im}(z)| \leq \beta^{\prime}$ with $|t|<\left(\delta_{1} /\left(\delta_{2}+\nu\right)\right)^{1 / \kappa}$, for some $\delta_{2}>0$, and for all $\epsilon \in \mathscr{E}_{p} \cap \mathscr{E}_{p+1}$.

Finally, we give upper bound estimates for the integral

$$
\begin{aligned}
I_{3} & =\mid \frac{\kappa}{(2 \pi)^{1 / 2}} \int_{-\infty}^{+\infty} \int_{C_{\rho / 2, v_{p}, v_{p+1}}} \omega_{\kappa}(u, m, \epsilon) \\
& \cdot e^{-\left(u / \epsilon^{\chi+\alpha} t\right)^{\kappa}} e^{i z m} \frac{d u}{u} d m \mid .
\end{aligned}
$$

By construction, the arc of circle $C_{\rho / 2, \gamma_{p}, \gamma_{p+1}}$ is chosen in such a way that $\cos \left(\kappa\left(\theta-\arg \left(\epsilon^{\chi+\alpha} t\right)\right)\right) \geq \delta_{1}$, for all $\theta \in\left[\gamma_{p}, \gamma_{p+1}\right]$ (if $\gamma_{p}<\gamma_{p+1}$ ) and $\theta \in\left[\gamma_{p+1}, \gamma_{p}\right]$ (if $\gamma_{p+1}<\gamma_{p}$ ), for all $t \in \mathscr{T}$, for all $\epsilon \in \mathscr{E}_{p} \cap \mathscr{E}_{p+1}$, and for some fixed $\delta_{1}>0$. Bearing in mind (167) and (118), we get that

$$
\begin{aligned}
I_{3} & \leq \frac{\kappa}{(2 \pi)^{1 / 2}} \int_{-\infty}^{+\infty} \mid \int_{\gamma_{p}}^{\gamma_{p+1}} \omega(1+|m|)^{-\mu} e^{-\beta|m|} \\
& \cdot \frac{(\rho / 2) /|\epsilon|^{\chi+\alpha}}{1+\left((\rho / 2) /|\epsilon|^{\chi+\alpha}\right)^{2 \kappa}} \times \exp \left(\nu\left(\frac{\rho / 2}{|\epsilon|^{\chi+\alpha}}\right)^{\kappa}\right) \\
\cdot & \exp \left(-\frac{\cos \left(\kappa\left(\theta-\arg \left(\epsilon^{\chi+\alpha} t\right)\right)\right)}{\left|\epsilon^{\chi+\alpha} t\right|^{\kappa}}\left(\frac{\rho}{2}\right)^{\kappa}\right) \\
& \cdot e^{-m \operatorname{Im}(z)} d \theta \mid d m \leq \frac{\kappa \omega}{(2 \pi)^{1 / 2}} \\
& \cdot \int_{-\infty}^{+\infty} e^{-\left(\beta-\beta^{\prime}\right)|m|} d m \times\left|\gamma_{p}-\gamma_{p+1}\right| \frac{\rho / 2}{|\epsilon|^{\chi+\alpha}} \\
& \cdot \exp \left(-\frac{\left(\delta_{1} /|t|^{\kappa}-\nu\right)}{2}\left(\frac{\rho / 2}{|\epsilon|^{\chi+\alpha}}\right)^{\kappa}\right) \\
& \times \exp \left(-\frac{\left(\delta_{1} /|t|^{\kappa}-\nu\right)}{2}\left(\frac{\rho / 2}{|\epsilon|^{\chi^{+\alpha}}}\right)^{\kappa}\right)
\end{aligned}
$$

$$
\begin{aligned}
& \leq \frac{2 \kappa \omega\left|\gamma_{p}-\gamma_{p+1}\right|}{(2 \pi)^{1 / 2}\left(\beta-\beta^{\prime}\right)} \sup _{x \geq 0} x^{1 / \kappa} e^{-\left(\delta_{1} /|t|^{\kappa}-\nu\right) x} \\
& \times \exp \left(-\frac{\left(\delta_{1} /|t|^{\kappa}-\nu\right)}{2}\left(\frac{\rho / 2}{|\epsilon|^{\chi+\alpha}}\right)^{\kappa}\right) \\
& \leq \frac{2 \kappa \omega\left|\gamma_{p}-\gamma_{p+1}\right|}{(2 \pi)^{1 / 2}\left(\beta-\beta^{\prime}\right)}\left(\frac{1 / \kappa}{\delta_{2}}\right)^{1 / \kappa} e^{-1 / \kappa} \\
& \cdot \exp \left(-\frac{\delta_{2}}{2}\left(\frac{\rho / 2}{|\epsilon|^{\chi^{+\alpha}}}\right)^{\kappa}\right)
\end{aligned}
$$

for all $t \in \mathscr{T}$ and $|\operatorname{Im}(z)| \leq \beta^{\prime}$ with $|t|<\left(\delta_{1} /\left(\delta_{2}+\nu\right)\right)^{1 / \kappa}$, for some $\delta_{2}>0$, and for all $\epsilon \in \mathscr{E}_{p} \cap \mathscr{E}_{p+1}$.

Finally, gathering the three above inequalities (190), (192), and (194), we deduce from decomposition (188) that

$$
\begin{aligned}
& \left|v^{\mathfrak{\delta}_{p+1}}(t, z, \epsilon)-v^{\mathfrak{\delta}_{p}}(t, z, \epsilon)\right| \leq \frac{4 \kappa \omega}{(2 \pi)^{1 / 2}} \\
& \cdot \frac{|\epsilon|^{(\chi+\alpha)(\kappa-1)}}{\left(\beta-\beta^{\prime}\right) \delta_{2} \kappa(\rho / 2)^{\kappa-1}} \exp \left(-\delta_{2} \frac{(\rho / 2)^{\kappa}}{|\epsilon|^{(\chi+\alpha) \kappa}}\right) \\
& +\frac{2 \kappa \omega\left|\gamma_{p}-\gamma_{p+1}\right|}{(2 \pi)^{1 / 2}\left(\beta-\beta^{\prime}\right)}\left(\frac{1 / \kappa}{\delta_{2}}\right)^{1 / \kappa} e^{-1 / \kappa} \\
& \cdot \exp \left(-\frac{\delta_{2}}{2}\left(\frac{\rho / 2}{|\epsilon|^{\chi+\alpha}}\right)^{\kappa}\right)
\end{aligned}
$$

for all $t \in \mathscr{T}$ and $|\operatorname{Im}(z)| \leq \beta^{\prime}$ with $|t|<\left(\delta_{1} /\left(\delta_{2}+\nu\right)\right)^{1 / k}$, for some $\delta_{2}>0$, and for all $\epsilon \in \mathscr{E}_{p} \cap \mathscr{E}_{p+1}$. Therefore, inequality (166) holds.

\section{Parametric Gevrey Asymptotic Expansions of the Solutions}

7.1. k-Summable Formal Series and Ramis-Sibuya Theorem. We recall the definition of $k$-Borel summability of formal series with coefficients in a Banach space as introduced in [5].

Definition 20. Let $k \geq 1$ be an integer. A formal series

$$
\widehat{X}(\epsilon)=\sum_{j=0}^{\infty} a_{j} \epsilon^{j} \in \mathbb{F}[[\epsilon]]
$$

with coefficients in a Banach space $\left(\mathbb{F},\|\cdot\|_{\mathbb{F}}\right)$ is said to be $k$ summable with respect to $\epsilon$ in the direction $d \in \mathbb{R}$ if

(i) the existence of $\rho \in \mathbb{R}_{+}$is ensured such that the following formal series, called formal Borel transform of $\widehat{X}$ of order $k$,

$$
\mathscr{B}_{k}(\widehat{X})(\tau)=\sum_{j=0}^{\infty} \frac{a_{j} \tau^{j}}{\Gamma(1+j / k)} \in \mathbb{F}[[\tau]]
$$

is absolutely convergent for $|\tau|<\rho$; 
(ii) one can select a $\delta>0$ such that the series $\mathscr{B}_{k}(\widehat{X})(\tau)$ can be analytically continued with respect to $\tau$ in a sector $S_{d, \delta}=\left\{\tau \in \mathbb{C}^{*}:|d-\arg (\tau)|<\delta\right\}$. Moreover, there exist $C>0$, and $K>0$, such that

$$
\left\|\mathscr{B}_{k}(\widehat{X})(\tau)\right\|_{\mathbb{F}} \leq C e^{K|\tau|^{k}}
$$

for all $\tau \in S_{d, \delta}$.

If the definition above is fulfilled, the vector valued Laplace transform of order $k$ of $\mathscr{B}_{k}(\widehat{X})(\tau)$ in the direction $d$ is set as

$$
\begin{aligned}
\mathscr{L}_{k}^{d}\left(\mathscr{B}_{k}(\widehat{X})\right)(\epsilon) \\
=\epsilon^{-k} \int_{L_{\gamma}} \mathscr{B}_{k}(\widehat{X})(u) e^{-(u / \epsilon)^{k}} k u^{k-1} d u,
\end{aligned}
$$

along a half line $L_{\gamma}=\mathbb{R}_{+} e^{i \gamma} \subset S_{d, \delta} \cup\{0\}$, where $\gamma$ depends on $\epsilon$ and is chosen in such a way that $\cos (k(\gamma-\arg (\epsilon))) \geq \delta_{1}>0$, for some fixed $\delta_{1}$, for all $\epsilon$ in a sector

$$
S_{d, \theta, R^{1 / k}}=\left\{\epsilon \in \mathbb{C}^{*}:|\epsilon|<R^{1 / k},|d-\arg (\epsilon)|<\frac{\theta}{2}\right\},
$$

where $\pi / k<\theta<\pi / k+2 \delta$ and $0<R<\delta_{1} / K$. The function $\mathscr{L}_{k}^{d}\left(\mathscr{B}_{k}(\widehat{X})\right)(\epsilon)$ is called the $k$-sum of the formal series $\widehat{X}(t)$ in the direction $d$. It is bounded and holomorphic on the sector $S_{d, \theta, R^{1 / k}}$ and has the formal series $\widehat{X}(\epsilon)$ as Gevrey asymptotic expansion of order $1 / k$ with respect to $\epsilon$ on $S_{d, \theta, R^{1 / k}}$. This means that, for all $\pi / k<\theta_{1}<\theta$, there exist $C, M>0$ such that

$$
\begin{gathered}
\left\|\mathscr{L}_{k}^{d}\left(\mathscr{B}_{k}(\widehat{X})\right)(\epsilon)-\sum_{p=0}^{n-1} a_{p} \epsilon^{p}\right\|_{\mathbb{F}} \\
\leq C M^{n} \Gamma\left(1+\frac{n}{k}\right)|\epsilon|^{n}
\end{gathered}
$$

for all $n \geq 1$, all $\epsilon \in S_{d, \theta_{1}, R^{1 / k}}$.

Now, we state a cohomological criterion for $k$ summability of formal series with coefficients in Banach spaces (see [25], p. 121 or [27], Lemma XI-2-6) which is known as the Ramis-Sibuya theorem in the literature.

Theorem (RS). Let $\left(\mathbb{F},\|\cdot\|_{\mathbb{F}}\right)$ be a Banach space over $\mathbb{C}$ and $\left\{\mathscr{E}_{p}\right\}_{0 \leq p \leq c-1}$ be a good covering in $\mathbb{C}^{*}$. For all $0 \leq p \leq \varsigma-1$, let $G_{p}$ be a holomorphic function from $\mathscr{E}_{p}$ into the Banach space $\left(\mathbb{F},\|\cdot\|_{\mathbb{F}}\right)$ and let the cocycle $\Theta_{p}(\epsilon)=G_{p+1}(\epsilon)-G_{p}(\epsilon)$ be a holomorphic function from the sector $Z_{p}=\mathscr{E}_{p+1} \cap \mathscr{E}_{p}$ into $\mathbb{F}$ (with the convention that $\mathscr{E}_{\varsigma}=\mathscr{E}_{0}$ and $G_{\zeta}=G_{0}$ ). One makes the following assumptions.

(1) The functions $G_{p}(\epsilon)$ are bounded as $\epsilon \in \mathscr{E}_{p}$ tends to the origin in $\mathbb{C}$, for all $0 \leq p \leq \varsigma-1$.

(2) The functions $\Theta_{p}(\epsilon)$ are exponentially flat of order $k$ on $Z_{p}$, for all $0 \leq p \leq \varsigma-1$. This means that there exist constants $C_{p}, A_{p}>0$ such that

$$
\left\|\Theta_{p}(\epsilon)\right\|_{\mathbb{F}} \leq C_{p} e^{-A_{p} /\left.\epsilon\right|^{k}}
$$

for all $\epsilon \in Z_{p}$, all $0 \leq p \leq \varsigma-1$.
Then, for all $0 \leq p \leq \varsigma-1$, the functions $G_{p}(\epsilon)$ are the $k$-sums on $\mathscr{E}_{p}$ of a common $k$-summable formal series $\widehat{G}(\epsilon) \epsilon$ $\mathbb{F}[[\epsilon]]$.

7.2. Parametric Gevrey Asymptotic Expansions of the Solutions and Construction of $(\chi+\alpha) \kappa$-Sums. In this subsection, we state the second main result of our work, namely, the existence of a formal power series in the parameter $\epsilon$ whose coefficients are bounded holomorphic functions on the product of a sector with small radius centered at 0 and a strip in $\mathbb{C}^{2}$ which is the common Gevrey asymptotic expansion of order $1 /(\chi+\alpha) \kappa$ of the functions $v^{\delta_{p}}(t, z, \epsilon)$ appearing in the expansion (165) of the solutions $u^{\mathfrak{d}_{p}}(t, z, \epsilon)$ to the main equations (61) and (164) established in Theorem 19.

Theorem 21. Let one assume that the hypotheses of Theorem 19 hold. Then, there exists a formal power series

$$
\widehat{v}(t, z, \epsilon)=\sum_{m \geq 0} v_{m}(t, z) \epsilon^{m}
$$

whose coefficients $v_{m}(t, z)$ belong to the Banach space $\mathbb{F}$ of bounded holomorphic functions on $(\mathscr{T} \cap D(0, \sigma)) \times H_{\beta^{\prime}}$ equipped with supremum norm, where $\sigma>0$ is defined in Theorem 19, and such that the functions $v^{\mathbb{D}_{p}}(t, z, \epsilon)$ from the decomposition (165) are its $(\chi+\alpha) \kappa$-sums on the sectors $\mathscr{E}_{p}$, for all $0 \leq p \leq \varsigma-1$, viewed as holomorphic functions from $\mathscr{E}_{p}$ into $\mathbb{F}$. In other words, for all $0 \leq p \leq \varsigma-1$, there exist two constants $C_{p}, M_{p}>0$ such that

$$
\begin{aligned}
& \sup _{t \in \mathscr{T} \cap D(0, \sigma), z \in H_{\beta^{\prime}}}\left|v^{\mathfrak{D}_{p}}(t, z, \epsilon)-\sum_{m=0}^{n-1} v_{m}(t, z) \epsilon^{m}\right| \\
& \leq C_{p} M_{p}^{n} \Gamma\left(1+\frac{n}{(\chi+\alpha) \kappa}\right)|\epsilon|^{n}
\end{aligned}
$$

for all $n \geq 1 ;$ all $\epsilon \in \mathscr{E}_{p}$.

Proof. We consider the family of functions $v^{\delta_{p}}(t, z, \epsilon), 0 \leq$ $p \leq \varsigma-1$, constructed in Theorem 19. For all $0 \leq p \leq$ $\varsigma-1$, we define $G_{p}(\epsilon):=(t, z) \mapsto v^{\mathfrak{d}_{p}}(t, z, \epsilon)$, which is by construction a holomorphic and bounded function from $\mathscr{E}_{p}$ into the Banach space $\mathbb{F}$ of bounded holomorphic functions on $(\mathscr{T} \cap D(0, \sigma)) \times H_{\beta^{\prime}}$ equipped with the supremum norm, where $\mathscr{T}$ is introduced in Definition $18, \sigma>0$ is set in Theorem 19 , and $\beta^{\prime}>0$ is the width of the strip $H_{\beta^{\prime}}$ on which the coefficients $b_{j}(z)$ are defined with respect to $z$ (see (62)). Bearing in mind the estimates (166), we see that the cocycle $\Theta_{p}(\epsilon)=G_{p+1}(\epsilon)-G_{p}(\epsilon)$ is exponentially flat of order $(\chi+\alpha) \kappa$ on $Z_{p}=\mathscr{E}_{p} \cap \mathscr{E}_{p+1}$, for any $0 \leq p \leq \varsigma-1$. Therefore, according to Theorem (RS) stated above, we obtain a formal power series

$$
\widehat{G}(\epsilon)=\sum_{m \geq 0} v_{m}(t, z) \epsilon^{m}=: \widehat{v}(t, z, \epsilon) \in \mathbb{F}[[\epsilon]]
$$

such that the functions $G_{p}(\epsilon)$ are the $(\chi+\alpha) \kappa$-sums on $\mathscr{E}_{p}$ of $\widehat{G}(\epsilon)$ as $\mathbb{F}$-valued functions, for all $0 \leq p \leq \varsigma-1$. The result follows. 


\section{Conflicts of Interest}

The author declares that there are no conflicts of interest regarding the publication of this article.

\section{References}

[1] W. Wasow, Linear turning point theory, vol. 54 of Applied Mathematical Sciences, Springer-Verlag, New York, 1985.

[2] A. Fruchard and R. Schäfke, Composite asymptotic expansions, vol. 2066 of Lecture Notes in Mathematics, Springer, Heidelberg, 2013.

[3] A. Lastra and S. Malek, "On parametric Gevrey asymptotics for some nonlinear initial value Cauchy problems," Journal of Differential Equations, vol. 259, no. 10, pp. 5220-5270, 2015.

[4] T. Mandai, "Existence and nonexistence of null-solutions for some non-Fuchsian partial differential operators with $T$ dependent coefficients," Nagoya Mathematical Journal, vol. 122, pp. 115-137, 1991.

[5] W. Balser, "Multisummable power series," in From Divergent Power Series to Analytic Functions, vol. 1582 of Lecture Notes in Mathematics, pp. 53-74, Springer Berlin Heidelberg, Berlin, Heidelberg, 1994.

[6] M. Canalis-Durand, -P. Ramis, R. Schäfke, and Y. Sibuya, "Gevrey solutions of singularly perturbed differential equations," Journal für die reine und angewandte Mathematik, vol. 518, pp. 95-129, 2000.

[7] Y. Takei, "WKB analysis and stokes geometry of differential equations," in Analytic, Algebraic and Geometric Aspects of Differential Equations, Trends in Mathematics, pp. 263-304, Springer International Publishing, Cham, 2017.

[8] O. Costin, F. Fauvet, F. Menous, and D. Sauzin, Asymptotics in Dynamics, Geometry and PDEs; Generalized Borel Summation vol. II, Edizioni della Normale, Pisa, 2011.

[9] T. M. Dunster, D. A. Lutz, and R. Schäfke, "Convergent Liouville-Green expansions for second-order linear differential equations, with an application to Bessel functions," Proceedings of the Royal Society. London. Series A. Mathematical, Physical and Engineering Sciences, vol. 440, no. 1908, pp. 37-54, 1993.

[10] O. Costin, L. Dupaigne, and M. D. Kruskal, "Borel summation of adiabatic invariants," Nonlinearity, vol. 17, no. 4, pp. 15091519, 2004.

[11] T. Koike, "On the exact WKB analysis of second order linear ordinary differential equations with simple poles," Kyoto University. Research Institute for Mathematical Sciences. Publications, vol. 36, no. 2, pp. 297-319, 2000.

[12] S. Kamimoto, T. Kawai, T. Koike, and Y. Takei, "On the WKBtheoretic structure of a Schrödinger operator with a merging pair of a simple pole and a simple turning point," Kyoto Journal of Mathematics, vol. 50, no. 1, pp. 101-164, 2010.

[13] S. Kamimoto, T. Kawai, and Y. Takei, "Exact WKB analysis of a Schrödinger equation with a merging triplet of two simple poles and one simple turning point, I-ITS WKB-theoretic transformation to the Mathieu equation," Advances in Mathematics, vol. 260, pp. 458-564, 2014.

[14] S. Kamimoto, T. Kawai, and Y. Takei, "Exact WKB analysis of a Schrödinger equation with a merging triplet of two simple poles and one simple turning point, II-Its relevance to the Mathieu equation and the Legendre equation," Advances in Mathematics, vol. 260, pp. 565-613, 2014.
[15] E. Delabaere and F. Pham, "Resurgent methods in semi-classical asymptotics," Annales de l'Institut Henri Poincaré. Physique Théorique, vol. 71, no. 1, pp. 1-94, 1999.

[16] C. Stenger, "On a conjecture of Wolfgang WASow in linear turning point theory," Comptes Rendus de l'Académie des Sciences. Série I. Mathématique, vol. 325, no. 1, pp. 27-32, 1997.

[17] N. Honda, T. Kawai, and Y. Takei, Virtual turning points, vol. 4 of SpringerBriefs in Mathematical Physics, Springer, Tokyo, 2015.

[18] T. Aoki, T. Kawai, S. Sasaki, A. Shudo, and Y. Takei, "Virtual turning points and bifurcation of Stokes curves for higher order ordinary differential equations," Journal of Physics. A. Mathematical and General, vol. 38, no. 15, pp. 3317-3336, 2005.

[19] S. Hirose, "On a WKB-theoretic transformation for a completely integrable system near a degenerate point where two turning points coalesce," Publications of the Research Institute for Mathematical Sciences, vol. 50, no. 1, pp. 19-84, 2014.

[20] B. Dubrovin, "On Hamiltonian perturbations of hyperbolic systems of conservation laws. II. Universality of critical behaviour," Communications in Mathematical Physics, vol. 267, no. 1, pp. 117139, 2006.

[21] C. Hulek, "Uniform simplification in a full neighborhood of a turning point," Comptes Rendus Mathématique. Académie des Sciences. Paris, vol. 353, no. 9, pp. 789-793, 2015.

[22] A. Lastra and S. Malek, "Multi-level Gevrey solutions of singularly perturbed linear partial differential equations," Advances in Differential Equations, vol. 21, no. 7-8, pp. 767-800, 2016.

[23] A. Lastra and S. Malek, "On parametric multisummable formal solutions to some nonlinear initial value Cauchy problems," Advances in Difference Equations, 2015:200, 78 pages, 2015.

[24] S. Malek, "On Gevrey asymptotics for some nonlinear integrodifferential equations," Journal of Dynamical and Control Systems, vol. 16, no. 3, pp. 377-406, 2010.

[25] W. Balser, Formal Power Series and Linear Systems of Meromorphic Ordinary Differential Equations, Springer, New York, NY, USA, 2000.

[26] H. Tahara and H. Yamazawa, "Multisummability of formal solutions to the Cauchy problem for some linear partial differential equations," Journal of Differential Equations, vol. 255, no. 10, pp. 3592-3637, 2013.

[27] P. Hsieh and Y. Sibuya, Basic Theory of Ordinary Differential Equations, Springer, New York, NY, USA, 1999. 


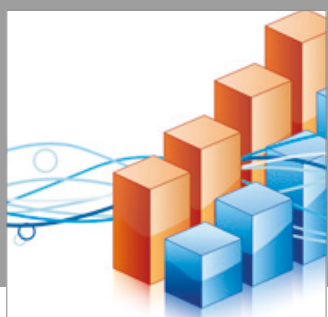

Advances in

Operations Research

vatersals

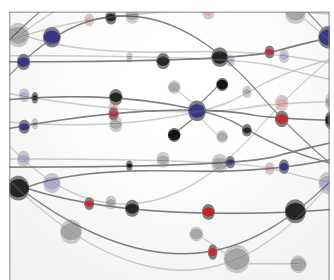

\section{The Scientific} World Journal
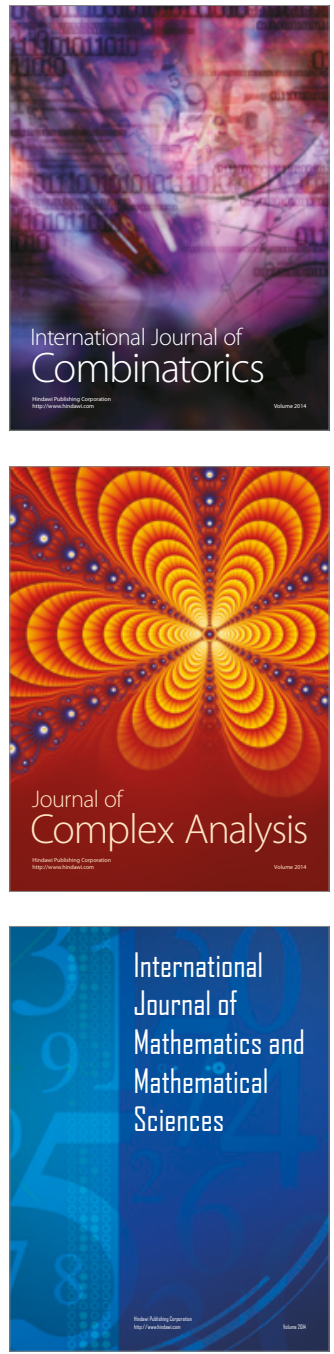
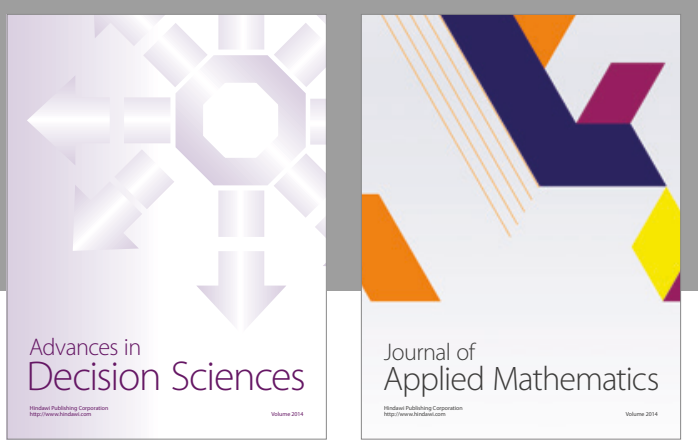

Algebra

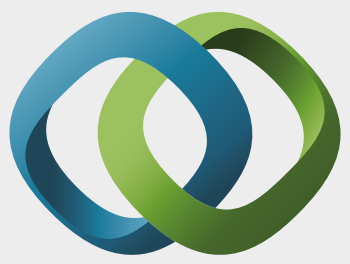

\section{Hindawi}

Submit your manuscripts at

https://www.hindawi.com
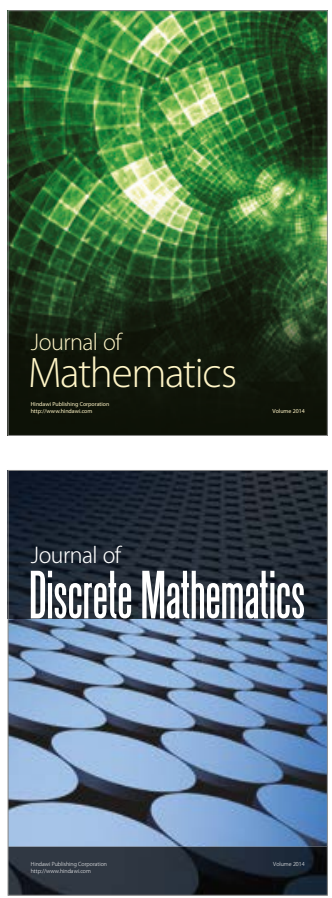

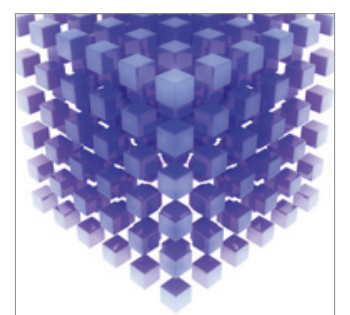

Mathematical Problems in Engineering
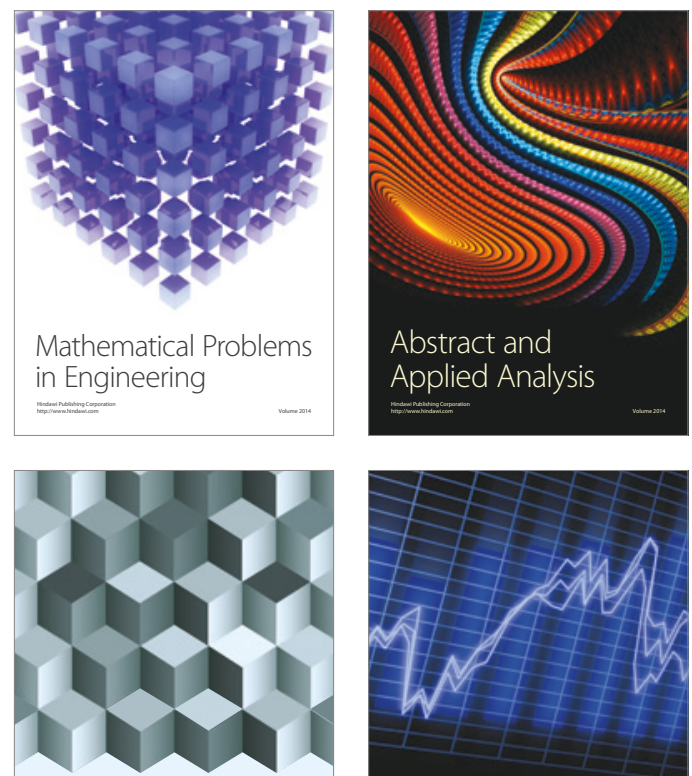

Journal of

Function Spaces

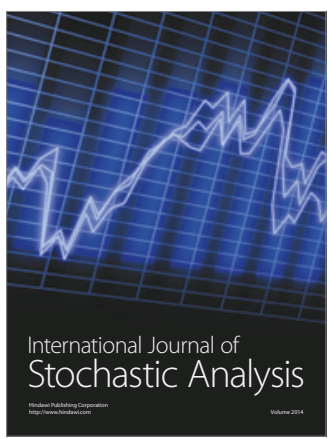

Probability and Statistics
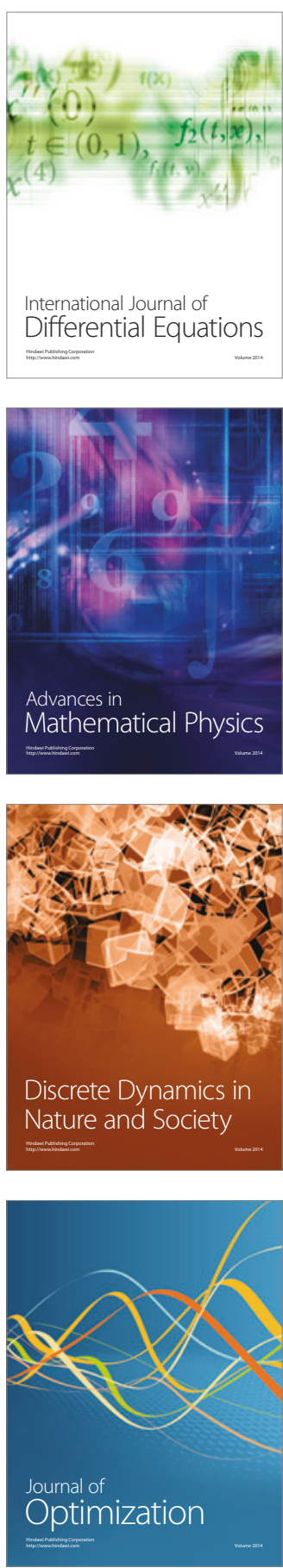